\title{
An isoperimetric inequality in the plane with a log-convex density
}

\section{McGillivray ${ }^{1}$}

Received: 20 September 2017 / Revised: 28 February 2018 / Published online: 21 March 2018 (C) The Author(s) 2018

Abstract Given a positive lower semi-continuous density $f$ on $\mathbb{R}^{2}$ the weighted volume $V_{f}:=f \mathscr{L}^{2}$ is defined on the $\mathscr{L}^{2}$-measurable sets in $\mathbb{R}^{2}$. The $f$-weighted perimeter of a set of finite perimeter $E$ in $\mathbb{R}^{2}$ is written $P_{f}(E)$. We study minimisers for the weighted isoperimetric problem

$$
I_{f}(v):=\inf \left\{P_{f}(E): E \text { is a set of finite perimeter in } \mathbb{R}^{2} \text { and } V_{f}(E)=v\right\}
$$

for $v>0$. Suppose $f$ takes the form $f: \mathbb{R}^{2} \rightarrow(0,+\infty) ; x \mapsto e^{h(|x|)}$ where $h:[0,+\infty) \rightarrow \mathbb{R}$ is a non-decreasing convex function. Let $v>0$ and $B$ a centred ball in $\mathbb{R}^{2}$ with $V_{f}(B)=v$. We show that $B$ is a minimiser for the above variational problem and obtain a uniqueness result.

Keywords Isoperimetric problem $\cdot \log$-convex density $\cdot$ Generalised mean curvature

Mathematics Subject Classification 49Q20

\section{Introduction}

Let $f$ be a positive lower semi-continuous density on $\mathbb{R}^{2}$. The weighted volume $V_{f}:=$ $f \mathscr{L}^{2}$ is defined on the $\mathscr{L}^{2}$-measurable sets in $\mathbb{R}^{2}$. Let $E$ be a set of finite perimeter in $\mathbb{R}^{2}$. The weighted perimeter of $E$ is defined by

$凶 \quad$ I. McGillivray

maiemg@bristol.ac.uk

1 School of Mathematics, University of Bristol, University Walk, Bristol BS8 1TW, UK 


$$
P_{f}(E):=\int_{\mathbb{R}^{2}} f d\left|D \chi_{E}\right| \in[0,+\infty] .
$$

We study minimisers for the weighted isoperimetric problem

$$
I_{f}(v):=\inf \left\{P_{f}(E): E \text { is a set of finite perimeter in } \mathbb{R}^{2} \text { and } V_{f}(E)=v\right\}
$$

for $v>0$. To be more specific we suppose that $f$ takes the form

$$
f: \mathbb{R}^{2} \rightarrow(0,+\infty) ; x \mapsto e^{h(|x|)}
$$

where $h:[0,+\infty) \rightarrow \mathbb{R}$ is a non-decreasing convex function. Our first main result is the following. It contains the classical isoperimetric inequality (cf. $[9,12])$ as a special case; namely, when $h$ is constant on $[0,+\infty)$.

Theorem 1.1 Let $f$ be as in (1.3) where $h:[0,+\infty) \rightarrow \mathbb{R}$ is a non-decreasing convex function. Let $v>0$ and $B$ a centred ball in $\mathbb{R}^{2}$ with $V_{f}(B)=v$. Then $B$ is a minimiser for (1.2).

For $x \geq 0$ and $v \geq 0$ define the directional derivative of $h$ in direction $v$ by

$$
h_{+}^{\prime}(x, v):=\lim _{t \downarrow 0} \frac{h(x+t v)-h(x)}{t} \in \mathbb{R}
$$

and define $h_{-}^{\prime}(x, v)$ similarly for $x>0$ and $v \leq 0$. We introduce the notation

$$
\rho_{+}:=h_{+}^{\prime}(\cdot,+1), \rho_{-}:=-h_{+}^{\prime}(\cdot,-1) \text { and } \rho:=(1 / 2)\left(\rho_{+}+\rho_{-}\right)
$$

on $(0,+\infty)$. The function $h$ is locally of bounded variation and is differentiable a.e. with $h^{\prime}=\rho$ a.e. on $(0,+\infty)$. Our second main result is a uniqueness theorem.

Theorem 1.2 Let $f$ be as in (1.3) where $h:[0,+\infty) \rightarrow \mathbb{R}$ is a non-decreasing convex function. Suppose that $R:=\inf \{\rho>0\} \in[0,+\infty)$ and set $v_{0}:=V(B(0, R))$. Let $v>0$ and $E$ a minimiser for (1.2). The following hold:

(i) if $v \leq v_{0}$ then $E$ is a.e. equivalent to a ball $B$ in $\bar{B}(0, R)$ with $V(B)=V(E)$;

(ii) if $v>v_{0}$ then $E$ is a.e. equivalent to a centred ball $B$ with $V(B)=V(E)$.

Theorem 1.1 is a generalisation of Conjecture 3.12 in [24] (due to K. Brakke) in the sense that less regularity is required of the density $f$ : in the latter, $h$ is supposed to be smooth on $(0,+\infty)$ as well as convex and non-decreasing. This conjecture springs in part from the observation that the weighted perimeter of a local volume-preserving perturbation of a centred ball is non-decreasing ([24] Theorem 3.10). In addition, the conjecture holds for log-convex Gaussian densities of the form $h:[0,+\infty) \rightarrow$ $\mathbb{R} ; t \mapsto e^{c t^{2}}$ with $c>0([3,24]$ Theorem 5.2). In subsequent work partial forms of the conjecture were proved in the literature. In [19] it is shown to hold for large $v$ provided that $h$ is uniformly convex in the sense that $h^{\prime \prime} \geq 1$ on $(0,+\infty)$ (see [19] Corollary 
6.8). A complemen tary result is contained in [11] Theorem 1.1 which establishes the conjecture for small $v$ on condition that $h^{\prime \prime}$ is locally uniformly bounded away from zero on $[0,+\infty)$. The above-mentioned conjecture is proved in large part in [7] (see Theorem 1.1) in dimension $n \geq 2$ (see also [4]). There it is assumed that the function $h$ is of class $C^{3}$ on $(0,+\infty)$ and is convex and even (meaning that $h$ is the restriction of an even function on $\mathbb{R}$ to $[0,+\infty)$ ). A uniqueness result is also obtained ( [7] Theorem 1.2). We obtain these results under weaker hypotheses in the 2-dimensional case and our proofs proceed along different lines.

We give a brief outline of the article. In Sect. 2 we discuss some preliminary material. In Sect. 3 we show that (1.2) admits an open minimiser $E$ with $C^{1}$ boundary $M$ (Theorem 3.8). The argument draws upon the regularity theory for almost minimal sets (cf. [27]) and includes an adaptation of [21] Proposition 3.1. In Sect. 4 it is shown that the boundary $M$ is of class $C^{1,1}$ (and has weakly bounded curvature). This result is contained in [21] Corollary 3.7 (see also [8]) but we include a proof for completeness. This Section also includes the result that $E$ may be supposed to possess spherical cap symmetry (Theorem 4.5). Section 5 contains further results on spherical cap symmetric sets useful in the sequel. The main result of Sect. 6 is Theorem 6.5 which shows that the generalised (mean) curvature is conserved along $M$ in a weak sense. In Sect. 7 it is shown that there exist convex minimisers of (1.2). Sections 8 and 9 comprise an analytic interlude and are devoted to the study of solutions of the firstorder differential equation that appears in Theorem 6.6 subject to Dirichlet boundary conditions. Section 9 for example contains a comparison theorem for solutions to a Ricatti equation (Theorem 9.15 and Corollary 9.16). These are new as far as the author is aware. Section 10 concludes the proof of our main theorems.

\section{Some preliminaries}

Geometric measure theory. We use $|\cdot|$ to signify the Lebesgue measure on $\mathbb{R}^{2}$ (or occasionally $\mathscr{L}^{2}$ ). Let $E$ be a $\mathscr{L}^{2}$-measurable set in $\mathbb{R}^{2}$. The set of points in $E$ with density $t \in[0,1]$ is given by

$$
E^{t}:=\left\{x \in \mathbb{R}^{2}: \lim _{\rho \downarrow 0} \frac{|E \cap B(x, \rho)|}{|B(x, \rho)|}=t\right\} .
$$

As usual $B(x, \rho)$ denotes the open ball in $\mathbb{R}^{2}$ with centre $x \in \mathbb{R}^{2}$ and radius $\rho>0$. The set $E^{1}$ is the measure-theoretic interior of $E$ while $E^{0}$ is the measure-theoretic exterior of $E$. The essential boundary of $E$ is the set $\partial^{\star} E:=\mathbb{R}^{2} \backslash\left(E^{0} \cup E^{1}\right)$.

Recall that an integrable function $u$ on $\mathbb{R}^{2}$ is said to have bounded variation if the distributional derivative of $u$ is representable by a finite Radon measure $D u$ (cf. [1] Definition 3.1 for example) with total variation $|D u|$; in this case, we write $u \in$ $\mathrm{BV}\left(\mathbb{R}^{2}\right)$. The set $E$ has finite perimeter if $\chi_{E}$ belongs to $\mathrm{BV}_{\text {loc }}\left(\mathbb{R}^{2}\right)$. The reduced boundary $\mathscr{F} E$ of $E$ is defined by

$$
\begin{gathered}
\mathscr{F} E:=\left\{x \in \operatorname{supp}\left|D \chi_{E}\right|: v^{E}(x):=\lim _{\rho \downarrow 0} \frac{D \chi_{E}(B(x, \rho))}{\left|D \chi_{E}\right|(B(x, \rho))}\right. \\
\text { exists in } \left.\mathbb{R}^{2} \text { and }\left|v^{E}(x)\right|=1\right\}
\end{gathered}
$$


(cf. [1] Definition 3.54) and is a Borel set (cf. [1] Theorem 2.22 for example). We use $\mathscr{H}^{k}(k \in[0,+\infty))$ to stand for $k$-dimensional Hausdorff measure. If $E$ is a set of finite perimeter in $\mathbb{R}^{2}$ then

$$
\mathscr{F} E \subset E^{1 / 2} \subset \partial^{*} E \text { and } \mathscr{H}^{1}\left(\partial^{*} E \backslash \mathscr{F} E\right)=0
$$

by [1] Theorem 3.61.

Let $f$ be a positive locally Lipschitz density on $\mathbb{R}^{2}$. Let $E$ be a set of finite perimeter and $U$ a bounded open set in $\mathbb{R}^{2}$. The weighted perimeter of $E$ relative to $U$ is defined by

$$
P_{f}(E, U):=\sup \left\{\int_{U} \operatorname{div}(f X) d x: X \in C_{c}^{\infty}\left(U, \mathbb{R}^{2}\right),\|X\|_{\infty} \leq 1\right\}
$$

By the Gauss-Green formula ( [1] Theorem 3.36 for example) and a convolution argument,

$$
\begin{aligned}
P_{f}(E, U)= & \sup \left\{\int_{\mathbb{R}^{2}} f\left\langle v^{E}, X\right\rangle d\left|D \chi_{E}\right|: X \in C_{c}^{\infty}\left(\mathbb{R}^{2}, \mathbb{R}^{2}\right),\right. \\
& \left.\quad \operatorname{supp}[X] \subset U,\|X\|_{\infty} \leq 1\right\} \\
= & \sup \left\{\int_{\mathbb{R}^{2}} f\left\langle v^{E}, X\right\rangle d\left|D \chi_{E}\right|: X \in C_{c}\left(\mathbb{R}^{2}, \mathbb{R}^{2}\right),\right. \\
& \left.\operatorname{supp}[X] \subset U,\|X\|_{\infty} \leq 1\right\} \\
= & \int_{U} f d\left|D \chi_{E}\right|
\end{aligned}
$$

where we have also used [1] Propositions 1.47 and 1.23.

Lemma 2.1 Let $\varphi$ be a $C^{1}$ diffeomeorphism of $\mathbb{R}^{2}$ which coincides with the identity map on the complement of a compact set and $E \subset \mathbb{R}^{2}$ with $\chi_{E} \in \mathrm{BV}\left(\mathbb{R}^{2}\right)$. Then

(i) $\chi_{\varphi(E)} \in \mathrm{BV}\left(\mathbb{R}^{2}\right)$;

(ii) $\partial^{\star} \varphi(E)=\varphi\left(\partial^{\star} E\right)$;

(iii) $\mathscr{H}^{1}(\mathscr{F} \varphi(E) \Delta \varphi(\mathscr{F} E))=0$.

Proof Part ( $i$ ) follows from [1] Theorem 3.16 as $\varphi$ is a proper Lipschitz function. Given $x \in E^{0}$ we claim that $y:=\varphi(x) \in \varphi(E)^{0}$. Let $M$ stand for the Lipschitz constant of $\varphi$ and $L$ stand for the Lipschitz constant of $\varphi^{-1}$. Note that $B(y, r) \subset \varphi(B(x, L r))$ for each $r>0$. As $\varphi$ is a bijection and using [1] Proposition 2.49,

$$
\begin{aligned}
|\varphi(E) \cap B(y, r)| & \leq \mid \varphi(E) \cap \varphi(B(x, L r) \mid \\
& =|\varphi(E \cap B(x, L r))| \leq M^{2}|E \cap B(x, L r)| .
\end{aligned}
$$


This means that

$$
\frac{|\varphi(E) \cap B(y, r)|}{|B(y, r)|} \leq(L M)^{2} \frac{|E \cap B(x, L r)|}{|B(x, L r)|}
$$

for $r>0$ and this proves the claim. This entails that $\varphi\left(E^{0}\right) \subset[\varphi(E)]^{0}$. The reverse inclusion can be seen using the fact that $\varphi$ is a bijection. In summary $\varphi\left(E^{0}\right)=[\varphi(E)]^{0}$. The corresponding identity for $E^{1}$ can be seen in a similar way. These identities entail (ii). From (2.1) and (ii) we may write $\mathscr{F} \varphi(E) \cup N_{1}=\varphi(\mathscr{F} E) \cup \varphi\left(N_{2}\right)$ for $\mathscr{H}^{1}$-null sets $N_{1}, N_{2}$ in $\mathbb{R}^{2}$. Item (iii) follows.

Curves with weakly bounded curvature. Suppose the open set $E$ in $\mathbb{R}^{2}$ has $C^{1}$ boundary $M$. Denote by $n: M \rightarrow \mathbb{S}^{1}$ the inner unit normal vector field. Given $p \in M$ we choose a tangent vector $t(p) \in \mathbb{S}^{1}$ in such a way that the pair $\{t(p), n(p)\}$ forms a positively oriented basis for $\mathbb{R}^{2}$. There exists a local parametrisation $\gamma_{1}: I \rightarrow M$ where $I=(-\delta, \delta)$ for some $\delta>0$ of class $C^{1}$ with $\gamma_{1}(0)=p$. We always assume that $\gamma_{1}$ is parametrised by arc-length and that $\dot{\gamma}_{1}(0)=t(p)$ where the dot signifies differentiation with respect to arc-length. Let $X$ be a vector field defined in some neighbourhood of $p$ in $M$. Then

$$
\left(D_{t} X\right)(p):=\left.\frac{d}{d s}\right|_{s=0}\left(X \circ \gamma_{1}\right)(s)
$$

if this limit exists and the divergence $\operatorname{div}^{M} X$ of $X$ along $M$ at $p$ is defined by

$$
\operatorname{div}^{M} X:=\left\langle D_{t} X, t\right\rangle
$$

evaluated at $p$. Suppose that $X$ is a vector field in $C^{1}\left(U, \mathbb{R}^{2}\right)$ where $U$ is an open neighbourhood of $p$ in $\mathbb{R}^{2}$. Then

$$
\operatorname{div} X=\operatorname{div}^{M} X+\left\langle D_{n} X, n\right\rangle
$$

at $p$. If $p \in M \backslash\{0\}$ let $\sigma(p)$ stand for the angle measured anti-clockwise from the position vector $p$ to the tangent vector $t(p) ; \sigma(p)$ is uniquely determined up to integer multiples of $2 \pi$.

Let $E$ be an open set in $\mathbb{R}^{2}$ with $C^{1,1}$ boundary $M$. Let $x \in M$ and $\gamma_{1}: I \rightarrow M$ a local parametrisation of $M$ in a neighbourhood of $x$. There exists a constant $c>0$ such that

$$
\left|\dot{\gamma}_{1}\left(s_{2}\right)-\dot{\gamma}_{1}\left(s_{1}\right)\right| \leq c\left|s_{2}-s_{1}\right|
$$

for $s_{1}, s_{2} \in I$; a constraint on average curvature (cf. [10,18]). That is, $\dot{\gamma}_{1}$ is Lipschitz on $I$. So $\dot{\gamma}_{1}$ is absolutely continuous and differentiable a.e. on $I$ with

$$
\dot{\gamma}_{1}\left(s_{2}\right)-\dot{\gamma}_{1}\left(s_{1}\right)=\int_{s_{1}}^{s_{2}} \ddot{\gamma}_{1} d s
$$


for any $s_{1}, s_{2} \in I$ with $s_{1}<s_{2}$. Moreover, $\left|\ddot{\gamma}_{1}\right| \leq c$ a.e. on $I$ (cf. [1] Corollary 2.23). As $\left\langle\dot{\gamma}_{1}, \dot{\gamma}_{1}\right\rangle=1$ on $I$ we see that $\left\langle\dot{\gamma}_{1}, \ddot{\gamma}_{1}\right\rangle=0$ a.e. on $I$. The (geodesic) curvature $k_{1}$ is then defined a.e. on $I$ via the relation

$$
\ddot{\gamma}_{1}=k_{1} n_{1}
$$

as in [18]. The curvature $k$ of $M$ is defined $\mathscr{H}^{1}$-a.e. on $M$ by

$$
k(x):=k_{1}(s)
$$

whenever $x=\gamma_{1}(s)$ for some $s \in I$ and $k_{1}(s)$ exists. We sometimes write $H(\cdot, E)=k$.

Let $E$ be an open set in $\mathbb{R}^{2}$ with $C^{1}$ boundary $M$. Let $x \in M$ and $\gamma_{1}: I \rightarrow M$ a local parametrisation of $M$ in a neighbourhood of $x$. In case $\gamma_{1} \neq 0$ let $\theta_{1}$ stand for the angle measured anti-clockwise from $e_{1}$ to the position vector $\gamma_{1}$ and $\sigma_{1}$ stand for the angle measured anti-clockwise from the position vector $\gamma_{1}$ to the tangent vector $t_{1}=\dot{\gamma}_{1}$. Put $r_{1}:=\left|\gamma_{1}\right|$ on $I$. Then $r_{1}, \theta_{1} \in C^{1}(I)$ and

$$
\begin{array}{r}
\dot{r}_{1}=\cos \sigma_{1} ; \\
r_{1} \dot{\theta}_{1}=\sin \sigma_{1} ;
\end{array}
$$

on $I$ provided that $\gamma_{1} \neq 0$. Now suppose that $M$ is of class $C^{1,1}$. Let $\alpha_{1}$ stand for the angle measured anti-clockwise from the fixed vector $e_{1}$ to the tangent vector $t_{1}$ (uniquely determined up to integer multiples of $2 \pi$ ). Then $t_{1}=\left(\cos \alpha_{1}, \sin \alpha_{1}\right)$ on $I$ so $\alpha_{1}$ is absolutely continuous on $I$. In particular, $\alpha_{1}$ is differentiable a.e. on $I$ with $\dot{\alpha}_{1}=k_{1}$ a.e. on $I$. This means that $\alpha_{1} \in C^{0,1}(I)$. In virtue of the identities $r_{1} \cos \sigma_{1}=\left\langle\gamma_{1}, t_{1}\right\rangle$ and $r_{1} \sin \sigma_{1}=-\left\langle\gamma_{1}, n_{1}\right\rangle$ we see that $\sigma_{1}$ is absolutely continuous on $I$ and $\sigma_{1} \in C^{0,1}(I)$. By choosing an appropriate branch we may assume that

$$
\alpha_{1}=\theta_{1}+\sigma_{1}
$$

on $I$. We may choose $\sigma$ in such a way that $\sigma \circ \gamma_{1}=\sigma_{1}$ on $I$.

Flows. Recall that a diffeomorphism $\varphi: \mathbb{R}^{2} \rightarrow \mathbb{R}^{2}$ is said to be proper if $\varphi^{-1}(K)$ is compact whenever $K \subset \mathbb{R}^{2}$ is compact. Given $X \in C_{c}^{\infty}\left(\mathbb{R}^{2}, \mathbb{R}^{2}\right)$ there exists a 1parameter group of proper $C^{\infty}$ diffeomorphisms $\varphi: \mathbb{R} \times \mathbb{R}^{2} \rightarrow \mathbb{R}^{2}$ as in [20] Lemma 2.99 that satisfy

$$
\begin{aligned}
\partial_{t} \varphi(t, x) & =X(\varphi(t, x)) \text { for each }(t, x) \in \mathbb{R} \times \mathbb{R}^{2} \\
\varphi(0, x) & =x \text { for each } x \in \mathbb{R}^{2} .
\end{aligned}
$$

We often use $\varphi_{t}$ to refer to the diffeomorphism $\varphi(t, \cdot): \mathbb{R}^{2} \rightarrow \mathbb{R}^{2}$.

Lemma 2.2 Let $X \in C_{c}^{\infty}\left(\mathbb{R}^{2}, \mathbb{R}^{2}\right)$ and $\varphi$ be the corresponding flow as above. Then

(i) there exists $R \in C^{\infty}\left(\mathbb{R} \times \mathbb{R}^{2}, \mathbb{R}^{2}\right)$ and $K>0$ such that

$$
\varphi(t, x)= \begin{cases}x+t X(x)+R(t, x) & \text { for } x \in \operatorname{supp}[X] \\ x & \text { for } x \notin \operatorname{supp}[X]\end{cases}
$$


where $|R(t, x)| \leq K t^{2}$ for $(t, x) \in \mathbb{R} \times \mathbb{R}^{2}$;

(ii) there exists $R^{(1)} \in C^{\infty}\left(\mathbb{R} \times \mathbb{R}^{2}, M_{2}(\mathbb{R})\right)$ and $K_{1}>0$ such that

$$
d \varphi(t, x)= \begin{cases}I+t d X(x)+R^{(1)}(t, x) & \text { for } x \in \operatorname{supp}[X] \\ I & \text { for } x \notin \operatorname{supp}[X]\end{cases}
$$

where $\left|R^{(1)}(t, x)\right| \leq K_{1} t^{2}$ for $(t, x) \in \mathbb{R} \times \mathbb{R}^{2}$;

(iii) there exists $R^{(2)} \in C^{\infty}\left(\mathbb{R} \times \mathbb{R}^{2}, \mathbb{R}\right)$ and $K_{2}>0$ such that

$$
J_{2} d \varphi(t, x)= \begin{cases}1+t \operatorname{div} X(x)+R^{(2)}(t, x) & \text { for } x \in \operatorname{supp}[X] ; \\ 1 & \text { for } x \notin \operatorname{supp}[X]\end{cases}
$$

where $\left|R^{(2)}(t, x)\right| \leq K_{2} t^{2}$ for $(t, x) \in \mathbb{R} \times \mathbb{R}^{2}$.

Let $x \in \mathbb{R}^{2}$, $v$ a unit vector in $\mathbb{R}^{2}$ and $M$ the line though $x$ perpendicular to $v$. Then (iv) there exists $R^{(3)} \in C^{\infty}\left(\mathbb{R} \times \mathbb{R}^{2}, \mathbb{R}\right)$ and $K_{3}>0$ such that

$$
J_{1} d^{M} \varphi(t, x)= \begin{cases}1+t\left(\operatorname{div}^{M} X\right)(x)+R^{(3)}(t, x) & \text { for } x \in \operatorname{supp}[X] \\ 1 & \text { for } x \notin \operatorname{supp}[X]\end{cases}
$$

where $\left|R^{(3)}(t, x)\right| \leq K_{3} t^{2}$ for $(t, x) \in \mathbb{R} \times \mathbb{R}^{2}$.

Proof (i) First notice that $\varphi \in C^{\infty}\left(\mathbb{R} \times \mathbb{R}^{2}\right)$ by [16] Theorem 3.3 and Exercise 3.4. The statement for $x \notin \operatorname{supp}[X]$ follows by uniqueness (cf. [16] Theorem 3.1); the assertion for $x \in \operatorname{supp}[X]$ follows from Taylor's theorem. (ii) follows likewise: note, for example, that

$$
\left.\left[\partial_{t t} d \varphi\right]_{\alpha \beta}\right|_{t=0}=X_{, \beta \delta}^{\alpha} X^{\delta}+X_{, \gamma}^{\alpha} X_{, \beta}^{\gamma}
$$

where the subscript, signifies partial differentiation. (iii) follows from (ii) and the definition of the 2-dimensional Jacobian (cf. [1] Definition 2.68). (iv) Using [1] Definition 2.68 together with the Cauchy-Binet formula [1] Proposition 2.69, $J_{1} d^{M} \varphi(t, x)=|d \varphi(t, x) v|$ for $t \in \mathbb{R}$ and the result follows from $(i i)$.

Let $I$ be an open interval in $\mathbb{R}$ containing 0 . Let $Z: I \times \mathbb{R}^{2} \rightarrow \mathbb{R}^{2} ;(t, x) \mapsto Z(t, x)$ be a continuous time-dependent vector field on $\mathbb{R}^{2}$ with the properties

(Z.1) $Z(t, \cdot) \in C_{c}^{1}\left(\mathbb{R}^{2}, \mathbb{R}^{2}\right)$ for each $t \in I$;

(Z.2) $\operatorname{supp}[Z(t, \cdot)] \subset K$ for each $t \in I$ for some compact set $K \subset \mathbb{R}^{2}$.

By [16] Theorems I.1.1, I.2.1, I.3.1, I.3.3 there exists a unique flow $\varphi: I \times \mathbb{R}^{2} \rightarrow \mathbb{R}^{2}$ such that

(F.1) $\varphi: I \times \mathbb{R}^{2} \rightarrow \mathbb{R}^{2}$ is of class $C^{1}$;

(F.2) $\varphi(0, x)=x$ for each $x \in \mathbb{R}^{2}$;

(F.3) $\partial_{t} \varphi(t, x)=Z(t, \varphi(x, t))$ for each $(t, x) \in I \times \mathbb{R}^{2}$;

(F.4) $\varphi_{t}:=\varphi(t, \cdot): \mathbb{R}^{2} \rightarrow \mathbb{R}^{2}$ is a proper diffeomorphism for each $t \in I$. 
Lemma 2.3 Let $Z$ be a time-dependent vector field with the properties $(Z .1)-(Z .2)$ and $\varphi$ be the corresponding flow. Then

(i) for $(t, x) \in I \times \mathbb{R}^{2}$,

$$
d \varphi(t, x)= \begin{cases}I+t d Z_{0}(x)+t R(t, x) & \text { for } x \in K \\ I & \text { for } x \notin K\end{cases}
$$

where $\sup _{K}|R(t, \cdot)| \rightarrow 0$ as $t \rightarrow 0$.

Let $x \in \mathbb{R}^{2}$, $v$ a unit vector in $\mathbb{R}^{2}$ and $M$ the line though $x$ perpendicular to $v$. Then (ii) for $(t, x) \in I \times \mathbb{R}^{2}$,

$$
J_{1} d^{M} \varphi(t, x)= \begin{cases}1+t\left(\operatorname{div}^{M} Z_{0}\right)(x)+t R^{(1)}(t, x) & \text { for } x \in K \\ 1 & \text { for } x \notin K\end{cases}
$$

where $\sup _{K}\left|R^{(1)}(t, \cdot)\right| \rightarrow 0$ as $t \rightarrow 0$.

Proof (i) We first remark that the flow $\varphi: I \times \mathbb{R}^{2} \rightarrow \mathbb{R}^{2}$ associated to $Z$ is continuously differentiable in $t, x$ in virtue of (Z.1) by [16] Theorem I.3.3. Put $y(t, x):=d \varphi(t, x)$ for $(t, x) \in I \times \mathbb{R}^{2}$. By [16] Theorem I.3.3,

$$
\dot{y}(t, x)=d Z(t, \varphi(t, x)) y(t, x)
$$

for each $(t, x) \in I \times \mathbb{R}^{2}$ and $y(0, x)=I$ for each $x \in \mathbb{R}^{2}$ where $I$ stands for the $2 \times 2$-identity matrix. For $x \in K$ and $t \in I$,

$$
\begin{aligned}
d \varphi(t, x) & =I+d \varphi(t, x)-d \varphi(0, x) \\
& =I+t \dot{y}(0, x)+t\left\{\frac{d \varphi(t, x)-d \varphi(0, x)}{t}-\dot{y}(0, x)\right\} \\
& =I+t d Z(0, x)+t\left\{\frac{y(t, x)-y(0, x)}{t}-\dot{y}(0, x)\right\} \\
& =I+t d Z_{0}(x)+t\left\{\frac{y(t, x)-y(0, x)}{t}-\dot{y}(0, x)\right\} .
\end{aligned}
$$

Applying the mean-value theorem component-wise and using uniform continuity of the matrix $\dot{y}$ in its arguments we see that

$$
\frac{y(t, \cdot)-y(0, \cdot)}{t}-\dot{y}(0, \cdot) \rightarrow 0
$$

uniformly on $K$ as $t \rightarrow 0$. This leads to (i). Part (ii) follows as in Lemma 2.2.

Let $E$ be a set of finite perimeter in $\mathbb{R}^{2}$ with $V_{f}(E)<+\infty$. The first variation of weighted volume resp. perimeter along $X \in C_{c}^{\infty}\left(\mathbb{R}^{2}, \mathbb{R}^{2}\right)$ is defined by

$$
\delta V_{f}(X):=\left.\frac{d}{d t}\right|_{t=0} V_{f}\left(\varphi_{t}(E)\right),
$$




$$
\delta P_{f}^{+}(X):=\lim _{t \downarrow 0} \frac{P_{f}\left(\varphi_{t}(E)\right)-P_{f}(E)}{t},
$$

whenever the limit exists. By Lemma 2.1 the $f$-perimeter in (2.14) is well-defined. Convex functions. Suppose that $h:[0,+\infty) \rightarrow \mathbb{R}$ is a convex function. For $x \geq 0$ and $v \geq 0$ define

$$
h_{+}^{\prime}(x, v):=\lim _{t \downarrow 0} \frac{h(x+t v)-h(x)}{t} \in \mathbb{R}
$$

and define $h_{-}^{\prime}(x, v)$ similarly for $x>0$ and $v \leq 0$. For future use we introduce the notation

$$
\rho_{+}:=h^{\prime}(\cdot,+1), \rho_{-}:=-h^{\prime}(\cdot,-1) \text { and } \rho:=(1 / 2)\left(\rho_{+}+\rho_{-}\right)
$$

on $(0,+\infty)$. It holds that $h$ is differentiable a.e. and $h^{\prime}=\rho$ a.e. on $(0,+\infty)$. Define $[\rho]:=\rho_{+}-\rho_{-}$. Then $[\rho] \geq 0$ and vanishes a.e. on $(0,+\infty)$.

Lemma 2.4 Suppose that the function $f$ takes the form (1.3) where $h:[0,+\infty) \rightarrow \mathbb{R}$ is a convex function. Then

(i) the directional derivative $f_{+}^{\prime}(x, v)$ exists in $\mathbb{R}$ for each $x \in \mathbb{R}^{2}$ and $v \in \mathbb{R}^{2}$;

(ii) for $v \in \mathbb{R}^{2}$,

$$
f_{+}^{\prime}(x, v)= \begin{cases}f(x) h_{+}^{\prime}(|x|, \operatorname{sgn}\langle x, v\rangle) \frac{|\langle x, v\rangle|}{|x|} & \text { for } x \in \mathbb{R}^{2} \backslash\{0\} \\ f(0) h_{+}^{\prime}(0,+1)|v| & \text { for } x=0\end{cases}
$$

(iii) if $M$ is a $C^{1}$ hypersurface in $\mathbb{R}^{2}$ such that $\cos \sigma \neq 0$ on $M$ then $f$ is differentiable $\mathscr{H}^{1}$-a.e. on $M$ and

$$
(\nabla f)(x)=f(x) \rho(|x|) \frac{\langle x, \cdot\rangle}{|x|}
$$

for $\mathscr{H}^{1}$-a.e. $x \in M$.

Proof The assertion in (i) follows from the monotonicity of chords property while (ii) is straightforward. (iii) Let $x \in M$ and $\gamma_{1}: I \rightarrow M$ be a $C^{1}$-parametrisation of $M$ near $x$ as above. Now $r_{1} \in C^{1}(I)$ and $\dot{r_{1}}(0)=\cos \sigma(x) \neq 0$ so we may assume that $r_{1}: I \rightarrow r_{1}(I) \subset(0,+\infty)$ is a $C^{1}$ diffeomorphism. The differentiability set $D(h)$ of $h$ has full Lebesgue measure in [0, $+\infty)$. It follows by [1] Proposition 2.49 that $r_{1}^{-1}(D(h))$ has full measure in $I$. This entails that $f$ is differentiable $\mathscr{H}^{1}$-a.e. on $\gamma_{1}(I) \subset M$.

\section{Existence and $C^{1}$ regularity}

We start with an existence theorem. 
Theorem 3.1 Assume that $f$ is a positive radial lower-semicontinuous non-decreasing density on $\mathbb{R}^{2}$ which diverges to infinity. Then for each $v>0$,

(i) (1.2) admits a minimiser;

(ii) any minimiser of (1.2) is essentially bounded.

Proof See [22] Theorems 3.3 and 5.9.

But the bulk of this section will be devoted to a discussion of $C^{1}$ regularity.

Proposition 3.2 Let $f$ be a positive locally Lipschitz density on $\mathbb{R}^{2}$. Let $E \subset \mathbb{R}^{2}$ be a bounded set with finite perimeter. Let $X \in C_{c}^{\infty}\left(\mathbb{R}^{2}, \mathbb{R}^{2}\right)$. Then

$$
\delta V_{f}(X)=\int_{E} \operatorname{div}(f X) d x=-\int_{\mathscr{F} E} f\left\langle v^{E}, X\right\rangle d \mathscr{H}^{1}
$$

Proof Let $t \in \mathbb{R}$. By the area formula ([1] Theorem 2.71 and (2.74)),

$$
V_{f}\left(\varphi_{t}(E)\right)=\int_{\varphi_{t}(E)} f d x=\int_{E}\left(f \circ \varphi_{t}\right) J_{2} d\left(\varphi_{t}\right)_{x} d x
$$

and

$$
\begin{aligned}
V_{f}\left(\varphi_{t}(E)\right)-V_{f}(E) & =\int_{E}\left(f \circ \varphi_{t}\right) J_{2} d \varphi_{t}-f d x \\
& =\int_{E}\left(f \circ \varphi_{t}\right)\left(J_{2} d \varphi_{t}-1\right) d x+\int_{E} f \circ \varphi_{t}-f d x
\end{aligned}
$$

The density $f$ is locally Lipschitz and in particular differentiable a.e. on $\mathbb{R}^{2}$ (see [1] 2.3 for example). By the dominated convergence theorem and Lemma 2.2,

$$
\begin{aligned}
\delta V_{f}(X) & =\int_{E}\{f \operatorname{div}(X)+\langle\nabla f, X\rangle\} d x=\int_{E} \operatorname{div}(f X) d x \\
& =-\int_{\mathscr{F} E} f\left\langle v^{E}, X\right\rangle d \mathscr{H}^{1}
\end{aligned}
$$

by the generalised Gauss-Green formula [1] Theorem 3.36.

Proposition 3.3 Let $f$ be a positive locally Lipschitz density on $\mathbb{R}^{2}$. Let $E \subset \mathbb{R}^{2}$ be a bounded set with finite perimeter. Let $X \in C_{c}^{\infty}\left(\mathbb{R}^{2}, \mathbb{R}^{2}\right)$. Then there exist constants $C>0$ and $\delta>0$ such that

$$
\left|P_{f}\left(\varphi_{t}(E)\right)-P_{f}(E)\right| \leq C|t|
$$

for $|t|<\delta$. 
Proof Let $t \in \mathbb{R}$. By Lemma 2.1 and [1] Theorem 3.59,

$$
P_{f}\left(\varphi_{t}(E)\right)=\int_{\mathbb{R}^{2}} f d\left|D \chi_{\varphi_{t}(E)}\right|=\int_{\mathscr{F} \varphi_{t}(E)} f d \mathscr{H}^{1}=\int_{\varphi_{t}(\mathscr{F} E)} f d \mathscr{H}^{1} .
$$

As $\mathscr{F} E$ is countably 1-rectifiable ( [1] Theorem 3.59) we may use the generalised area formula [1] Theorem 2.91 to write

$$
P_{f}\left(\varphi_{t}(E)\right)=\int_{\mathscr{F} E}\left(f \circ \varphi_{t}\right) J_{1} d^{\mathscr{F} E}\left(\varphi_{t}\right)_{x} d \mathscr{H}^{1}
$$

For each $x \in \mathscr{F} E$ and any $t \in \mathbb{R}$,

$$
\left|\left(f \circ \varphi_{t}\right)(x)-f(x)\right| \leq K|\varphi(t, x)-x| \leq K\|X\|_{\infty}|t|
$$

where $K$ is the Lipschitz constant of $f$ on $\operatorname{supp}[X]$. The result follows upon writing

$$
\begin{aligned}
P_{f}\left(\varphi_{t}(E)\right)-P_{f}(E)= & \int_{\mathscr{F} E}\left(f \circ \varphi_{t}\right)\left(J_{1} d^{\mathscr{F} E}\left(\varphi_{t}\right)_{x}-1\right) \\
& +\left[f \circ \varphi_{t}-f\right] d \mathscr{H}^{1}
\end{aligned}
$$

and using Lemma 2.2.

Lemma 3.4 Let $f$ be a positive locally Lipschitz density on $\mathbb{R}^{2}$. Let $E \subset \mathbb{R}^{2}$ be a bounded set with finite perimeter and $p \in \mathscr{F} E$. For any $r>0$ there exists $X \in$ $C_{c}^{\infty}\left(\mathbb{R}^{2}, \mathbb{R}^{2}\right)$ with $\operatorname{supp}[X] \subset B(p, r)$ such that $\delta V_{f}(X)=1$.

Proof By (2.2) and [1] Theorem 3.59 and (3.57) in particular,

$$
P_{f}(E, B(p, r))=\int_{B(p, r) \cap \mathscr{F} E} f d \mathscr{H}^{1}>0
$$

for any $r>0$. By the variational characterisation of the $f$-perimeter relative to $B(p, r)$ we can find $Y \in C_{c}^{\infty}\left(\mathbb{R}^{2}, \mathbb{R}^{2}\right)$ with $\operatorname{supp}[Y] \subset B(p, r)$ such that

$$
0<\int_{E \cap B(p, r)} \operatorname{div}(f Y) d x=-\int_{\mathscr{F} E \cap B(p, r)} f\left\langle v^{E}, Y\right\rangle d \mathscr{H}^{1}=: c
$$

where we make use of the generalised Gauss-Green formula (cf. [1] Theorem 3.36). Put $X:=(1 / c) Y$. Then $X \in C_{c}^{\infty}\left(\mathbb{R}^{2}, \mathbb{R}^{2}\right)$ with $\operatorname{supp}[X] \subset B(p, r)$ and $\delta V_{f}(X)=1$ according to Proposition 3.2.

Proposition 3.5 Let $f$ be a positive lower semi-continuous density on $\mathbb{R}^{2}$. Let $U$ be a bounded open set in $\mathbb{R}^{2}$ with Lipschitz boundary. Let $E, F_{1}, F_{2}$ be bounded sets in $\mathbb{R}^{2}$ with finite perimeter. Assume that $E \Delta F_{1} \subset \subset U$ and $E \Delta F_{2} \subset \subset \mathbb{R}^{2} \backslash \bar{U}$. Define

$$
F:=\left[F_{1} \cap U\right] \cup\left[F_{2} \backslash U\right] .
$$


Then $F$ is a set of finite perimeter in $\mathbb{R}^{2}$ and

$$
P_{f}(E)+P_{f}(F)=P_{f}\left(F_{1}\right)+P_{f}\left(F_{2}\right)
$$

Proof The function $\left.\chi_{E}\right|_{U} \in \mathrm{BV}(U)$ and $D\left(\left.\chi_{E}\right|_{U}\right)=\left.\left(D \chi_{E}\right)\right|_{U}$. We write $\chi_{E}^{U}$ for the boundary trace of $\left.\chi_{E}\right|_{U}$ (see [1] Theorem 3.87); then $\chi_{E}^{U} \in L^{1}\left(\partial U, \mathscr{H}^{1}\llcorner\partial U\right.$ ) (cf. [1] Theorem 3.88). We use similar notation elsewhere. By [1] Corollary 3.89,

$$
\begin{aligned}
& D \chi_{E}=D \chi_{E}\left\llcorner U+\left(\chi_{E}^{U}-\chi_{E}^{\mathbb{R}^{2} \backslash \bar{U}}\right) v^{U} \mathscr{H}^{1}\left\llcorner\partial U+D \chi_{E}\left\llcorner\left(\mathbb{R}^{2} \backslash \bar{U}\right)\right.\right.\right. \\
& D \chi_{F}=D \chi_{F_{1}}\left\llcorner U+\left(\chi_{F_{1}}^{U}-\chi_{F_{2}}^{\mathbb{R}^{2} \backslash \bar{U}}\right) v^{U} \mathscr{H}^{1}\left\llcorner\partial U+D \chi_{F_{2}}\left\llcorner\left(\mathbb{R}^{2} \backslash \bar{U}\right)\right.\right.\right. \\
& D \chi_{F_{1}}=D \chi_{F_{1}}\left\llcorner U+\left(\chi_{F_{1}}^{U}-\chi_{E}^{\mathbb{R}^{2} \backslash \bar{U}}\right) v^{U} \mathscr{H}^{1}\left\llcorner\partial U+D \chi_{E}\left\llcorner\left(\mathbb{R}^{2} \backslash \bar{U}\right)\right.\right.\right. \\
& D \chi_{F_{2}}=D \chi_{E}\left\llcorner U+\left(\chi_{E}^{U}-\chi_{F_{2}}^{\mathbb{R}^{2} \backslash \bar{U}}\right) v^{U} \mathscr{H}^{1}\left\llcorner\partial U+D \chi_{F_{2}}\left\llcorner\left(\mathbb{R}^{2} \backslash \bar{U}\right) .\right.\right.\right.
\end{aligned}
$$

From the definition of the total variation measure ([1] Definition 1.4),

$$
\begin{aligned}
& \left|D \chi_{E}\right|=\left|D \chi_{E}\right|\left\llcorner U+\left|\chi_{E}^{U}-\chi_{E}^{\mathbb{R}^{2} \backslash \bar{U}}\right| \mathscr{H}^{1}\left\llcorner\partial U+\left|D \chi_{E}\right|\left\llcorner\left(\mathbb{R}^{2} \backslash \bar{U}\right)\right.\right.\right. \\
& \left|D \chi_{F}\right|=\left|D \chi_{F_{1}}\right|\left\llcorner U+\left|\chi_{E}^{U}-\chi_{E}^{\mathbb{R}^{2} \backslash \bar{U}}\right| \mathscr{H}^{1}\left\llcorner\partial U+\left|D \chi_{F_{2}}\right|\left\llcorner\left(\mathbb{R}^{2} \backslash \bar{U}\right)\right.\right.\right. \\
& \left|D \chi_{F_{1}}\right|=\left|D \chi_{F_{1}}\right|\left\llcorner U+\left|\chi_{E}^{U}-\chi_{E}^{\mathbb{R}^{2} \backslash \bar{U}}\right| \mathscr{H}^{1}\left\llcorner\partial U+\left|D \chi_{E}\right|\left\llcorner\left(\mathbb{R}^{2} \backslash \bar{U}\right) ;\right.\right.\right. \\
& \left|D \chi_{F_{2}}\right|=\left|D \chi_{E}\right|\left\llcorner U+\left|\chi_{E}^{U}-\chi_{E}^{\mathbb{R}^{2} \backslash \bar{U}}\right| \mathscr{H}^{1}\left\llcorner\partial U+\left|D \chi_{F_{2}}\right|\left\llcorner\left(\mathbb{R}^{2} \backslash \bar{U}\right)\right.\right.\right.
\end{aligned}
$$

where we also use the fact that $\chi_{F_{1}}^{U}=\chi_{E}^{U}$ as $E \Delta F_{1} \subset \subset U$ and similarly for $F_{2}$. The result now follows.

Proposition 3.6 Assume that $f$ is a positive locally Lipschitz density on $\mathbb{R}^{2}$. Let $v>0$ and suppose that the set $E$ is a bounded minimiser of (1.2). Let $U$ be a bounded open set in $\mathbb{R}^{2}$. There exist constants $C>0$ and $\delta>0$ with the following property. For any $x \in U$ and $0<r<\delta$,

$$
P_{f}(E)-P_{f}(F) \leq C\left|V_{f}(E)-V_{f}(F)\right|
$$

where $F$ is any set with finite perimeter in $\mathbb{R}^{2}$ such that $E \Delta F \subset \subset B(x, r)$.

Proof The proof follows that of [21] Proposition 3.1. We assume to the contrary that

$$
\begin{gathered}
(\forall C>0)(\forall \delta>0)(\exists x \in U)(\exists r \in(0, \delta))\left(\exists F \subset \mathbb{R}^{2}\right) \\
{\left[F \Delta E \subset \subset B(x, r) \wedge \Delta P_{f}>C\left|\Delta V_{f}\right|\right]}
\end{gathered}
$$

in the language of quantifiers where we have taken some liberties with notation. 
Choose $p_{1}, p_{2} \in \mathscr{F} E$ with $p_{1} \neq p_{2}$. Choose $r_{0}>0$ such that the open balls $B\left(p_{1}, r_{0}\right)$ and $B\left(p_{2}, r_{0}\right)$ are disjoint. Choose vector fields $X_{j} \in C_{c}^{\infty}\left(\mathbb{R}^{2}, \mathbb{R}^{2}\right)$ with $\operatorname{supp}\left[X_{j}\right] \subset B\left(p_{j}, r_{0}\right)$ such that

$$
\delta V_{f}\left(X_{j}\right)=1 \text { and }\left|P_{f}\left(\varphi_{t}^{(j)}(E)\right)-P_{f}(E)\right| \leq a_{j}|t| \text { for }|t|<\delta_{j} \text { and } j=1,2
$$

as in Lemma 3.4 and Proposition 3.3. Put $a:=\max \left\{a_{1}, a_{2}\right\}$. By (3.5),

$$
V_{f}\left(\varphi_{t}^{(j)}(E)\right)-V_{f}(E)=t+o(t) \text { as } t \rightarrow 0 \text { for } j=1,2 \text {. }
$$

So there exist $\varepsilon>0$ and $1>\eta>0$ such that

$$
\begin{aligned}
& t-\eta|t|<V_{f}\left(\varphi_{t}^{(j)}(E)\right)-V_{f}(E)<t+\eta|t| \\
& \left|P_{f}\left(\varphi_{t}^{(j)}(E)\right)-P_{f}(E)\right|<(a+1)|t|
\end{aligned}
$$

for $|t|<\varepsilon$ and $j=1,2$. In particular,

$$
\begin{aligned}
& \left|V_{f}\left(\varphi_{t}^{(j)}(E)\right)-V_{f}(E)\right|>(1-\eta)|t| \\
& \left|P_{f}\left(\varphi_{t}^{(j)}(E)\right)-P_{f}(E)\right|<\frac{1+a}{1-\eta}\left|V_{f}\left(\varphi_{t}^{(j)}(E)\right)-V_{f}(E)\right| \text { for }|t|<\varepsilon
\end{aligned}
$$

for $|t|<\varepsilon$ and $j=1,2$.

In (3.4) choose $C=(1+a) /(1-\eta)$ and $\delta>0$ such that

(a) $0<2 \delta<\operatorname{dist}\left(B\left(p_{1}, r_{0}\right), B\left(p_{2}, r_{0}\right)\right)$,

(b) $\sup \left\{V_{f}(B(x, \delta)): x \in U\right\}<(1-\eta) \varepsilon$.

Choose $x, r$ and $F_{1}$ as in (3.4). In light of (a) we may assume that $B(x, r) \cap B\left(p_{1}, r_{0}\right)=$ $\emptyset$. Вy (b),

$$
\left|V_{f}\left(F_{1}\right)-V_{f}(E)\right| \leq V_{f}(B(x, r)) \leq V_{f}(B(x, \delta))<(1-\eta) \varepsilon .
$$

From (3.6) and (3.8) we can find $t \in(-\varepsilon, \varepsilon)$ such that with $F_{2}:=\varphi_{t}^{(1)}(E)$,

$$
V_{f}\left(F_{2}\right)-V_{f}(E)=-\left\{V_{f}\left(F_{1}\right)-V_{f}(E)\right\}
$$

by the intermediate value theorem. From (3.4),

$$
P_{f}\left(F_{1}\right)<P_{f}(E)-C\left|V_{f}\left(F_{1}\right)-V_{f}(E)\right|
$$

while from (3.7),

$$
P_{f}\left(F_{2}\right)<P_{f}(E)+C\left|V_{f}\left(F_{2}\right)-V_{f}(E)\right| .
$$


Let $F$ be the set

$$
\left.F:=\left[F_{1} \backslash B\left(p_{1}, r_{0}\right)\right)\right] \cup\left[B\left(p_{1}, r_{0}\right) \cap F_{2}\right]
$$

Note that $E \Delta F_{2} \subset \subset B\left(p_{1}, r_{0}\right)$. By Proposition $3.5, F$ is a bounded set of finite perimeter in $\mathbb{R}^{2}$ and

$$
P_{f}(E)+P_{f}(F)=P_{f}\left(F_{1}\right)+P_{f}\left(F_{2}\right)
$$

We then infer from (3.10), (3.11) and (3.9) that

$$
\begin{aligned}
P_{f}(F)= & P_{f}\left(F_{1}\right)+P_{f}\left(F_{2}\right)-P_{f}(E) \\
< & P_{f}(E)-C\left|V_{f}\left(F_{1}\right)-V_{f}(E)\right|+P_{f}(E) \\
& +C\left|V_{f}\left(F_{2}\right)-V_{f}(E)\right|-P_{f}(E)=P_{f}(E) .
\end{aligned}
$$

On the other hand, $V_{f}(F)=V_{f}\left(F_{1}\right)+V_{f}\left(F_{2}\right)-V_{f}(E)=V_{f}(E)$ by (3.9). We therefore obtain a contradiction to the $f$-isoperimetric property of $E$.

Let $E$ be a set of finite perimeter in $\mathbb{R}^{2}$ and $U$ a bounded open set in $\mathbb{R}^{2}$. The minimality excess is the function $\psi$ defined by

$$
\psi(E, U):=P(E, U)-v(E, U)
$$

where

$$
v(E, U):=\inf \{P(F, U): F \text { is a set of finite perimeter with } F \Delta E \subset \subset U\}
$$

as in [27] (1.9). We recall that the boundary of $E$ is said to be almost minimal in $\mathbb{R}^{2}$ if for each bounded open set $U$ in $\mathbb{R}^{2}$ there exists $T>0$ and a positive constant $K$ such that for every $x \in U$ and $r \in(0, T)$,

$$
\psi(E, B(x, r)) \leq K r^{2}
$$

This definition corresponds to [27] Definition 1.5.

Theorem 3.7 Assume that $f$ is a positive locally Lipschitz density on $\mathbb{R}^{2}$. Let $v>0$ and assume that $E$ is a bounded minimiser of (1.2). Then the boundary of $E$ is almost minimal in $\mathbb{R}^{2}$.

Proof Let $U$ be a bounded open set in $\mathbb{R}^{2}$ and $C>0$ and $\delta>0$ as in Proposition 3.6. The open $\delta$-neighbourhood of $U$ is denoted $I_{\delta}(U)$. Let $x \in U$ and $r \in(0, \delta)$. Put $V:=I_{2 \delta}(U)$. For the sake of brevity write $m:=\inf _{B(x, r)} f$ and $M:=\sup _{B(x, r)} f$. Let $F$ be a set of finite perimeter in $\mathbb{R}^{2}$ such that $F \Delta E \subset \subset B(x, r)$. By Proposition 3.6, 


$$
\begin{aligned}
P & (E, B(x, r))-P(F, B(x, r)) \\
& \leq \frac{1}{m} P_{f}(E, B(x, r))-\frac{1}{M} P_{f}(F, B(x, r)) \\
& =\frac{1}{m}\left(P_{f}(E, B(x, r))-P_{f}(F, B(x, r))\right)+\left(\frac{1}{m}-\frac{1}{M}\right) P_{f}(F, B(x, r)) \\
& \leq \frac{1}{m}\left(P_{f}(E, B(x, r))-P_{f}(F, B(x, r))\right)+\frac{M-m}{m^{2}} P_{f}(F, B(x, r)) \\
& \leq \frac{C}{\inf _{V} f}\left|V_{f}(E)-V_{f}(F)\right|+(2 L r) \frac{\sup _{V} f}{\left(\inf _{V} f\right)^{2}} P(F, B(x, r)) \\
& \leq C \pi r^{2} \frac{\sup _{V} f}{\inf _{V} f}+(2 L r) \frac{\sup _{V} f}{\left(\inf _{V} f\right)^{2}} P(F, B(x, r))
\end{aligned}
$$

where $L$ stands for the Lipschitz constant of the restriction of $f$ to $V$. We then derive that

$$
\psi(E, B(x, r)) \leq C \pi r^{2} \frac{\sup _{V} f}{\inf _{V} f}+(2 L r) \frac{\sup _{V} f}{\left(\inf _{V} f\right)^{2}} v(E, B(x, r)) .
$$

By [13] (5.14), $v(E, B(x, r)) \leq \pi r$. The inequality in (3.13) now follows.

Theorem 3.8 Assume that $f$ is a positive locally Lipschitz density on $\mathbb{R}^{2}$. Let $v>0$ and suppose that $E$ is a bounded minimiser of (1.2). Then there exists a set $\widetilde{E} \subset \mathbb{R}^{2}$ such that

(i) $\widetilde{E}$ is a bounded minimiser of (1.2);

(ii) $\underset{\mathbb{E}}{\widetilde{E}}$ is equivalent to $E$;

(iii) $\widetilde{E}$ is open and $\partial \widetilde{E}$ is a $C^{1}$ hypersurface in $\mathbb{R}^{2}$.

Proof By [13] Proposition 3.1 there exists a Borel set $F$ equivalent to $E$ with the property that

$$
\partial F=\left\{x \in \mathbb{R}^{2}: 0<|F \cap B(x, \rho)|<\pi \rho^{2} \text { for each } \rho>0\right\} .
$$

By Theorem 3.7 and [27] Theorem 1.9, $\partial F$ is a $C^{1}$ hypersurface in $\mathbb{R}^{2}$ (taking note of differences in notation). The set

$$
\widetilde{E}:=\left\{x \in \mathbb{R}^{2}:|F \cap B(x, \rho)|=\pi \rho^{2} \text { for some } \rho>0\right\}
$$

satisfies $(i)-(i i i)$.

\section{Weakly bounded curvature and spherical cap symmetry}

Theorem 4.1 Assume that $f$ is a positive locally Lipschitz density on $\mathbb{R}^{2}$. Let $v>0$ and suppose that $E$ is a bounded minimiser of (1.2). Then there exists a set $\widetilde{E} \subset \mathbb{R}^{2}$ such that

(i) $\widetilde{E}$ is a bounded minimiser of (1.2);

(ii) $\widetilde{E}$ is equivalent to $E$; 
(iii) $\widetilde{E}$ is open and $\partial \widetilde{E}$ is a $C^{1,1}$ hypersurface in $\mathbb{R}^{2}$.

Proof We may assume that $E$ has the properties listed in Theorem 3.8. Put $M:=\partial E$. Let $x \in M$ and $U$ a bounded open set containing $x$. Choose $C>0$ and $\delta>0$ as in Proposition 3.6. Let $0<r<\delta$ and $X \in C_{c}^{\infty}\left(\mathbb{R}^{2}, \mathbb{R}^{2}\right)$ with $\operatorname{supp}[X] \subset B(x, r)$. Then

$$
P_{f}(E)-P_{f}\left(\varphi_{t}(E)\right) \leq C\left|V_{f}(E)-V_{f}\left(\varphi_{t}(E)\right)\right|
$$

for each $t \in \mathbb{R}$. From the identity (3.2),

$$
\begin{aligned}
& -\int_{M}\left(f \circ \varphi_{t}\right)\left(J_{1} d^{M}\left(\varphi_{t}\right)_{x}-1\right) d \mathscr{H}^{1} \leq C\left|V_{f}(E)-V_{f}\left(\varphi_{t}(E)\right)\right| \\
& \quad+\int_{M}\left[f \circ \varphi_{t}-f\right] d \mathscr{H}^{1} \\
& \leq C\left|V_{f}(E)-V_{f}\left(\varphi_{t}(E)\right)\right|+\sqrt{2} K\|X\|_{\infty} \mathscr{H}^{1}(M \cap \operatorname{supp}[X]) t
\end{aligned}
$$

where $K$ stands for the Lipschitz constant of $f$ restricted to $U$. On dividing by $t$ and taking the limit $t \rightarrow 0$ we obtain

$$
\begin{aligned}
-\int_{M} f \operatorname{div}^{M} X d \mathscr{H}^{1} \leq & C\left|\int_{M} f\langle n, X\rangle d \mathscr{H}^{1}\right| \\
& +\sqrt{2} K\|X\|_{\infty} \mathscr{H}^{1}(M \cap \operatorname{supp}[X])
\end{aligned}
$$

upon using Lemma 2.2 and Proposition 3.2. Replacing $X$ by $-X$ we derive that

$$
\left|\int_{M} f \operatorname{div}^{M} X d \mathscr{H}^{1}\right| \leq C_{1}\|X\|_{\infty} \mathscr{H}^{1}(M \cap \operatorname{supp}[X])
$$

where $C_{1}=C\|f\|_{L^{\infty}(U)}+\sqrt{2} K$. Let $\gamma_{1}: I \rightarrow M$ be a local $C^{1}$ parametrisation of $M$ near $x$. Suppose that $Y \in C_{c}^{1}\left(I, \mathbb{R}^{2}\right)$ with supp $[Y] \subset I$ and that $\gamma_{1}(I) \subset M \cap B(x, r)$. Note that there exists $X \in C_{c}^{\infty}\left(\mathbb{R}^{2}, \mathbb{R}^{2}\right)$ with $\operatorname{supp}[X] \subset B(x, r)$ such that $X \circ \gamma_{1}=Y$ on $I$. The above estimate entails that

$$
\left|\int_{I}\left(f \circ \gamma_{1}\right)\langle\dot{Y}, t\rangle d s\right| \leq C_{1}|\operatorname{supp}[Y]|\|Y\|_{\infty}
$$

This means that the function $\left(f \circ \gamma_{1}\right) t$ belongs to $\mathrm{BV}(I)$ and this implies in turn that $t \in \mathrm{BV}(I)$. For $s_{1}, s_{2} \in I$ with $s_{1}<s_{2}$,

$$
\begin{aligned}
\left|t\left(s_{2}\right)-t\left(s_{1}\right)\right| & =\left|D t\left(\left(s_{1}, s_{2}\right)\right)\right| \leq|D t|\left(\left(s_{1}, s_{2}\right)\right) \\
& =\sup \left\{\int_{\left(s_{1}, s_{2}\right)}\langle t, \dot{Y}\rangle d s: Y \in C_{c}^{1}\left(\left(s_{1}, s_{2}\right)\right) \text { and }\|Y\|_{\infty} \leq 1\right\} \\
& \leq c \sup \left\{\int_{\left(s_{1}, s_{2}\right)}\left(f \circ \gamma_{1}\right)\langle t, \dot{Y}\rangle d s: Y \in C_{c}^{1}\left(\left(s_{1}, s_{2}\right)\right) \text { and }\|Y\|_{\infty} \leq 1\right\} \\
& \leq c C_{1}\left|s_{2}-s_{1}\right|
\end{aligned}
$$


where $1 / c=\inf _{\bar{U}} f>0$. It follows that $M$ is of class $C^{1,1}$.

We turn to the topic of spherical cap symmetrisation. Denote by $\mathbb{S}_{\tau}^{1}$ the centred circle in $\mathbb{R}^{2}$ with radius $\tau>0$. We sometimes write $\mathbb{S}^{1}$ for $\mathbb{S}_{1}^{1}$. Given $x \in \mathbb{R}^{2}, v \in \mathbb{S}^{1}$ and $\alpha \in(0, \pi]$ the open cone with vertex $x$, axis $v$ and opening angle $2 \alpha$ is the set

$$
C(x, v, \alpha):=\left\{y \in \mathbb{R}^{2}:\langle y-x, v\rangle>|y-x| \cos \alpha\right\} .
$$

Let $E$ be an $\mathscr{L}^{2}$-measurable set in $\mathbb{R}^{2}$ and $\tau>0$. The $\tau$-section $E_{\tau}$ of $E$ is the set $E_{\tau}:=E \cap \mathbb{S}_{\tau}^{1}$. Put

$$
L(\tau)=L_{E}(\tau):=\mathscr{H}^{1}\left(E_{\tau}\right) \text { for } \tau>0
$$

and $p(E):=\{\tau>0: L(\tau)>0\}$. The function $L$ is $\mathscr{L}^{1}$-measurable by [1] Theorem 2.93. Given $\tau>0$ and $0<\alpha \leq \pi$ the spherical cap $C(\tau, \alpha)$ is the set

$$
C(\tau, \alpha):= \begin{cases}\mathbb{S}_{\tau}^{1} \cap C\left(0, e_{1}, \alpha\right) & \text { if } 0<\alpha<\pi \\ \mathbb{S}_{\tau}^{1} & \text { if } \alpha=\pi\end{cases}
$$

and has $\mathscr{H}^{1}$-measure $s(\tau, \alpha):=2 \alpha \tau$. The spherical cap symmetral $E^{s c}$ of the set $E$ is defined by

$$
E^{s c}:=\bigcup_{\tau \in p(E)} C(\tau, \alpha)
$$

where $\alpha \in(0, \pi]$ is determined by $s(\tau, \alpha)=L(\tau)$. Observe that $E^{s c}$ is a $\mathscr{L}^{2}$ measurable set in $\mathbb{R}^{2}$ and $V_{f}\left(E^{s c}\right)=V_{f}(E)$. Note also that if $B$ is a centred open ball then $B^{s c}=B \backslash\{0\}$. We say that $E$ is spherical cap symmetric if $\mathscr{H}^{1}\left(\left(E \Delta E^{s c}\right)_{\tau}\right)=0$ for each $\tau>0$. This definition is broad but suits our purposes.

The result below is stated in [22] Theorem 6.2 and a sketch proof given. A proof along the lines of [2] Theorem 1.1 can be found in [23]. First, let $B$ be a Borel set in $(0,+\infty)$; then the annulus $A(B)$ over $B$ is the set $A(B):=\left\{x \in \mathbb{R}^{2}:|x| \in B\right\}$.

Theorem 4.2 Let $E$ be a set of finite perimeter in $\mathbb{R}^{2}$. Then $E^{\text {sc }}$ is a set of finite perimeter and

$$
P\left(E^{s c}, A(B)\right) \leq P(E, A(B))
$$

for any Borel set $B \subset(0, \infty)$ and the same inequality holds with $E^{s c}$ replaced by any set $F$ that is $\mathscr{L}^{2}$-equivalent to $E^{s c}$.

Corollary 4.3 Let $f$ be a positive lower semi-continuous radial function on $\mathbb{R}^{2}$. Let $E$ be a set of finite perimeter in $\mathbb{R}^{2}$. Then $P_{f}\left(E^{s c}\right) \leq P_{f}(E)$.

Proof Assume that $P_{f}(E)<+\infty$. We remark that $f$ is Borel measurable as $f$ is lower semi-continuous. Let $\left(f_{h}\right)$ be a sequence of simple Borel measurable radial 
functions on $\mathbb{R}^{2}$ such that $0 \leq f_{h} \leq f$ and $f_{h} \uparrow f$ on $\mathbb{R}^{2}$ as $h \rightarrow \infty$. By Theorem 4.2 ,

$$
P_{f_{h}}\left(E^{s c}\right)=\int_{\mathbb{R}^{2}} f_{h} d\left|D \chi_{E^{s c}}\right| \leq \int_{\mathbb{R}^{2}} f_{h} d\left|D \chi_{E}\right|=P_{f_{h}}(E)
$$

for each $h$. Taking the limit $h \rightarrow \infty$ the monotone convergence theorem gives $P_{f}\left(E^{s c}\right) \leq P_{f}(E)$.

Lemma 4.4 Let $E$ be an $\mathscr{L}^{2}$-measurable set in $\mathbb{R}^{2}$ such that $E \backslash\{0\}=E^{s c}$. Then there exists an $\mathscr{L}^{2}$-measurable set $F$ equivalent to $E$ such that

(i) $\partial F=\left\{x \in \mathbb{R}^{2}: 0<|F \cap B(x, \rho)|<|B(x, \rho)|\right.$ for any $\left.\rho>0\right\}$;

(ii) $F$ is spherical cap symmetric.

Proof Put

$$
\begin{aligned}
& E_{1}:=\left\{x \in \mathbb{R}^{2}:|E \cap B(x, \rho)|=|B(x, \rho)| \text { for some } \rho>0\right\} \\
& E_{0}:=\left\{x \in \mathbb{R}^{2}:|E \cap B(x, \rho)|=0 \text { for some } \rho>0\right\} .
\end{aligned}
$$

We claim that $E_{1}$ is spherical cap symmetric. For take $x \in E_{1}$ with $\tau=|x|>0$ and $|\theta(x)| \in(0, \pi]$. Now $|E \cap B(x, \rho)|=|B(x, \rho)|$ for some $\rho>0$. Let $y \in \mathbb{R}^{2}$ with $|y|=\tau$ and $|\theta(y)|<|\theta(x)|$. Choose a rotation $O \in \operatorname{SO}(2)$ such that $O B(x, \rho)=$ $B(y, \rho)$. As $E \backslash\{0\}=E^{s c},|E \cap B(y, \rho)|=|O(E \cap B(x, \rho))|=|E \cap B(x, \rho)|=$ $|B(x, \rho)|=|B(y, \rho)|$. The claim follows. It follows in a similar way that $\mathbb{R}^{2} \backslash E_{0}$ is spherical cap symmetric. It can then be seen that the set $F:=\left(E_{1} \cup E\right) \backslash E_{0}$ inherits this property. As in [13] Proposition 3.1 the set $F$ is equivalent to $E$ and enjoys the property in $(i)$.

Theorem 4.5 Let $f$ be as in (1.3) where $h:[0,+\infty) \rightarrow \mathbb{R}$ is a non-decreasing convex function. Given $v>0$ let $E$ be a bounded minimiser of (1.2). Then there exists an $\mathscr{L}^{2}$-measurable set $\widetilde{E}$ with the properties

(i) $\widetilde{E}$ is a minimiser of (1.2);

(ii) $L_{\widetilde{E}}=$ La.e. on $(0,+\infty)$;

(iii) $\widetilde{E}$ is open, bounded and has $C^{1,1}$ boundary;

(iv) $\widetilde{E} \backslash\{0\}=\widetilde{E}^{s c}$.

Proof Let $E$ be a bounded minimiser for (1.2). Then $E_{1}:=E^{s c}$ is a bounded minimiser of (1.2) by Corollary 4.3 and $L_{E}=L_{E_{1}}$ on $(0,+\infty)$. Now put $E_{2}:=F$ with $F$ as in Lemma 4.4. Then $L_{E_{2}}=L$ a.e. on $(0,+\infty)$ as $E_{2}$ is equivalent to $E_{1}, E_{2}$ is a bounded minimiser of (1.2) and $E_{2}$ is spherical cap symmetric. Moreover, $\partial E_{2}=\left\{x \in \mathbb{R}^{2}\right.$ : $0<\left|E_{2} \cap B(x, \rho)\right|<|B(x, \rho)|$ for any $\left.\rho>0\right\}$. As in the proof of Theorem 3.8, $\partial E_{2}$ is a $C^{1}$ hypersurface in $\mathbb{R}^{2}$. Put

$$
\widetilde{E}:=\left\{x \in \mathbb{R}^{2}:\left|E_{2} \cap B(x, \rho)\right|=|B(x, \rho)| \text { for some } \rho>0\right\} .
$$

Then $\widetilde{E}$ is equivalent to $E_{2}$ so that (ii) holds, and is a bounded minimiser of (1.2); $\widetilde{E}$ is open and $\partial \widetilde{E}=\partial E_{2}$ is $C^{1}$. In fact, $\partial \widetilde{E}$ is of class $C^{1,1}$ by Theorem 4.1. As $E_{2}$ 
is spherical cap symmetric the same is true of $\widetilde{E}$. But $\widetilde{E}$ is open which entails that $\widetilde{E} \backslash\{0\}=\widetilde{E}^{s c}$.

\section{More on spherical cap symmetry}

Let

$$
H:=\left\{x=\left(x_{1}, x_{2}\right) \in \mathbb{R}^{2}: x_{2}>0\right\}
$$

stand for the open upper half-plane in $\mathbb{R}^{2}$ and

$$
S: \mathbb{R}^{2} \rightarrow \mathbb{R}^{2} ; x=\left(x_{1}, x_{2}\right) \mapsto\left(x_{1},-x_{2}\right)
$$

for reflection in the $x_{1}$-axis. Let $O \in \mathrm{SO}(2)$ represent rotation anti-clockwise through $\pi / 2$.

Lemma 5.1 Let $E$ be an open set in $\mathbb{R}^{2}$ with $C^{1}$ boundary $M$ and assume that $E \backslash\{0\}=$ $E^{s c}$. Let $x \in M \backslash\{0\}$. Then

(i) $S x \in M \backslash\{0\}$;

(ii) $n(S x)=S n(x)$;

(iii) $\cos \sigma(S x)=-\cos \sigma(x)$.

Proof (i) The closure $\bar{E}$ of $E$ is spherical cap symmetric. The spherical cap symmetral $\bar{E}$ is invariant under $S$ from the representation (4.2). (ii) is a consequence of this last observation. (iii) Note that $t(S x)=O^{\star} n(S x)=O^{\star} S n(x)$. Then

$$
\begin{aligned}
\cos \sigma(S x) & =\langle S x, t(S x)\rangle=\left\langle S x, O^{\star} S n(x)\right\rangle=\left\langle x, S O^{\star} S n(x)\right\rangle \\
& =\langle x, O n(x)\rangle=-\left\langle x, O^{\star} n(x)\right\rangle=\cos \sigma(x)
\end{aligned}
$$

as $S O^{\star} S=O$ and $O=-O^{\star}$.

We introduce the projection $\pi: \mathbb{R}^{2} \rightarrow[0,+\infty) ; x \mapsto|x|$.

Lemma 5.2 Let $E$ be an open set in $\mathbb{R}^{2}$ with boundary $M$ and assume that $E \backslash\{0\}=$ $E^{s c}$.

(i) Suppose $0 \neq x \in \mathbb{R}^{2} \backslash \bar{E}$ and $\theta(x) \in(0, \pi]$. Then there exists an open interval $I$ in $(0,+\infty)$ containing $\tau$ and $\alpha \in(0, \theta(x))$ such that $A(I) \backslash \bar{S}(\alpha) \subset \mathbb{R}^{2} \backslash \bar{E}$.

(ii) Suppose $0 \neq x \in E$ and $\theta(x) \in[0, \pi)$. Then there exists an open interval $I$ in $(0,+\infty)$ containing $\tau$ and $\alpha \in(\theta(x), \pi)$ such that $A(I) \cap S(\alpha) \subset E$.

(iii) For each $0<\tau \in \pi(M), M_{\tau}$ is the union of two closed spherical arcs in $\mathbb{S}_{\tau}^{1}$ symmetric about the $x_{1}$-axis.

Proof (i) We can find $\alpha \in(0, \theta(x))$ such that $\mathbb{S}_{\tau}^{1} \backslash S(\alpha) \subset \mathbb{R}^{2} \backslash \bar{E}$ as can be seen from definition (4.2). This latter set is compact so $\operatorname{dist}\left(\mathbb{S}_{\tau}^{1} \backslash S(\alpha), \bar{E}\right)>0$. This means that the $\varepsilon$-neighbourhood of $\mathbb{S}_{\tau}^{1} \backslash S(\alpha)$ is contained in $\mathbb{R}^{2} \backslash \bar{E}$ for $\varepsilon>0$ small. The claim 
follows. (ii) Again from (4.2) we can find $\alpha \in(\theta(x), \pi)$ such that $\overline{\mathbb{S}_{\tau}^{1} \cap S(\alpha)} \subset E$ and the assertion follows as before.

(iii) Suppose $x_{1}, x_{2}$ are distinct points in $M_{\tau}$ with $0 \leq \theta\left(x_{1}\right)<\theta\left(x_{2}\right) \leq \pi$. Suppose $y$ lies in the interior of the spherical arc joining $x_{1}$ and $x_{2}$. If $y \in \mathbb{R}^{2} \backslash \bar{E}$ then $x_{2} \in \mathbb{R}^{2} \backslash \bar{E}$ by (i) and hence $x_{2} \notin M$. If $y \in E$ we obtain the contradiction that $x_{1} \in E$ by (ii). Therefore $y \in M$. We infer that the closed spherical arc joining $x_{1}$ and $x_{2}$ lies in $M_{\tau}$. The claim follows noting that $M_{\tau}$ is closed.

Lemma 5.3 Let $E$ be an open set in $\mathbb{R}^{2}$ with $C^{1}$ boundary M. Let $x \in M$. Then

$$
\liminf _{\bar{E} \ni y \rightarrow x}\left\langle\frac{y-x}{|y-x|}, n(x)\right\rangle \geq 0 .
$$

Proof Assume for a contradiction that

$$
\liminf _{\bar{E} \ni y \rightarrow x}\left\langle\frac{y-x}{|y-x|}, n(x)\right\rangle \in[-1,0)
$$

There exists $\eta \in(0,1)$ and a sequence $\left(y_{h}\right)$ in $E$ such that $y_{h} \rightarrow x$ as $h \rightarrow \infty$ and

$$
\left\langle\frac{y_{h}-x}{\left|y_{h}-x\right|}, n(x)\right\rangle<-\eta
$$

for each $h \in \mathbb{N}$. Choose $\alpha \in(0, \pi / 2)$ such that $\cos \alpha=\eta$. As $M$ is $C^{1}$ there exists $r>0$ such that

$$
B(x, r) \cap C(x,-n(x), \alpha) \cap E=\emptyset .
$$

By choosing $h$ sufficiently large we can find $y_{h} \in B(x, r)$ with the additional property that $y_{h} \in C(x,-n(x), \alpha)$ by (5.1). We are thus led to a contradiction.

Lemma 5.4 Let $E$ be an open set in $\mathbb{R}^{2}$ with $C^{1}$ boundary $M$ and assume that $E \backslash\{0\}=$ $E^{s c}$. For each $0<\tau \in \pi(M)$,

(i) $|\cos \sigma|$ is constant on $M_{\tau}$;

(ii) $\cos \sigma=0$ on $M_{\tau} \cap\left\{x_{2}=0\right\}$;

(iii) $\langle O x, n(x)\rangle \leq 0$ for $x \in M_{\tau} \cap H$

(iv) $\cos \sigma \leq 0$ on $M_{\tau} \cap H$;

and if $\cos \sigma \not \equiv 0$ on $M_{\tau}$ then

(v) $\tau \in p(E)$;

(vi) $M_{\tau}$ consists of two disjoint singletons in $\mathbb{S}_{\tau}^{1}$ symmetric about the $x_{1}$-axis;

(vii) $L(\tau) \in(0,2 \pi \tau)$;

(viii) $M_{\tau}=\{(\tau \cos (L(\tau) / 2 \tau), \pm \tau \sin (L(\tau) / 2 \tau)\}$.

Proof (i) By Lemma 5.2, $M_{\tau}$ is the union of two closed spherical arcs in $\mathbb{S}_{\tau}^{1}$ symmetric about the $x_{1}$-axis. In case $M_{\tau} \cap \bar{H}$ consists of a singleton the assertion follows from Lemma 5.1. Now suppose that $M_{\tau} \cap \bar{H}$ consists of a spherical arc in $\mathbb{S}_{\tau}^{1}$ with non-empty 
interior. It can be seen that $\cos \sigma$ vanishes on the interior of this arc as $0=r_{1}^{\prime}=\cos \sigma_{1}$ in a local parametrisation by (2.9). By continuity $\cos \sigma=0$ on $M_{\tau}$. (ii) follows from Lemma 5.1. (iii) Let $x \in M_{\tau} \cap H$ so $\theta(x) \in(0, \pi)$. Then $S(\theta(x)) \cap \mathbb{S}_{\tau}^{1} \subset \bar{E}$ as $\bar{E}$ is spherical cap symmetric. Then

$$
0 \leq \lim _{S(\theta(x)) \cap \mathbb{S}_{\tau}^{1} \ni y \rightarrow x}\left\langle\frac{y-x}{|y-x|}, n(x)\right\rangle=-\langle O x, n(x)\rangle
$$

by Lemma 5.3. (iv) The adjoint transformation $O^{\star}$ represents rotation clockwise through $\pi / 2$. Let $x \in M_{\tau} \cap H$. By (iii),

$$
0 \geq\langle O x, n(x)\rangle=\left\langle x, O^{\star} n(x)\right\rangle=\langle x, t(x)\rangle=\tau \cos \sigma(x)
$$

and this leads to the result. (v) As $\cos \sigma \not \equiv 0$ on $M_{\tau}$ we can find $x \in M_{\tau} \cap H$. We claim that $\mathbb{S}_{\tau}^{1} \cap S(\theta(x)) \subset E$. For suppose that $y \in \mathbb{S}_{\tau}^{1} \cap S(\theta(x))$ but $y \notin E$. We may suppose that $0 \leq \theta(y)<\theta(x)<\pi$. If $y \in \mathbb{R}^{2} \backslash \bar{E}$ then $x \in \mathbb{R}^{2} \backslash \bar{E}$ by Lemma 5.2. On the other hand, if $y \in M$ then the spherical arc in $H$ joining $y$ to $x$ is contained in $M$ again by Lemma 5.2. This arc also has non-empty interior in $\mathbb{S}_{\tau}^{1}$. Now $\cos \sigma=0$ on its interior so $\cos (\sigma(x))=0$ by $(i)$ contradicting the hypothesis. A similar argument deals with (vi) and this together with (v) in turn entails (vii) and (viii).

Lemma 5.5 Let $E$ be an open set in $\mathbb{R}^{2}$ with $C^{1}$ boundary $M$ and assume that $E \backslash\{0\}=$ $E^{s c}$. Suppose that $0 \in M$. Then

(i) $(\sin \sigma)(0+)=0$;

(ii) $(\cos \sigma)(0+)=-1$.

Proof (i) Let $\gamma_{1}$ be a $C^{1}$ parametrisation of $M$ in a neighbourhood of 0 with $\gamma_{1}(0)=0$ as above. Then $n(0)=n_{1}(0)=e_{1}$ and hence $t(0)=t_{1}(0)=-e_{2}$. By Taylor's Theorem $\gamma_{1}(s)=\gamma_{1}(0)+t_{1}(0) s+o(s)=-e_{2} s+o(s)$ for $s \in I$. This means that $r_{1}(s)=\left|\gamma_{1}(s)\right|=s+o(s)$ and

$$
\cos \theta_{1}=\frac{\left\langle e_{1}, \gamma_{1}\right\rangle}{r_{1}}=\frac{\left\langle e_{1}, \gamma_{1}\right\rangle}{s} \frac{s}{r_{1}} \rightarrow 0
$$

as $s \rightarrow 0$ which entails that $\left(\cos \theta_{1}\right)(0-)=0$. Now $t_{1}$ is continuous on $I$ so $t_{1}=$ $-e_{2}+o(1)$ and $\cos \alpha_{1}=\left\langle e_{1}, t_{1}\right\rangle=o(1)$. We infer that $\left(\cos \alpha_{1}\right)(0-)=0$. By (2.11), $\cos \alpha_{1}=\cos \sigma_{1} \cos \theta_{1}-\sin \sigma_{1} \sin \theta_{1}$ on $I$ and hence $\left(\sin \sigma_{1}\right)(0-)=0$. We deduce that $(\sin \sigma)(0+)=0$. Item (ii) follows from $(i)$ and Lemma 5.4.

The set

$$
\Omega:=\pi[(M \backslash\{0\}) \cap\{\cos \sigma \neq 0\}]
$$

plays an important rôle in the proof of Theorem 1.1.

Lemma 5.6 Let $E$ be an open set in $\mathbb{R}^{2}$ with $C^{1}$ boundary $M$ and assume that $E \backslash\{0\}=$ $E^{s c}$. Then $\Omega$ is an open set in $(0,+\infty)$. 
Proof Suppose $0<\tau \in \Omega$. Choose $x \in M_{\tau} \cap\{\cos \sigma \neq 0\}$. Let $\gamma_{1}: I \rightarrow M$ be a local $C^{1}$ parametrisation of $M$ in a neighbourhood of $x$ such that $\gamma_{1}(0)=x$ as before. By shrinking $I$ if necessary we may assume that $r_{1} \neq 0$ and $\cos \sigma_{1} \neq 0$ on $I$. Then the set $\left\{r_{1}(s): s \in I\right\} \subset \Omega$ is connected and so an interval in $\mathbb{R}$ (see for example [25] Theorems 6.A and 6.B). By (2.9), $r_{1}^{\prime}(0)=\cos \sigma_{1}(0)=\cos \sigma(p) \neq 0$. This means that the set $\left\{r_{1}(s): s \in I\right\}$ contains an open interval about $\tau$.

\section{Generalised (mean) curvature}

Given a set $E$ of finite perimeter in $\mathbb{R}^{2}$ the first variation $\delta V_{f}(Z)$ resp. $\delta P_{f}^{+}(Z)$ of weighted volume and perimeter along a time-dependent vector field $Z$ are defined as in (2.13) and (2.14).

Proposition 6.1 Let $f$ be as in (1.3) where $h:[0,+\infty) \rightarrow \mathbb{R}$ is a non-decreasing convex function. Let $E$ be a bounded open set in $\mathbb{R}^{2}$ with $C^{1}$ boundary M. Let $Z$ be a time-dependent vector field. Then

$$
\delta P_{f}^{+}(Z)=\int_{M} f_{+}^{\prime}\left(\cdot, Z_{0}\right)+f \operatorname{div}^{M} Z_{0} d \mathscr{H}^{1}
$$

where $Z_{0}:=Z(0, \cdot) \in C_{c}^{1}\left(\mathbb{R}^{2}, \mathbb{R}^{2}\right)$.

Proof The identity (3.2) holds for each $t \in I$ with $M$ in place of $\mathscr{F} E$. The assertion follows on appealing to Lemma 2.3 and Lemma 2.4 with the help of the dominated convergence theorem.

Given $X, Y \in C_{c}^{\infty}\left(\mathbb{R}^{2}, \mathbb{R}^{2}\right)$ let $\psi$ resp. $\chi$ stand for the 1-parameter group of $C^{\infty}$ diffeomorphisms of $\mathbb{R}^{2}$ associated to the vector fields $X$ resp. $Y$ as in (2.12). Let $I$ be an open interval in $\mathbb{R}$ containing the point 0 . Suppose that the function $\sigma: I \rightarrow \mathbb{R}$ is $C^{1}$. Define a flow via

$$
\varphi: I \times \mathbb{R}^{2} \rightarrow \mathbb{R}^{2} ;(t, x) \mapsto \chi(\sigma(t), \psi(t, x))
$$

Lemma 6.2 The time-dependent vector field $Z$ associated with the flow $\varphi$ is given by

$$
Z(t, x)=\sigma^{\prime}(t) Y(\chi(\sigma(t), \psi(t, x)))+d \chi(\sigma(t), \psi(t, x)) X(\psi(t, x))
$$

for $(t, x) \in I \times \mathbb{R}^{2}$ and satisfies (Z.1) and (Z.2).

Proof For $t \in I$ and $x \in \mathbb{R}^{2}$ we compute using (2.12),

$$
\partial_{t} \varphi(t, x)=\left(\partial_{t} \chi\right)(\sigma(t), \psi(t, x)) \sigma^{\prime}(t)+d \chi(\sigma(t), \psi(t, x)) \partial_{t} \psi(t, x)
$$

and this gives (6.1). Put $K_{1}:=\operatorname{supp}[X], K_{2}:=\operatorname{supp}[Y]$ and $K:=K_{1} \cup K_{2}$. Then (Z.2) holds with this choice of $K$. 
Let $E$ be a bounded open set in $\mathbb{R}^{2}$ with $C^{1}$ boundary $M$. Define $\Lambda:=(M \backslash\{0\}) \cap$ $\{\cos \sigma=0\}$ and

$$
\Lambda_{1}:=\left\{x \in M: \mathscr{H}^{1}(\Lambda \cap B(x, \rho))=\mathscr{H}^{1}(M \cap B(x, \rho)) \text { for some } \rho>0\right\} .
$$

For future reference put $\Lambda_{1}^{ \pm}:=\Lambda_{1} \cap\{x \in M: \pm\langle x, n\rangle>0\}$.

Lemma 6.3 Let $f$ be as in (1.3) where $h:[0,+\infty) \rightarrow \mathbb{R}$ is a non-decreasing convex function. Let $E$ be a bounded open set in $\mathbb{R}^{2}$ with $C^{1,1}$ boundary $M$ and suppose that $E \backslash\{0\}=E^{s c}$. Then

(i) $\Lambda_{1}$ is a countable disjoint union of well-separated open circular arcs centred at 0 ;

(ii) $\mathscr{H}^{1}\left(\overline{\Lambda_{1}} \backslash \Lambda_{1}\right)=0$;

(iii) $f$ is differentiable $\mathscr{H}^{1}$-a.e. on $M \backslash \overline{\Lambda_{1}}$.

The term well-separated in (i) means the following: if $\Gamma$ is an open circular arc in $\Lambda_{1}$ with $\Gamma \cap\left(\Lambda_{1} \backslash \Gamma\right)=\emptyset$ then $d\left(\Gamma, \Lambda_{1} \backslash \Gamma\right)>0$.

Proof (i) Let $x \in \Lambda_{1}$ and $\gamma_{1}: I \rightarrow M$ a $C^{1,1}$ parametrisation of $M$ near $x$. By shrinking $I$ if necessary we may assume that $\gamma_{1}(I) \subset M \cap B(x, \rho)$ with $\rho$ as in (6.2). So $\cos \sigma=0 \mathscr{H}^{1}$-a.e. on $\gamma_{1}(I)$ and hence $\cos \sigma_{1}=0$ a.e. on $I$. This means that $\cos \sigma_{1}=0$ on $I$ as $\sigma_{1} \in C^{0,1}(I)$ and that $r_{1}$ is constant on $I$ by (2.9). Using (2.10) it can be seen that $\gamma_{1}(I)$ is an open circular arc centred at 0 . By compactness of $M$ it follows that $\Lambda_{1}$ is a countable disjoint union of open circular arcs centred on 0 . The wellseparated property flows from the fact that $M$ is $C^{1}$. (ii) follows as a consequence of this property. (iii) Let $x \in M \backslash \overline{\Lambda_{1}}$ and $\gamma_{1}: I \rightarrow M$ a $C^{1,1}$ parametrisation of $M$ near $x$ with properties as before. We assume that $x$ lies in the upper half-plane $H$. By shrinking $I$ if necessary we may assume that $\gamma_{1}(I) \subset\left(M \backslash \overline{\Lambda_{1}}\right) \cap H$. Let $s_{1}, s_{2}, s_{3} \in I$ with $s_{1}<s_{2}<s_{3}$. Then $y:=\gamma_{1}\left(s_{2}\right) \in M \backslash \overline{\Lambda_{1}}$. So $\mathscr{H}^{1}(M \cap\{\cos \sigma \neq 0\} \cap B(y, \rho))>0$ for each $\rho>0$. This means that for small $\eta>0$ the set $\gamma_{1}\left(\left(s_{2}-\eta, s_{2}+\eta\right)\right) \cap\{\cos \sigma \neq 0\}$ has positive $\mathscr{H}^{1}$-measure. Consequently, $r_{1}\left(s_{3}\right)-r_{1}\left(s_{1}\right)=\int_{s_{1}}^{s_{3}} \cos \sigma_{1} d s<0$ bearing in mind Lemma 5.4. This shows that $r_{1}$ is strictly decreasing on $I$. So $h$ is differentiable a.e. on $r_{1}(I) \subset(0,+\infty)$ in virtue of the fact that $h$ is convex and hence locally Lipschitz. This entails (iii).

Proposition 6.4 Let $f$ be as in (1.3) where $h:[0,+\infty) \rightarrow \mathbb{R}$ is a non-decreasing convex function. Given $v>0$ let $E$ be a minimiser of (1.2). Assume that $E$ is a bounded open set in $\mathbb{R}^{2}$ with $C^{1}$ boundary $M$ and suppose that $E \backslash\{0\}=E^{s c}$. Suppose that $M \backslash \overline{\Lambda_{1}} \neq \emptyset$. Then there exists $\lambda \in \mathbb{R}$ such that for any $X \in C_{c}^{1}\left(\mathbb{R}^{2}, \mathbb{R}^{2}\right)$,

$$
0 \leq \int_{M}\left\{f_{+}^{\prime}(\cdot, X)+f \operatorname{div}^{M} X-\lambda f\langle n, X\rangle\right\} d \mathscr{H}^{1}
$$

Proof Let $X \in C_{c}^{\infty}\left(\mathbb{R}^{2}, \mathbb{R}^{2}\right)$. Let $x \in M$ and $r>0$ such that $M \cap B(x, r) \subset M \backslash \overline{\Lambda_{1}}$. Choose $Y \in C_{c}^{\infty}\left(\mathbb{R}^{2}, \mathbb{R}^{2}\right)$ with $\operatorname{supp}[Y] \subset B(x, r)$ as in Lemma 3.4. Let $\psi$ resp. $\chi$ stand for the 1-parameter group of $C^{\infty}$ diffeomorphisms of $\mathbb{R}^{2}$ associated to the vector 
fields $X$ resp. $Y$ as in (2.12). For each $(s, t) \in \mathbb{R}^{2}$ the set $\chi_{s}\left(\psi_{t}(E)\right)$ is an open set in $\mathbb{R}^{2}$ with $C^{1}$ boundary and $\partial\left(\chi_{s} \circ \psi_{t}\right)(E)=\left(\chi_{s} \circ \psi_{t}\right)(M)$ by Lemma 2.1 . Define

$$
\begin{aligned}
& V(s, t):=V_{f}\left(\chi_{t}\left(\psi_{s}(E)\right)\right)-V_{f}(E), \\
& P(s, t):=P_{f}\left(\chi_{t}\left(\psi_{s}(E)\right)\right),
\end{aligned}
$$

for $(s, t) \in \mathbb{R}^{2}$. We write $F=\left(\chi_{t} \circ \psi_{s}\right)(E)$. Arguing as in Proposition 3.2,

$$
\begin{aligned}
\partial_{t} V(s, t) & =\lim _{h \rightarrow 0}(1 / h)\left\{V_{f}\left(\chi_{h}(F)\right)-V_{f}(F)\right\}=\int_{F} \operatorname{div}(f Y) d x \\
& =\int_{E}\left(\operatorname{div}(f Y) \circ \chi_{t} \circ \psi_{s}\right) J_{2} d\left(\chi_{t} \circ \psi_{s}\right)_{x} d x
\end{aligned}
$$

with an application of the area formula (cf. [1] Theorem 2.71). This last varies continuously in $(s, t)$. The same holds for partial differentiation with respect to $s$. Indeed, put $\eta:=\chi_{t} \circ \psi_{s}$. Then noting that $J_{2} d\left(\eta \circ \psi_{h}\right)=\left(J_{2} d \eta\right) \circ \psi_{h} J_{2} d \psi_{h}$ and using the dominated convergence theorem,

$$
\begin{aligned}
\partial_{s} V(s, t)= & \lim _{h \rightarrow 0}(1 / h)\left\{V_{f}\left(\eta\left(\psi_{h}(E)\right)\right)-V_{f}(\eta(E))\right\} \\
= & \lim _{h \rightarrow 0}(1 / h)\left\{\int_{E}\left(f \circ \eta \circ \psi_{h}\right) J_{2} d\left(\eta \circ \psi_{h}\right)_{x} d x-\int_{E}(f \circ \eta) J_{2} d \eta_{x} d x\right\} \\
= & \lim _{h \rightarrow 0}(1 / h)\left\{\int_{E}\left[\left(f \circ \eta \circ \psi_{h}\right)-(f \circ \eta)\right] J_{2} d\left(\eta \circ \psi_{h}\right)_{x} d x\right. \\
& +\int_{E}(f \circ \eta)\left[\left(J_{2} d \eta \circ \psi_{h}-J_{2} d \eta\right] J_{2} d \psi_{h} d x\right. \\
& \left.+\int_{E}(f \circ \eta) J_{2} d \eta\left[J_{2} d \psi_{h}-1\right] d x\right\} \\
= & \int_{E}\langle\nabla(f \circ \eta), X\rangle J_{2} d \eta_{x} d x+\int_{E}(f \circ \eta)\left\langle\nabla J_{2} d \eta, X\right\rangle d x \\
& +\int_{E}(f \circ \eta) J_{2} d \eta \operatorname{div} X d x
\end{aligned}
$$

where the explanation for the last term can be found in the proof of Proposition 3.2. In this regard we note that $d\left(d \chi_{t}\right)$ (for example) is continuous on $I \times \mathbb{R}^{2}$ (cf. [1] Theorem 3.3 and Exercise 3.2) and in particular $\nabla J_{2} d \chi_{t}$ is continuous on $I \times \mathbb{R}^{2}$. The expression above also varies continuously in $(s, t)$ as can be seen with the help of the dominated convergence theorem. This means that $V(\cdot, \cdot)$ is continuously differentiable on $\mathbb{R}^{2}$. Note that

$$
\partial_{t} V(0,0)=\int_{E} \operatorname{div}(f Y) d x=1
$$


by choice of $Y$. By the implicit function theorem there exists $\eta>0$ and a $C^{1}$ function $\sigma:(-\eta, \eta) \rightarrow \mathbb{R}$ such that $\sigma(0)=0$ and $V(s, \sigma(s))=0$ for $s \in(-\eta, \eta)$; moreover,

$$
\begin{aligned}
\sigma^{\prime}(0) & =-\partial_{S} V(0,0)=-\int_{E}\{\langle\nabla f, X\rangle+f \operatorname{div} X\} d x \\
& =-\int_{E} \operatorname{div}(f X) d x=\int_{M} f\langle n, X\rangle d \mathscr{H}^{1}
\end{aligned}
$$

by the Gauss-Green formula (cf. [1] Theorem 3.36).

The mapping

$$
\varphi:(-\eta, \eta) \times \mathbb{R}^{2} \rightarrow \mathbb{R}^{2} ; t \mapsto \chi(\sigma(t), \psi(t, x))
$$

satisfies conditions (F.1)-(F.4) above with $I=(-\eta, \eta)$ where the associated timedependent vector field $Z$ is given as in (6.1) and satisfies (Z.1) and (Z.2); moreover, $Z_{0}=Z(0, \cdot)=\sigma^{\prime}(0) Y+X$. Note that $Z_{0}=X$ on $M \backslash B(x, r)$.

The mapping $I \rightarrow \mathbb{R} ; t \mapsto P_{f}\left(\varphi_{t}(E)\right)$ is right-differentiable at $t=0$ as can be seen from Proposition 6.1 and has non-negative right-derivative there. By Proposition 6.1 and Lemma 6.3,

$$
\begin{aligned}
0 \leq \delta P_{f}^{+}(Z)= & \int_{M} f_{+}^{\prime}\left(\cdot, Z_{0}\right)+f \operatorname{div}^{M} Z_{0} d \mathscr{H}^{1} \\
= & \int_{M \backslash \overline{\Lambda_{1}}} f_{+}^{\prime}\left(\cdot, Z_{0}\right)+f \operatorname{div}^{M} Z_{0} d \mathscr{H}^{1} \\
& +\int_{\overline{\Lambda_{1}}} f_{+}^{\prime}(\cdot, X)+f \operatorname{div}^{M} X d \mathscr{H}^{1} \\
= & \int_{M \backslash \overline{\Lambda_{1}}} \sigma^{\prime}(0)\langle\nabla f, Y\rangle+\langle\nabla f, X\rangle \\
& +\sigma^{\prime}(0) f \operatorname{div}^{M} Y+f \operatorname{div}^{M} X d \mathscr{H}^{1} \\
& +\int_{\overline{\Lambda_{1}}} f_{+}^{\prime}(\cdot, X)+f \operatorname{div}^{M} X d \mathscr{H}^{1} \\
= & \int_{M} f_{+}^{\prime}(\cdot, X)+f \operatorname{div}^{M} X d \mathscr{H}^{1} \\
& +\sigma^{\prime}(0) \int_{M} f_{+}^{\prime}(\cdot, Y)+f \operatorname{div}^{M} Y d \mathscr{H}^{1} .
\end{aligned}
$$

The identity then follows upon inserting the expression for $\sigma^{\prime}(0)$ above with $\lambda=$ $-\int_{M} f_{+}^{\prime}(\cdot, Y)+f \operatorname{div}^{M} Y d \mathscr{H}^{1}$. The claim follows for $X \in C_{c}^{1}\left(\mathbb{R}^{2}, \mathbb{R}^{2}\right)$ by a density argument.

Theorem 6.5 Let $f$ be as in (1.3) where $h:[0,+\infty) \rightarrow \mathbb{R}$ is a non-decreasing convex function. Given $v>0$ let $E$ be a minimiser of (1.2). Assume that $E$ is a bounded open set in $\mathbb{R}^{2}$ with $C^{1,1}$ boundary $M$ and suppose that $E \backslash\{0\}=E^{s c}$. Suppose that $M \backslash \overline{\Lambda_{1}} \neq \varnothing$. Then there exists $\lambda \in \mathbb{R}$ such that 
(i) $k+\rho \sin \sigma+\lambda=0 \mathscr{H}^{1}$-a.e. on $M \backslash \overline{\Lambda_{1}}$;

(ii) $\rho_{-}-\lambda \leq k \leq \rho_{+}-\lambda$ on $\Lambda_{1}^{+}$;

(iii) $-\rho_{+}-\lambda \leq k \leq-\rho_{-}-\lambda$ on $\Lambda_{1}^{-}$.

The expression $k+\rho \sin \sigma$ is called the generalised (mean) curvature of $M$.

Proof (i) Let $x \in M$ and $r>0$ such that $M \cap B(x, r) \subset M \backslash \overline{\Lambda_{1}}$. Choose $X \in C_{c}^{1}\left(\mathbb{R}^{2}, \mathbb{R}^{2}\right)$ with supp $[X] \subset B(x, r)$. We know from Lemma 6.3 that $f$ is differentiable $\mathscr{H}^{1}$-a.e. on $\operatorname{supp}[X]$. Let $\lambda$ be as in Proposition 6.4. Replacing $X$ by $-X$ we deduce from Proposition 6.4 that

$$
0=\int_{M}\left\{\langle\nabla f, X\rangle+f \operatorname{div}^{M} X-\lambda f\langle n, X\rangle\right\} d \mathscr{H}^{1} .
$$

The divergence theorem on manifolds (cf. [1] Theorem 7.34) holds also for $C^{1,1}$ manifolds. So

$$
\begin{aligned}
\int_{M}\langle\nabla f, X\rangle+f \operatorname{div}^{M} X d \mathscr{H}^{1} & =\int_{M} \partial_{n} f\langle n, X\rangle+\left\langle\nabla^{M} f, X\right\rangle+f \operatorname{div}^{M} X d \mathscr{H}^{1} \\
& =\int_{M} \partial_{n} f\langle n, X\rangle+\operatorname{div}^{M}(f X) d \mathscr{H}^{1} \\
& =\int_{M} \partial_{n} f\langle n, X\rangle-H f\langle n, X\rangle d \mathscr{H}^{1} \\
& =\int_{M} f u\left\{\partial_{n} \log f-H\right\} d \mathscr{H}^{1}
\end{aligned}
$$

where $u=\langle n, X\rangle$. Combining this with the equality above we see that

$$
\int_{M} u f\left\{\partial_{n} \log f-H-\lambda\right\} d \mathscr{H}^{1}=0
$$

for all $X \in C_{c}^{1}\left(\mathbb{R}^{2}, \mathbb{R}^{2}\right)$. This leads to the result.

(ii) Let $x \in M$ and $r>0$ such that $M \cap B(x, r) \subset \Lambda_{1}^{+}$. Let $\phi \in C^{1}\left(\mathbb{S}_{r}^{1}\right)$ with support in $\mathbb{S}_{r}^{1} \cap B(x, r)$. We can construct $X \in C_{c}^{1}\left(\mathbb{R}^{2}, \mathbb{R}^{2}\right)$ with the property that $X=\phi n$ on $M \cap B(x, r)$. By Lemma 2.4,

$$
f_{+}^{\prime}(\cdot, X)=f h_{+}^{\prime}(|x|, \operatorname{sgn}\langle x, X\rangle)|\langle n, X\rangle|=f h_{+}^{\prime}(|x|, \operatorname{sgn} \phi\langle x, n\rangle)|\phi|
$$

on $\Lambda_{1}$. Let us assume that $\phi \geq 0$. As $\langle\cdot, n\rangle>0$ on $\Lambda_{1}^{+}$we have that $f_{+}^{\prime}(\cdot, X)=$ $f \phi h_{+}^{\prime}(|x|,+1)=f \phi \rho_{+}$so by Proposition 6.4,

$$
\begin{aligned}
0 & \leq \int_{M}\left\{f_{+}^{\prime}(\cdot, X)+f \operatorname{div}^{M} X-\lambda f\langle n, X\rangle\right\} d \mathscr{H}^{1} \\
& =\int_{M} f \phi\left\{\rho_{+}-k-\lambda\right\} d \mathscr{H}^{1} .
\end{aligned}
$$


We conclude that $\rho_{+}-k-\lambda \geq 0$ on $M \cap B(x, r)$. Now assume that $\phi \leq 0$. Then $f_{+}^{\prime}(\cdot, X)=-f \phi h_{+}^{\prime}(|x|,-1)=f \phi \rho_{-}$so

$$
0 \leq \int_{M} f \phi\left\{\rho_{-}-k-\lambda\right\} d \mathscr{H}^{1}
$$

and hence $\rho_{-}-k-\lambda \leq 0$ on $M \cap B(x, r)$. This shows (ii).

(iii) The argument is similar. Assume in the first instance that $\phi \geq 0$. Then $f_{+}^{\prime}(\cdot, X)=$ $f \phi h_{+}^{\prime}(|x|,-1)=-f \phi \rho_{-}$so

$$
0 \leq \int_{M} f \phi\left\{-\rho_{-}-k-\lambda\right\} d \mathscr{H}^{1} .
$$

We conclude that $-\rho_{-}-k-\lambda \geq 0$ on $M \cap B(x, r)$. Next suppose that $\phi \leq 0$. Then $f_{+}^{\prime}(\cdot, X)=-f \phi h_{+}^{\prime}(|x|,+1)=-f \phi \rho_{+}$so

$$
0 \leq \int_{M} f \phi\left\{-\rho_{+}-k-\lambda\right\} d \mathscr{H}^{1}
$$

and $-\rho_{+}-k-\lambda \leq 0$ on $M \cap B(x, r)$.

Let $E$ be an open set in $\mathbb{R}^{2}$ with $C^{1}$ boundary $M$ and assume that $E \backslash\{0\}=E^{s c}$ and that $\Omega$ is as in (5.2). Bearing in mind Lemma 5.4 we may define

$$
\begin{aligned}
& \theta_{2}: \Omega \rightarrow(0, \pi) ; \tau \mapsto L(\tau) / 2 \tau ; \\
& \gamma: \Omega \rightarrow M ; \tau \mapsto\left(\tau \cos \theta_{2}(\tau), \tau \sin \theta_{2}(\tau)\right) .
\end{aligned}
$$

The function

$$
u: \Omega \rightarrow[-1,1] ; \tau \mapsto \sin (\sigma(\gamma(\tau)))
$$

plays a key role.

Theorem 6.6 Let $f$ be as in (1.3) where $h:[0,+\infty) \rightarrow \mathbb{R}$ is a non-decreasing convex function. Given $v>0$ let $E$ be a bounded minimiser of (1.2). Assume that $E$ is open with $C^{1,1}$ boundary $M$ and that $E \backslash\{0\}=E^{s c}$. Suppose that $M \backslash \overline{\Lambda_{1}} \neq \varnothing$ and let $\lambda$ be as in Theorem 6.5. Then $u \in C^{0,1}(\Omega)$ and

$$
u^{\prime}+(1 / \tau+\rho) u+\lambda=0
$$

a.e. on $\Omega$.

Proof Let $\tau \in \Omega$ and $x$ a point in the open upper half-plane such that $x \in M_{\tau}$. There exists a $C^{1,1}$ parametrisation $\gamma_{1}: I \rightarrow M$ of $M$ in a neighbourhood of $x$ with $\gamma_{1}(0)=x$ as above. Put $u_{1}:=\sin \sigma_{1}$ on $I$. By shrinking the open interval $I$ if necessary we may assume that $r_{1}: I \rightarrow r_{1}(I)$ is a diffeomorphism and that $r_{1}(I) \subset \subset \Omega$. Note that $\gamma=\gamma_{1} \circ r_{1}^{-1}$ and $u=u_{1} \circ r_{1}^{-1}$ on $r_{1}(I)$. It follows that $u \in C^{0,1}(\Omega)$. By (2.9), 


$$
u^{\prime}=\frac{\dot{u}_{1}}{\dot{r}_{1}} \circ r_{1}^{-1}=\dot{\sigma}_{1} \circ r_{1}^{-1}
$$

a.e. on $r_{1}(I)$. As $\dot{\alpha}_{1}=k_{1}$ a.e. on $I$ and using the identity (2.10) we see that $\dot{\sigma}_{1}=$ $\dot{\alpha}_{1}-\dot{\theta}_{1}=k_{1}-\left(1 / r_{1}\right) \sin \sigma_{1}$ a.e on $I$. Thus,

$$
u^{\prime}=k-(1 / \tau) \sin (\sigma \circ \gamma)=k-(1 / \tau) u
$$

a.e. on $r_{1}(I)$. By Theorem 6.5 there exists $\lambda \in \mathbb{R}$ such that $k+\rho \sin \sigma+\lambda=0$ $\mathscr{H}^{1}$-a.e. on $M$. So

$$
u^{\prime}=-\rho(\tau) u-\lambda-(1 / \tau) u=-(1 / \tau+\rho(\tau)) u-\lambda
$$

a.e. on $r_{1}(I)$. The result follows.

Lemma 6.7 Suppose that $E$ is a bounded open set in $\mathbb{R}^{2}$ with $C^{1}$ boundary $M$ and that $E \backslash\{0\}=E^{s c}$. Then

(i) $\theta_{2} \in C^{1}(\Omega)$;

(ii) $\theta_{2}^{\prime}=-\frac{1}{\tau} \frac{u}{\sqrt{1-u^{2}}}$ on $\Omega$.

Proof Let $\tau \in \Omega$ and $x$ a point in the open upper half-plane such that $x \in M_{\tau}$. There exists a $C^{1}$ parametrisation $\gamma_{1}: I \rightarrow M$ of $M$ in a neighbourhood of $x$ with $\gamma_{1}(0)=x$ as above. By shrinking the open interval $I$ if necessary we may assume that $r_{1}: I \rightarrow r_{1}(I)$ is a diffeomorphism and that $r_{1}(I) \subset \subset \Omega$. It then holds that

$$
\theta_{2}=\theta_{1} \circ r_{1}^{-1} \text { and } \sigma \circ \gamma=\sigma_{1} \circ r_{1}^{-1}
$$

on $r_{1}(I)$ by choosing an appropriate branch of $\theta_{1}$. It follows that $\theta_{2} \in C^{1}(\Omega)$. By the chain-rule, (2.10) and (2.9),

$$
\begin{aligned}
\theta_{2}^{\prime} & =\frac{\dot{\theta}_{1}}{\dot{r}_{1}} \circ r_{1}^{-1}=\left(\frac{1}{r_{1}} \tan \sigma_{1}\right) \circ r_{1}^{-1} \\
& =(1 / \tau) \tan (\sigma \circ \gamma)
\end{aligned}
$$

on $r_{1}(I)$. By Lemma 5.4, $\cos (\sigma \circ \gamma)=-\sqrt{1-u^{2}}$ on $\Omega$. This entails (ii).

\section{Convexity}

Lemma 7.1 Let $E$ be a bounded open set in $\mathbb{R}^{2}$ with $C^{1,1}$ boundary $M$ and assume that $E \backslash\{0\}=E^{s c}$. Put $d:=\sup \{|x|: x \in M\}>0$ and $b:=(d, 0)$. Let $\gamma_{1}: I \rightarrow M$ be a $C^{1,1}$ parametrisation of $M$ near $b$ with $\gamma_{1}(0)=b$. Then

$$
\lim _{\delta \downarrow 0}\left\{\operatorname{ess}_{[-\delta, \delta]} k_{1}\right\} \geq 1 / d
$$


Proof For $s \in I$,

$$
\gamma_{1}(s)=d e_{1}+s e_{2}+\int_{0}^{s}\left\{\dot{\gamma}_{1}(u)-\dot{\gamma}_{1}(0)\right\} d u
$$

and

$$
\dot{\gamma}_{1}(u)-\dot{\gamma}_{1}(0)=\int_{0}^{u} k_{1} n_{1} d v
$$

by (2.6). By the Fubini-Tonelli Theorem,

$$
\gamma_{1}(s)=d e_{1}+s e_{2}+\int_{0}^{s}(s-u) k_{1}(u) n_{1}(u) d u=d e_{1}+s e_{2}+R(s)
$$

for $s \in I$. Assume for a contradiction that

$$
\lim _{\delta \downarrow 0}\left\{\operatorname{ess} \sup _{[-\delta, \delta]} k_{1}\right\}<l<1 / d
$$

for some $l \in \mathbb{R}$. Then we can find $\delta>0$ such that $k_{1}<l$ a.e. on $[-\delta, \delta]$. So

$$
\left\langle R(s), e_{1}\right\rangle=\int_{0}^{s}(s-u) k_{1}(u)\left\langle n_{1}(u), e_{1}\right\rangle d u>-(1 / 2) s^{2} l(1+o(1))
$$

as $s \downarrow 0$ and

$$
\begin{aligned}
r_{1}(s)^{2}-d^{2} & =2 d\left\langle R(s), e_{1}\right\rangle+s^{2}+o\left(s^{2}\right) \\
& >-d l s^{2}(1+o(1))+s^{2}+o\left(s^{2}\right)
\end{aligned}
$$

as $s \downarrow 0$. Alternatively,

$$
\frac{r_{1}(s)^{2}-d^{2}}{s^{2}}>1-d l+o(1)
$$

As $1-d l>0$ we can find $s \in I$ with $r_{1}(s)>d$, contradicting the definition of $d$.

Lemma 7.2 Let $f$ be as in (1.3) where $h:[0,+\infty) \rightarrow \mathbb{R}$ is a non-decreasing convex function. Given $v>0$ let $E$ be a bounded minimiser of (1.2). Assume that $E$ is open with $C^{1,1}$ boundary $M$ and that $E \backslash\{0\}=E^{s c}$. Suppose that $M \backslash \overline{\Lambda_{1}} \neq \emptyset$. Then $\lambda \leq-1 / d-\rho_{-}(d)<0$ with $\lambda$ as in Theorem 6.5.

Proof We write $M$ as the disjoint union $M=\left(M \backslash \overline{\Lambda_{1}}\right) \cup \overline{\Lambda_{1}}$. Let $b$ be as above. Suppose that $b \in \overline{\Lambda_{1}}$. Then $b \in \Lambda_{1}$; in fact, $b \in \Lambda_{1}^{-}$. By Theorem $6.5, \lambda \leq-\rho_{-}-k$ at $b$. By Lemma 7.1, $\lambda \leq-1 / d-\rho_{-}(d)$ upon considering an appropriate sequence in $M$ converging to $b$. Now suppose that $b$ lies in the open set $M \backslash \overline{\Lambda_{1}}$ in $M$. Let $\gamma_{1}: I \rightarrow M$ be a $C^{1,1}$ parametrisation of $M$ near $b$ with $\gamma_{1}(I) \subset M \backslash \overline{\Lambda_{1}}$. By Theorem $6.5, k_{1}+\rho\left(r_{1}\right) \sin \sigma_{1}+\lambda=0$ a.e. on $I$. Now $\sin \sigma_{1}(s) \rightarrow 1$ as $s \rightarrow 0$. In light of Lemma $7.1,1 / d+\rho(d-)+\lambda \leq 0$ and $\lambda \leq-1 / d-\rho_{-}(d)$. 
Theorem 7.3 Let $f$ be as in (1.3) where $h:[0,+\infty) \rightarrow \mathbb{R}$ is a non-decreasing convex function. Given $v>0$ let $E$ be a bounded minimiser of (1.2). Assume that $E$ is open with $C^{1,1}$ boundary $M$ and that $E \backslash\{0\}=E^{s c}$. Suppose that $M \backslash \overline{\Lambda_{1}} \neq \emptyset$. Then $E$ is convex.

Proof The proof runs along similar lines as [22] Theorem 6.5. By Theorem 6.5, $k+\rho \sin \sigma+\lambda=0 \mathscr{H}^{1}$-a.e. on $M \backslash \overline{\Lambda_{1}}$. By Lemma 7.2,

$$
0 \leq k+\rho_{-}(d)+\lambda \leq k-1 / d
$$

and $k \geq 1 / d \mathscr{H}^{1}$-a.e. on $M \backslash \overline{\Lambda_{1}}$. On $\Lambda_{1}^{+}, k \geq \rho_{-}-\lambda \geq \rho_{-}+\rho_{-}(d)+1 / d>0$; on the other hand, $k<0$ on $\Lambda_{1}^{+}$. So in fact $\Lambda_{1}^{+}=\emptyset$. If $b \in \Lambda_{1}^{-}$then $k=1 / d$. On $\Lambda_{1}^{-} \cap B(0, d), k \geq-\rho_{+}-\lambda \geq-\rho_{+}+\rho_{-}(d)+1 / d \geq 1 / d$. Therefore $k \geq 1 / d>0$ $\mathscr{H}^{1}$-a.e. on $M$. The set $E$ is then convex by a modification of [26] Theorem 1.8 and Proposition 1.4. It is sufficient that the function $f$ (here $\alpha_{1}$ ) in the proof of the former theorem is non-decreasing.

\section{A reverse Hermite-Hadamard inequality}

Let $0 \leq a<b<+\infty$ and $\rho \geq 0$ be a non-decreasing bounded function on $[a, b]$. Let $h$ be a primitive of $\rho$ on $[a, b]$ so that $h \in C^{0,1}([a, b])$ and introduce the functions

$$
\begin{aligned}
& \mathrm{f}:[a, b] \rightarrow \mathbb{R} ; x \mapsto e^{h(x)} ; \\
& g:[a, b] \rightarrow \mathbb{R} ; x \mapsto x \mathrm{f}(x) .
\end{aligned}
$$

Then

$$
g^{\prime}=(1 / x+\rho) g=\mathrm{f}+g \rho
$$

a.e. on $(a, b)$. Define

$$
m=m(\rho, a, b):=\frac{g(b)-g(a)}{\int_{a}^{b} g d t} .
$$

If $\rho$ takes the constant value $\mathbb{R} \ni \lambda \geq 0$ on $[a, b]$ we use the notation $m(\lambda, a, b)$ and we write $m_{0}=m(0, a, b)$. A computation gives

$$
m_{0}=m(0, a, b)=A(a, b)^{-1}
$$

where $A(a, b):=(a+b) / 2$ stands for the arithmetic mean of $a$ and $b$.

Lemma 8.1 Let $0 \leq a<b<+\infty$ and $\rho \geq 0$ be a non-decreasing bounded function on $[a, b]$. Then $m_{0} \leq m$. 
Proof Note that $g$ is convex on $[a, b]$ as can be seen from (8.3). By the HermiteHadamard inequality (cf. $[15,17])$,

$$
\frac{1}{b-a} \int_{a}^{b} g d t \leq \frac{g(a)+g(b)}{2} .
$$

The inequality $(b-a)(g(a)+g(b)) \leq(a+b)(g(b)-g(a))$ entails

$$
\int_{a}^{b} g d t \leq \frac{a+b}{2}(g(b)-g(a))
$$

and the result follows on rearrangement.

Lemma 8.2 Let $0 \leq a<b<+\infty$ and $\lambda>0$. Then $m(\lambda, a, b)<\lambda+A(a, b)^{-1}$.

Proof First suppose that $\lambda=1$ and take $h:[a, b] \rightarrow \mathbb{R} ; t \mapsto t$. In this case,

$$
\int_{a}^{b} g d t=\int_{a}^{b} t e^{t} d t=(b-1) e^{b}-(a-1) e^{a}
$$

and

$$
m(1, a, b)=\frac{b e^{b}-a e^{a}}{(b-1) e^{b}-(a-1) e^{a}} .
$$

The inequality in the statement is equivalent to

$$
(a+b)\left(b e^{b}-a e^{a}\right)<\left((b-1) e^{b}-(a-1) e^{a}\right)(2+a+b)
$$

which in turn is equivalent to the statement $\tanh [(b-a) / 2]<(b-a) / 2$ which holds for any $b>a$.

For $\lambda>0$ take $h:[a, b] \rightarrow \mathbb{R} ; t \mapsto \lambda t$. Substitution gives

$$
\begin{aligned}
\int_{a}^{b} g d t & =(1 / \lambda)^{2}\left[(\lambda b-1) e^{\lambda b}-(\lambda a-1) e^{\lambda a}\right] \text { and } \\
g(b)-g(a) & =(1 / \lambda)\left[\lambda b e^{\lambda b}-\lambda a e^{\lambda a}\right]
\end{aligned}
$$

so from above

$$
m(\lambda, a, b)=\lambda m(1, \lambda a, \lambda b)<\lambda\left\{1+A(\lambda a, \lambda b)^{-1}\right\}=\lambda+A(a, b)^{-1} .
$$

Theorem 8.3 Let $0 \leq a<b<+\infty$ and $\rho \geq 0$ be a non-decreasing bounded function on $[a, b]$. Then

(i) $m(\rho, a, b) \leq \rho(b-)+A(a, b)^{-1}$; 
(ii) equality holds if and only if $\rho \equiv 0$ on $[a, b)$.

Proof (i) Define $h:=\int_{a}^{\cdot} \rho d \tau$ on $[a, b]$ so that $h^{\prime}=\rho$ a.e. on $(a, b)$. Define $h_{1}$ : $[a, b] \rightarrow \mathbb{R} ; t \mapsto h(b)-\rho(b-)(b-t)$. Then $h_{1}(b)=h(b), h_{1}^{\prime}=\rho(b-) \geq \rho=h^{\prime}$ a.e. on $(a, b)$ and hence $h \geq h_{1}$ on $[a, b]$. We derive

$$
\int_{a}^{b} g d t=\int_{a}^{b} t e^{h(t)} d t \geq \int_{a}^{b} t e^{h_{1}(t)} d t=\int_{a}^{b} g_{1} d t
$$

and

$$
\begin{aligned}
g(b)-g(a) & =b e^{h(b)}-a e^{h(a)}=b e^{h_{1}(b)}-a e^{h(a)} \\
& \leq b e^{h_{1}(b)}-a e^{h_{1}(a)}=g_{1}(b)-g_{1}(a)
\end{aligned}
$$

with obvious notation. This entails that $m(\rho, a, b) \leq m(\rho(b-), a, b)$ and the result follows with the help of Lemma 8.2.

(ii) Suppose that $\rho \not \equiv 0$ on $[a, b)$. If $\rho$ is constant on $[a, b]$ the assertion follows from Lemma 8.2. Assume then that $\rho$ is not constant on $[a, b)$. Then $h \not \equiv h_{1}$ on $[a, b]$ in the above notation and $\int_{a}^{b} t e^{h(t)} d t>\int_{a}^{b} t e^{h_{1}(t)} d t$ which entails strict inequality in $(i)$.

With the above notation define

$$
\hat{m}=\hat{m}(\rho, a, b):=\frac{g(a)+g(b)}{\int_{a}^{b} g d t} .
$$

A computation gives

$$
\hat{m}_{0}:=\hat{m}(0, a, b)=\frac{2}{b-a} .
$$

Lemma 8.4 Let $0 \leq a<b<+\infty$ and $\rho \geq 0$ be a non-decreasing bounded function on $[a, b]$. Then $\hat{m} \geq \hat{m}_{0}$.

Proof This follows by the Hermite-Hadamard inequality (8.6).

We prove a reverse Hermite-Hadamard inequality.

Theorem 8.5 Let $0 \leq a<b<+\infty$ and $\rho \geq 0$ be a non-decreasing bounded function on $[a, b]$. Then

(i) $(b-a) \hat{m}(\rho, a, b) \leq 2+a \rho(a+)+b \rho(b-)$;

(ii) equality holds if and only if $\rho \equiv 0$ on $[a, b)$.

This last inequality can be written in the form

$$
\frac{g(a)+g(b)}{2+a \rho(a+)+b \rho(b-)} \leq \frac{1}{b-a} \int_{a}^{b} g d t
$$

comparing with (8.6) justifies naming this a reverse Hermite-Hadamard inequality. 
Proof (i) We assume in the first instance that $\rho \in C^{1}((a, b))$. We prove the above result in the form

$$
\int_{a}^{b} g d t \geq(b-a) \frac{g(a)+g(b)}{2+a \rho(a)+b \rho(b)} .
$$

Put

$$
w:=\frac{(t-a)(g(a)+g)}{2+a \rho(a)+t \rho}
$$

for $t \in[a, b]$ so that

$$
\int_{a}^{b} w^{\prime} d t=(b-a) \frac{g(a)+g(b)}{2+a \rho(a)+b \rho(b)} .
$$

Then using (8.3),

$$
\begin{aligned}
w^{\prime}= & \frac{\left(g(a)+g+(t-a) g^{\prime}\right)(2+a \rho(a)+t \rho)-(t-a)(g(a)+g)\left(\rho+t \rho^{\prime}\right)}{(2+a \rho(a)+t \rho)^{2}} \\
= & \frac{\left(g(a)-a g^{\prime}+(2+t \rho) g\right)(2+a \rho(a)+t \rho)-(t-a)(g(a)+g)\left(\rho+t \rho^{\prime}\right)}{(2+a \rho(a)+t \rho)^{2}} \\
= & \frac{(2+t \rho)(2+a \rho(a)+t \rho)}{(2+a \rho(a)+t \rho)^{2}} g \\
& +\frac{\left(g(a)-a g^{\prime}\right)(2+a \rho(a)+t \rho)-(t-a)(g(a)+g)\left(\rho+t \rho^{\prime}\right)}{(2+a \rho(a)+t \rho)^{2}} \\
\leq & g-\frac{2 g(a)}{(2+a \rho(a)+b \rho(b))^{2}}(t-a) \rho \\
\leq & g
\end{aligned}
$$

on $(a, b)$ as

$$
g(a)-a g^{\prime}=a(\mathrm{f}(a)-(1 / t+\rho) g)=a(\mathrm{f}(a)-\mathrm{f}-\rho g) \leq 0 .
$$

An integration over $[a, b]$ gives the result.

Let us now assume that $\rho \geq 0$ is a non-decreasing bounded function on $[a, b]$. Extend $\rho$ to $\mathbb{R}$ via

$$
\tilde{\rho}(t):= \begin{cases}\rho(a+) & \text { for } t \in(-\infty, a] \\ \rho(t) & \text { for } t \in(a, b] \\ \rho(b-) & \text { for } t \in(b,+\infty)\end{cases}
$$

for $t \in \mathbb{R}$. Let $\left(\psi_{\varepsilon}\right)_{\varepsilon>0}$ be a family of mollifiers (see e.g. [1] 2.1) and set $\widetilde{\rho}_{\varepsilon}:=\widetilde{\rho} \star \psi_{\varepsilon}$ on $\mathbb{R}$ for each $\varepsilon>0$. Then $\widetilde{\rho}_{\varepsilon} \in C^{\infty}(\mathbb{R})$ and is non-decreasing on $\mathbb{R}$ for each $\varepsilon>0$. Put $\rho_{\varepsilon}:=\left.\widetilde{\rho}_{\varepsilon}\right|_{[a, b]}$ for each $\varepsilon>0$. Then $\left(\rho_{\varepsilon}\right)_{\varepsilon>0}$ converges to $\rho$ in $L^{1}((a, b))$ by [1] 
2.1 for example. Note that $h_{\varepsilon}:=\int_{a}^{\cdot} \rho_{\varepsilon} d t \rightarrow h$ pointwise on $[a, b]$ as $\varepsilon \downarrow 0$ and that $\left(h_{\varepsilon}\right)$ is uniformly bounded on $[a, b]$. Moreover, $\rho_{\varepsilon}(a) \rightarrow \rho(a+)$ and $\rho_{\varepsilon}(b) \rightarrow \rho(b-)$ as $\varepsilon \downarrow 0$. By the above result,

$$
(b-a) \hat{m}\left(\rho_{\varepsilon}, a, b\right) \leq 2+a \rho_{\varepsilon}(a)+b \rho_{\varepsilon}(b)
$$

for each $\varepsilon>0$. The inequality follows on taking the limit $\varepsilon \downarrow 0$ with the help of the dominated convergence theorem.

(ii) We now consider the equality case. We claim that

$$
\begin{aligned}
& (b-a) \frac{g(a)+g(b)}{2+a \rho(a+)+b \rho(b-)} \leq \int_{a}^{b} g d t \\
& -\frac{2 g(a)}{(2+a \rho(a+)+b \rho(b-))^{2}} \int_{a}^{b}(t-a) \rho d t
\end{aligned}
$$

this entails the equality condition in (ii). First suppose that $\rho \in C^{1}((a, b))$. In this case the inequality in (8.10) implies (8.11) upon integration. Now suppose that $\rho \geq 0$ is a non-decreasing bounded function on $[a, b]$. Then (8.11) holds with $\rho_{\varepsilon}$ in place of $\rho$ for each $\varepsilon>0$. The inequality for $\rho$ follows by the dominated convergence theorem.

\section{Comparison theorems for first-order differential equations}

Let $\mathscr{L}$ stand for the collection of Lebesgue measurable sets in $[0,+\infty)$. Define a measure $\mu$ on $([0,+\infty), \mathscr{L})$ by $\mu(d x):=(1 / x) d x$. Let $0 \leq a<b<+\infty$. Suppose that $u:[a, b] \rightarrow \mathbb{R}$ is an $\mathscr{L}^{1}$-measurable function with the property that

$$
\mu(\{u>t\})<+\infty \text { for each } t>0 .
$$

The distribution function $\mu_{u}:(0,+\infty) \rightarrow[0,+\infty)$ of $u$ with respect to $\mu$ is given by

$$
\mu_{u}(t):=\mu(\{u>t\}) \text { for } t>0
$$

Note that $\mu_{u}$ is right-continuous and non-increasing on $(0, \infty)$ and $\mu_{u}(t) \rightarrow 0$ as $t \rightarrow \infty$.

Let $u$ be a Lipschitz function on $[a, b]$. Define

$Z_{1}:=\left\{u\right.$ differentiable and $\left.u^{\prime}=0\right\}, Z_{2}:=\{u$ not differentiable $\}$ and $Z:=Z_{1} \cup Z_{2}$.

By [1] Lemma 2.96, $Z \cap\{u=t\}=\emptyset$ for $\mathscr{L}^{1}$-a.e. $t \in \mathbb{R}$ and hence $N:=u(Z) \subset \mathbb{R}$ is $\mathscr{L}^{1}$-negligible. We make use of the coarea formula ( [1] Theorem 2.93 and (2.74)),

$$
\int_{[a, b]} \phi\left|u^{\prime}\right| d x=\int_{-\infty}^{\infty} \int_{\{u=t\}} \phi d \mathscr{H}^{0} d t
$$


for any $\mathscr{L}^{1}$-measurable function $\phi:[a, b] \rightarrow[0, \infty]$.

Lemma 9.1 Let $0 \leq a<b<+\infty$ and $u$ a Lipschitz function on $[a, b]$. Then

(i) $\mu_{u} \in \mathrm{BV}_{\text {loc }}((0,+\infty))$;

(ii) $D \mu_{u}=-u_{\sharp} \mu$;

(iii) $D \mu_{u}^{a}=D \mu_{u}\llcorner((0,+\infty) \backslash N)$;

(iv) $D \mu_{u}^{s}=D \mu_{u}\llcorner N$;

(v) $A:=\left\{t \in(0,+\infty): \mathscr{L}^{1}(Z \cap\{u=t\})>0\right\}$ is the set of atoms of $D \mu_{u}$ and $D \mu_{u}^{j}=D \mu_{u}\llcorner A$;

(vi) $\mu_{u}$ is differentiable $\mathscr{L}^{1}$-a.e. on $(0,+\infty)$ with derivative given by

$$
\mu_{u}^{\prime}(t)=-\int_{\{u=t\} \backslash Z} \frac{1}{\left|u^{\prime}\right|} \frac{d \mathscr{H}^{0}}{\tau}
$$

for $\mathscr{L}^{1}$-a.e. $t \in(0,+\infty)$;

(vii) $\operatorname{Ran}(u) \cap[0,+\infty)=\operatorname{supp}\left(D \mu_{u}\right)$.

The notation above $D \mu_{u}^{a}, D \mu_{u}^{s}, D \mu_{u}^{j}$ stands for the absolutely continuous resp. singular resp. jump part of the measure $D \mu_{u}$ (see [1] 3.2 for example).

Proof For any $\varphi \in C_{c}^{\infty}((0,+\infty))$ with $\operatorname{supp}[\varphi] \subset(\tau,+\infty)$ for some $\tau>0$,

$$
\begin{aligned}
\int_{0}^{\infty} \mu_{u} \varphi^{\prime} d t & =\int_{[a, b]} \varphi \circ u d \mu \\
& =\int_{[a, b]} \chi_{\{u>\tau\}} \varphi \circ u d \mu
\end{aligned}
$$

by Fubini's theorem; so $\mu_{u} \in \mathrm{BV}_{\text {loc }}((0,+\infty))$ and $D \mu_{u}$ is the push-forward of $\mu$ under $u, D \mu_{u}=-u_{\sharp} \mu$ (cf. [1] 1.70). By (9.2),

$$
\begin{aligned}
D \mu_{u}\llcorner((0,+\infty) \backslash N)(A) & =-\mu(\{u \in A\} \backslash Z) \\
& =-\int_{A} \int_{\{u=t\} \backslash Z} \frac{1}{\left|u^{\prime}\right|} \frac{d \mathcal{H}^{0}}{\tau} d t
\end{aligned}
$$

for any $\mathscr{L}^{1}$-measurable set $A$ in $(0,+\infty)$. In light of the above, we may identify $D \mu_{u}^{a}=D \mu_{u}\left\llcorner((0,+\infty) \backslash N)\right.$ and $D \mu_{u}^{s}=D \mu_{u}\left\llcorner N\right.$. The set of atoms of $D \mu_{u}$ is defined by $A:=\left\{t \in(0,+\infty): D \mu_{u}(\{t\}) \neq 0\right\}$. For $t>0$,

$$
\begin{aligned}
D \mu_{u}(\{t\}) & =D \mu_{u}^{s}(\{t\})=\left(D \mu_{u}\llcorner N)((\{t\})\right. \\
& =-u_{\sharp} \mu(N \cap\{t\})=-\mu(Z \cap\{u=t\})
\end{aligned}
$$

and this entails $(v)$. The monotone function $\mu_{u}$ is a good representative within its equivalence class and is differentiable $\mathscr{L}^{1}$-a.e. on $(0,+\infty)$ with derivative given by the density of $D \mu_{u}$ with respect to $\mathscr{L}^{1}$ by [1] Theorem 3.28. Item (vi) follows from (9.2) and (iii). Item (vii) follows from (ii). 
Let $0<a<b<+\infty$ and $\rho \geq 0$ be a non-decreasing bounded function on $[a, b]$. Let $\eta \in\{ \pm 1\}^{2}$. We study solutions to the first-order linear ordinary differential equation

$$
u^{\prime}+(1 / x+\rho) u+\lambda=0 \text { a.e. on }(a, b) \text { with } u(a)=\eta_{1} \text { and } u(b)=\eta_{2}
$$

where $u \in C^{0,1}([a, b])$ and $\lambda \in \mathbb{R}$. In case $\rho \equiv 0$ on $[a, b]$ we use the notation $u_{0}$.

Lemma 9.2 Let $0<a<b<+\infty$ and $\rho \geq 0$ be a non-decreasing bounded function on $[a, b]$. Let $\eta \in\{ \pm 1\}^{2}$. Then

(i) there exists a solution $(u, \lambda)$ of (9.4) with $u \in C^{0,1}([a, b])$ and $\lambda=\lambda_{\eta} \in \mathbb{R}$;

(ii) the pair $(u, \lambda)$ in (i) is unique;

(iii) $\lambda_{\eta}$ is given by

$$
-\lambda_{(1,1)}=\lambda_{(-1,-1)}=m ; \lambda_{(1,-1)}=-\lambda_{(-1,1)}=\hat{m}
$$

(iv) if $\eta=(1,1)$ or $\eta=(-1,-1)$ then $u$ is uniformly bounded away from zero on $[a, b]$.

Proof (i) For $\eta=(1,1)$ define $u:[a, b] \rightarrow \mathbb{R}$ by

$$
u(t):=\frac{m \int_{a}^{t} g d s+g(a)}{g(t)} \text { for } t \in[a, b]
$$

with $m$ as in (8.4). Then $u \in C^{0,1}([a, b])$ and satisfies (9.4) with $\lambda=-m$. For $\eta=(1,-1)$ set $u=\left(-\hat{m} \int_{a}^{*} g d s+g(a)\right) / g$ with $\lambda=\hat{m}$. The cases $\eta=(-1,-1)$ and $\eta=(-1,1)$ can be dealt with using linearity. ( $i i)$ We consider the case $\eta=(1,1)$. Suppose that $\left(u_{1}, \lambda_{1}\right)$ resp. $\left(u_{2}, \lambda_{2}\right)$ solve (9.4). By linearity $u:=u_{1}-u_{2}$ solves

$$
u^{\prime}+(1 / x+\rho) u+\lambda=0 \text { a.e. on }(a, b) \text { with } u(a)=u(b)=0
$$

where $\lambda=\lambda_{1}-\lambda_{2}$. An integration gives that $u=\left(-\lambda \int_{a}^{\cdot} g d s+c\right) / g$ for some constant $c \in \mathbb{R}$ and the boundary conditions entail that $\lambda=c=0$. The other cases are similar. (iii) follows as in (i). (iv) If $\eta=(1,1)$ then $u>0$ on $[a, b]$ from (9.5) as $m>0$.

The boundary condition $\eta_{1} \eta_{2}=-1$.

Lemma 9.3 Let $0<a<b<+\infty$ and $\rho \geq 0$ be a non-decreasing bounded function on $[a, b]$. Let $(u, \lambda)$ solve (9.4) with $\eta=(1,-1)$. Then

(i) there exists a unique $c \in(a, b)$ with $u(c)=0$;

(ii) $u^{\prime}<0$ a.e. on $[a, c]$ and $u$ is strictly decreasing on $[a, c]$;

(iii) $D \mu_{u}^{s}=0$. 
Proof (i) We first observe that $u^{\prime} \leq-\hat{m}<0$ a.e. on $\{u \geq 0\}$ in view of (9.4). Suppose $u\left(c_{1}\right)=u\left(c_{2}\right)=0$ for some $c_{1}, c_{2} \in(a, b)$ with $c_{1}<c_{2}$. We may assume that $u \geq 0$ on $\left[c_{1}, c_{2}\right]$. This contradicts the above observation. Item (ii) is plain. For any $\mathscr{L}^{\overline{1}}$-measurable set $B$ in $(0,+\infty), D \mu_{u}^{s}(B)=\mu(\{u \in B\} \cap Z)=0$ using Lemma 9.1 and (ii).

Lemma 9.4 Let $0<a<b<+\infty$ and $\rho \geq 0$ be a non-decreasing bounded function on $[a, b]$. Let $(u, \lambda)$ solve (9.4) with $\eta=(1,-1)$. Assume that

(a) $u$ is differentiable at both $a$ and $b$ and that (9.4) holds there;

(b) $u^{\prime}(a)<0$ and $u^{\prime}(b)<0$;

(c) $\rho$ is differentiable at $a$ and $b$.

Put $v:=-u$. Then

(i) $\int_{\{v=1\} \backslash Z_{v}} \frac{1}{\left|v^{\prime}\right|} \frac{d \mathscr{H}^{0}}{\tau} \geq \int_{\{u=1\} \backslash Z_{u}} \frac{1}{\left|u^{\prime}\right|} \frac{d \mathscr{H}^{0}}{\tau}$;

(ii) equality holds if and only if $\rho \equiv 0$ on $[a, b)$.

Proof First, $\{u=1\}=\{a\}$ by Lemma 9.3. Further $0<-a u^{\prime}(a)=1+a[\hat{m}+\rho(a)]$ from (9.4). On the other hand $\{v=1\} \supset\{b\}$ and $0<b v^{\prime}(b)=-1+b[\hat{m}-\rho(b)]$. Thus

$$
\begin{aligned}
& \int_{\{v=1\} \backslash Z_{v}} \frac{1}{\left|v^{\prime}\right|} \frac{d \mathscr{H}^{0}}{\tau}-\int_{\{u=1\} \backslash Z_{u}} \frac{1}{\left|u^{\prime}\right|} \frac{d \mathscr{H}^{0}}{\tau} \\
& \geq \frac{1}{-1+b[\hat{m}-\rho(b)]}-\frac{1}{1+a[\hat{m}+\rho(a)]} .
\end{aligned}
$$

By Theorem 8.5, $0 \leq 2+(a-b) \hat{m}+a \rho(a)+b \rho(b)$, noting that $\rho(a)=\rho(a+)$ in virtue of (c) and similarly at $b$. A rearrangement leads to the inequality. The equality assertion follows from Theorem 8.5.

Theorem 9.5 Let $0<a<b<+\infty$ and $\rho \geq 0$ be a non-decreasing bounded function on $[a, b]$. Suppose that $(u, \lambda)$ solves $(9.4)$ with $\eta=(1,-1)$ and set $v:=-u$. Assume that $u>-1$ on $[a, b)$. Then

(i) $-\mu_{v}^{\prime} \geq-\mu_{u}^{\prime}$ for $\mathscr{L}^{1}$-a.e. $t \in(0,1)$;

(ii) if $\rho \neq \equiv 0$ on $[a, b)$ then there exists $t_{0} \in(0,1)$ such that $-\mu_{v}^{\prime}>-\mu_{u}^{\prime}$ for $\mathscr{L}^{1}$-a.e. $t \in\left(t_{0}, 1\right)$;

(iii) for $t \in[-1,1]$,

$$
\mu_{u_{0}}(t)=\log \left\{\frac{-(b-a) t+\sqrt{(b-a)^{2} t^{2}+4 a b}}{2 a}\right\}
$$

and $\mu_{v_{0}}=\mu_{u_{0}}$ on $[-1,1]$;

in obvious notation.

Proof (i) The set

$Y_{u}:=Z_{2, u} \cup\left(\left\{u^{\prime}+(1 / x+\rho) u+\lambda \neq 0\right\} \backslash Z_{2, u}\right) \cup\{\rho$ not differentiable $\} \subset[a, b]$ 
(in obvious notation) is a null set in $[a, b]$ and likewise for $Y_{v}$. By [1] Lemma 2.95 and Lemma 2.96, $\{u=t\} \cap\left(Y_{u} \cup Z_{1, u}\right)=\emptyset$ for a.e. $t \in(0,1)$ and likewise for the function $v$. Let $t \in(0,1)$ and assume that $\{u=t\} \cap\left(Y_{u} \cup Z_{1, u}\right)=\emptyset$ and $\{v=t\} \cap\left(Y_{v} \cup Z_{1, v}\right)=$ $\emptyset$. Put $c:=\max \{u \geq t\}$. Then $c \in(a, b),\{u>t\}=[a, c)$ by Lemma 9.3 and $u$ is differentiable at $c$ with $u^{\prime}(c)<0$. Put $d:=\max \{v \leq t\}=\max \{u \geq-t\}$. As $u$ is continuous on $[a, b]$ it holds that $a<c<d<b$. Moreover, $u^{\prime}(d)<0$ as $v(d)=t$ and $d \notin Z_{v}$. Put $\tilde{u}:=u / t$ and $\tilde{v}:=v / t$ on $[c, d]$. Then

$$
\begin{aligned}
& \widetilde{u}^{\prime}+(1 / \tau+\rho) \widetilde{u}+\hat{m} / t=0 \text { a.e. on }(c, d) \text { and } \widetilde{u}(c)=-\widetilde{u}(d)=1 ; \\
& \widetilde{v}^{\prime}+(1 / \tau+\rho) \widetilde{v}-\hat{m} / t=0 \text { a.e. on }(c, d) \text { and }-\widetilde{v}(c)=\widetilde{v}(d)=1 .
\end{aligned}
$$

By Lemma 9.4,

$$
\begin{aligned}
\int_{\{v=t\} \backslash Z_{v}} \frac{1}{\left|v^{\prime}\right|} \frac{d \mathscr{H}^{0}}{\tau} & \geq \int_{[c, d] \cap\{v=t\} \backslash Z_{v}} \frac{1}{\left|v^{\prime}\right|} \frac{d \mathscr{H}^{0}}{\tau} \\
& =(1 / t) \int_{[c, d] \cap\{\tilde{v}=1\} \backslash Z_{v}} \frac{1}{\left|\widetilde{v}^{\prime}\right|} \frac{d \mathscr{H}^{0}}{\tau} \\
& \geq(1 / t) \int_{[c, d] \cap\{\tilde{u}=1\} \backslash Z_{u}} \frac{1}{\left|\widetilde{u}^{\prime}\right|} \frac{d \mathscr{H}^{0}}{\tau} \\
& =\int_{\{u=t\} \backslash Z_{u}} \frac{1}{\left|u^{\prime}\right|} \frac{d \mathscr{H}^{0}}{\tau} .
\end{aligned}
$$

By Lemma 9.1,

$$
-\mu_{u}^{\prime}(t)=\int_{\{u=t\} \backslash Z_{u}} \frac{1}{\left|u^{\prime}\right|} \frac{d \mathscr{H}^{0}}{\tau}
$$

for $\mathscr{L}^{1}$-a.e. $t \in(0,1)$ and a similar formula holds for $v$. The assertion in $(i)$ follows. (ii) Assume that $\rho \not \equiv 0$ on $[a, b)$. Put $\alpha:=\inf \{\rho>0\} \in[a, b)$. Note that $\max \{v \leq$ $t\} \rightarrow b$ as $t \uparrow 1$ as $v<1$ on $[a, b)$ by assumption. Choose $t_{0} \in(0,1)$ such that $\max \left\{v \leq t_{0}\right\}>\alpha$. Then for $t>t_{0}$,

$$
a<\max \{u \geq t\}<\max \left\{u \geq-t_{0}\right\}=\max \left\{v \leq t_{0}\right\}<\max \{v \leq t\}<d
$$

that is, the interval $[c, d]$ with $c, d$ as described above intersects $(\alpha, b]$. So for $\mathscr{L}^{1}$-a.e. $t \in\left(t_{0}, 1\right)$,

$$
\int_{\{v=t\} \backslash Z_{v}} \frac{1}{\left|v^{\prime}\right|} \frac{d \mathscr{H}^{0}}{\tau}>\int_{\{u=t\} \backslash Z_{u}} \frac{1}{\left|u^{\prime}\right|} \frac{d \mathscr{H}^{0}}{\tau} .
$$

by the equality condition in Lemma 9.4. The conclusion follows from the representation of $\mu_{u}$ resp. $\mu_{v}$ in Lemma 9.1. 
(iii) A direct computation gives

$$
u_{0}(\tau)=\frac{1}{b-a}\left\{-\tau+\frac{a b}{\tau}\right\}
$$

for $\tau \in[a, b] ; u_{0}$ is strictly decreasing on its domain. This leads to the formula in (iii). A similar computation gives

$$
\mu_{v_{0}}(t)=\log \left\{\frac{2 b}{(b-a) t+\sqrt{(b-a)^{2} t^{2}+4 a b}}\right\}
$$

for $t \in[-1,1]$. Rationalising the denominator results in the stated equality.

Corollary 9.6 Let $0<a<b<+\infty$ and $\rho \geq 0$ be a non-decreasing bounded function on $[a, b]$. Suppose that $(u, \lambda)$ solves $(9.4)$ with $\eta=(1,-1)$ and set $v:=-u$. Assume that $u>-1$ on $[a, b)$. Then

(i) $\mu_{u}(t) \leq \mu_{v}(t)$ for each $t \in(0,1)$;

(ii) if $\rho \neq \equiv$ on $[a, b)$ then $\mu_{u}(t)<\mu_{v}(t)$ for each $t \in(0,1)$.

Proof (i) By [1] Theorem 3.28 and Lemma 9.3,

$$
\begin{aligned}
\mu_{u}(t) & =\mu_{u}(t)-\mu_{u}(1)=-D \mu_{u}((t, 1]) \\
& =-D \mu_{u}^{a}((t, 1])-D \mu_{u}^{s}((t, 1]) \\
& =-\int_{(t, 1]} \mu_{u}^{\prime} d s
\end{aligned}
$$

for each $t \in(0,1)$ as $\mu_{u}(1)=0$. On the other hand,

$$
\begin{aligned}
\mu_{v}(t) & =\mu_{v}(1)+\left(\mu_{v}(t)-\mu_{v}(1)\right)=\mu_{v}(1)-D \mu_{v}((t, 1]) \\
& =\mu_{v}(1)-\int_{(t, 1]} \mu_{v}^{\prime} d s-D \mu_{v}^{s}((t, 1])
\end{aligned}
$$

for each $t \in(0,1)$. The claim follows from Theorem 9.5 noting that $D \mu_{v}^{s}((t, 1]) \leq 0$ as can be seen from Lemma 9.1. Item (ii) follows from Theorem 9.5 (ii).

Corollary 9.7 Let $0<a<b<+\infty$ and $\rho \geq 0$ be a non-decreasing bounded function on $[a, b]$. Suppose that $(u, \lambda)$ solves $(9.4)$ with $\eta=(1,-1)$. Assume that $u>-1$ on $[a, b)$. Let $\varphi \in C^{1}((-1,1))$ be an odd strictly increasing function with $\varphi \in L^{1}((-1,1))$. Then

(i) $\int_{\{u>0\}} \varphi(u) d \mu<+\infty$;

(ii) $\int_{a}^{b} \varphi(u) d \mu \leq 0$;

(iii) equality holds in (ii) if and only if $\rho \equiv 0$ on $[a, b)$.

In particular,

(iv) $\int_{a}^{b} \frac{u}{\sqrt{1-u^{2}}} d \mu \leq 0$ with equality if and only if $\rho \equiv 0$ on $[a, b)$. 
Proof (i) Put $I:=\{1>u>0\}$. The function $u: I \rightarrow(0,1)$ is $C^{0,1}$ and $u^{\prime} \leq-\hat{m}$ a.e. on $I$ by Lemma 9.3. It has $C^{0,1}$ inverse $v:(0,1) \rightarrow I, v^{\prime}=1 /\left(u^{\prime} \circ v\right)$ and $\left|v^{\prime}\right| \leq 1 / \hat{m}$ a.e. on $(0,1)$. By a change of variables,

$$
\int_{\{u>0\}} \varphi(u) d \mu=\int_{0}^{1} \varphi\left(v^{\prime} / v\right) d t
$$

from which the claim is apparent. (ii) The integral is well-defined because $\varphi(u)^{+}=$ $\varphi(u) \chi_{\{u>0\}} \in L^{1}((a, b), \mu)$ by $(i)$. By Lemma 9.3 the set $\{u=0\}$ consists of a singleton and has $\mu$-measure zero. So

$$
\begin{aligned}
\int_{a}^{b} \varphi(u) d \mu & =\int_{\{u>0\}} \varphi(u) d \mu+\int_{\{u<0\}} \varphi(u) d \mu \\
& =\int_{\{u>0\}} \varphi(u) d \mu-\int_{\{v>0\}} \varphi(v) d \mu
\end{aligned}
$$

where $v:=-u$ as $\varphi$ is an odd function. We remark that in a similar way to (9.3),

$$
\begin{aligned}
\int_{0}^{1} \varphi^{\prime} \mu_{u} d t & =\int_{\{u>0\}}\{\varphi(u)-\varphi(0)\} \\
d \mu & =\int_{\{u>0\}} \varphi(u) d \mu
\end{aligned}
$$

using oddness of $\varphi$ and an analogous formula holds with $v$ in place of $u$. Thus we may write

$$
\begin{aligned}
\int_{a}^{b} \varphi(u) d \mu & =\int_{0}^{1} \varphi^{\prime} \mu_{u} d t-\int_{0}^{1} \varphi^{\prime} \mu_{v} d t \\
& =\int_{0}^{1} \varphi^{\prime}\left\{\mu_{u}-\mu_{v}\right\} d t \leq 0
\end{aligned}
$$

by Corollary 9.6 as $\varphi^{\prime}>0$ on $(0,1)$. (iii) Suppose that $\rho \not \equiv 0$ on $[a, b)$. Then strict inequality holds in the above by Corollary 9.6. If $\rho \equiv 0$ on $[a, b)$ the equality follows from Theorem 9.5. (iv) follows from (ii) and (iii) with the particular choice $\varphi:(-1,1) \rightarrow \mathbb{R} ; t \mapsto t / \sqrt{1-t^{2}}$.

The boundary condition $\eta_{1} \eta_{2}=1$. Let $0<a<b<+\infty$ and $\rho \geq 0$ be a nondecreasing bounded function on $[a, b]$. We study solutions of the auxilliary Riccati equation

$$
w^{\prime}+\lambda w^{2}=(1 / x+\rho) w \text { a.e. on }(a, b) \text { with } w(a)=w(b)=1
$$

with $w \in C^{0,1}([a, b])$ and $\lambda \in \mathbb{R}$. If $\rho \equiv 0$ on $[a, b]$ then we write $w_{0}$ instead of $w$. Suppose $(u, \lambda)$ solves $(9.4)$ with $\eta=(1,1)$. Then $u>0$ on $[a, b]$ by Lemma 9.2 and we may set $w:=1 / u$. Then $(w,-\lambda)$ satisfies (9.6). 
Lemma 9.8 Let $0<a<b<+\infty$ and $\rho \geq 0$ be a non-decreasing bounded function on $[a, b]$. Then

(i) there exists a solution $(w, \lambda)$ of $(9.6)$ with $w \in C^{0,1}([a, b])$ and $\lambda \in \mathbb{R}$;

(ii) the pair $(w, \lambda)$ in (i) is unique;

(iii) $\lambda=m$.

Proof (i) Define $w:[a, b] \rightarrow \mathbb{R}$ by

$$
w(t):=\frac{g(t)}{m \int_{a}^{t} g d s+g(a)} \text { for } t \in[a, b] .
$$

Then $w \in C^{0,1}([a, b])$ and $(w, m)$ satisfies (9.6). (ii) We claim that $w>0$ on $[a, b]$ for any solution $(w, \lambda)$ of (9.6). For otherwise, $c:=\min \{w=0\} \in(a, b)$. Then $u:=1 / w$ on $[a, c)$ satisfies

$$
u^{\prime}+\left(\frac{1}{\tau}+\rho\right) u-\lambda=0 \text { a.e. on }(a, c) \text { and } u(a)=1, u(c-)=+\infty \text {. }
$$

Integrating, we obtain

$$
g u-g(a)-\lambda \int_{a}^{\cdot} g d t=0 \text { on }[a, c)
$$

and this entails the contradiction that $u(c-)<+\infty$. We may now use the uniqueness statement in Lemma 9.2. (iii) follows from (ii) and the particular solution given in $(i)$.

We introduce the mapping

$$
\omega:(0, \infty) \times(0, \infty) \rightarrow \mathbb{R} ;(t, x) \mapsto-(2 / t) \operatorname{coth}(x / 2) .
$$

For $\xi>0$,

$$
|\omega(t, x)-\omega(t, y)| \leq \operatorname{cosech}^{2}[\xi / 2](1 / t)|x-y|
$$

for $(t, x),(t, y) \in(0, \infty) \times(\xi, \infty)$ and $\omega$ is locally Lipschitzian in $x$ on $(0, \infty) \times$ $(0, \infty)$ in the sense of [16] I.3. Let $0<a<b<+\infty$ and set $\lambda:=A / G>1$. Here, $A=A(a, b)$ stands for the arithmetic mean of $a, b$ as introduced in the previous Section while $G=G(a, b):=\sqrt{|a b|}$ stands for their geometric mean. We refer to the inital value problem

$$
z^{\prime}=\omega(t, z) \text { on }(0, \lambda) \text { and } z(1)=\mu((a, b))
$$

Define

$$
z_{0}:(0, \lambda) \rightarrow \mathbb{R} ; t \mapsto 2 \log \left\{\frac{\lambda+\sqrt{\lambda^{2}-t^{2}}}{t}\right\}
$$


Lemma 9.9 Let $0<a<b<+\infty$. Then

(i) $w_{0}(\tau)=\frac{2 A \tau}{G^{2}+\tau^{2}}$ for $\tau \in[a, b]$;

(ii) $\left\|w_{0}\right\|_{\infty}=\lambda$;

(iii) $\mu_{w_{0}}=z_{0}$ on $[1, \lambda)$;

(iv) $z_{0}$ satisfies (9.8) and this solution is unique;

(v) $\int_{\left\{w_{0}=1\right\}} \frac{1}{\left|w_{0}^{\prime}\right|} \frac{d \mathscr{H}^{0}}{\tau}=2 \operatorname{coth}(\mu((a, b)) / 2)$;

(vi) $\int_{a}^{b} \frac{1}{\sqrt{w_{0}^{2}-1}} \frac{d x}{x}=\pi$.

Proof ( $i$ ) follows as in the proof of Lemma 9.8 with $g(t)=t$ while (ii) follows by calculus. (iii) follows by solving the quadratic equation $t \tau^{2}-2 A \tau+G^{2} t=0$ for $\tau$ with $t \in(0, \lambda)$. Uniqueness in (iv) follows from [16] Theorem 3.1 as $\omega$ is locally Lipschitzian with respect to $x$ in $(0, \infty) \times(0, \infty)$. For $(v)$ note that $\left|a w_{0}^{\prime}(a)\right|=1-a / A$ and $\left|b w_{0}^{\prime}(b)\right|=b / A-1$ and

$$
2 \operatorname{coth}(\mu((a, b)) / 2)=2(a+b) /(b-a) .
$$

(vi) We may write

$$
\begin{aligned}
\int_{a}^{b} \frac{1}{\sqrt{w_{0}^{2}-1}} \frac{d \tau}{\tau} & =\int_{a}^{b} \frac{a b+\tau^{2}}{\sqrt{(a+b)^{2} \tau^{2}-\left(a b+\tau^{2}\right)^{2}}} \frac{d \tau}{\tau} \\
& =\int_{a}^{b} \frac{a b+\tau^{2}}{\sqrt{\left(\tau^{2}-a^{2}\right)\left(b^{2}-\tau^{2}\right)}} \frac{d \tau}{\tau}
\end{aligned}
$$

The substitution $s=\tau^{2}$ followed by the Euler substitution (cf. [14] 2.251)

$$
\sqrt{\left(s-a^{2}\right)\left(b^{2}-s\right)}=t\left(s-a^{2}\right)
$$

gives

$$
\int_{a}^{b} \frac{1}{\sqrt{w_{0}^{2}-1}} \frac{d \tau}{\tau}=\int_{0}^{\infty} \frac{1}{1+t^{2}}+\frac{a b}{b^{2}+a^{2} t^{2}} d t=\pi
$$

Lemma 9.10 Let $0<a<b<+\infty$. Then

(i) for $y>$ a the function $x \mapsto \frac{b y-a x}{(y-a)(b-x)}$ is strictly increasing on $(-\infty, b]$;

(ii) the function $y \mapsto \frac{(b-a) y}{(y-a)(b-y)}$ is strictly increasing on $[G, b]$;

(iii) for $x<b$ the function $y \mapsto \frac{b y-a x}{(y-a)(b-x)}$ is strictly decreasing on $[a,+\infty)$

Proof The proof is an exercise in calculus.

Lemma 9.11 Let $0<a<b<+\infty$ and $\rho \geq 0$ be a non-decreasing bounded function on $[a, b]$. Let $(w, \lambda)$ solve (9.6). Assume 
(i) $w$ is differentiable at both $a$ and $b$ and that (9.6) holds there;

(ii) $w^{\prime}(a)>0$ and $w^{\prime}(b)<0$;

(iii) $w>1$ on $(a, b)$;

(iv) $\rho$ is differentiable at $a$ and $b$.

Then

$$
\int_{\{w=1\} \backslash Z_{w}} \frac{1}{\left|w^{\prime}\right|} \frac{d \mathscr{H}^{0}}{\tau} \geq 2 \operatorname{coth}(\mu((a, b)) / 2)
$$

with equality if and only if $\rho \equiv 0$ on $[a, b)$.

Proof At the end-points $x=a, b$ the condition ( $i$ ) entails that $w^{\prime}+m-\rho=1 / x=$ $w_{0}^{\prime}+m_{0}$ so that

$$
w^{\prime}-w_{0}^{\prime}=m_{0}-m+\rho \text { at } x=a, b .
$$

We consider the four cases

(a) $w^{\prime}(a) \geq w_{0}^{\prime}(a)$ and $w^{\prime}(b) \geq w_{0}^{\prime}(b)$;

(b) $w^{\prime}(a) \geq w_{0}^{\prime}(a)$ and $w^{\prime}(b) \leq w_{0}^{\prime}(b)$;

(c) $w^{\prime}(a) \leq w_{0}^{\prime}(a)$ and $w^{\prime}(b) \geq w_{0}^{\prime}(b)$;

(d) $w^{\prime}(a) \leq w_{0}^{\prime}(a)$ and $w^{\prime}(b) \leq w_{0}^{\prime}(b)$;

in turn.

(a) Condition (a) together with (9.9) means that $m_{0}-m+\rho(a) \geq 0$; that is, $m-$ $\rho(a) \leq m_{0}$. By $(i)$ and $(i i), b m-b \rho(b)-1=-b w^{\prime}(b)>0$; or $m-\rho(b)>1 / b$. Therefore,

$$
0<1 / b<m-\rho(b) \leq m-\rho(a) \leq 1 / A
$$

by (8.5). Put $x:=1 /(m-\rho(b))$ and $y:=1 /(m-\rho(a))$. Then

$$
a<A \leq y \leq x<b .
$$

We write

$$
\begin{gathered}
a w^{\prime}(a)=-(m-\rho(a)) a+1=-(1 / y) a+1>0 \\
b w^{\prime}(b)=-(m-\rho(b)) b+1=-(1 / x) b+1<0 .
\end{gathered}
$$

Making use of assumption (iii),

$$
\begin{aligned}
\int_{\{w=1\} \backslash Z_{w}} \frac{1}{\left|w^{\prime}\right|} \frac{d \mathscr{H}^{0}}{x} & =\frac{1}{-(1 / y) a+1}-\frac{1}{-(1 / x) b+1} \\
& =\frac{b y-a x}{(y-a)(b-x)} .
\end{aligned}
$$


By Lemma 9.10 (i) then (ii),

$$
\begin{aligned}
\int_{\{w=1\}} \frac{1}{\left|w^{\prime}\right|} \frac{d \mathscr{H}^{0}}{x} & \geq \frac{(b-a) y}{(y-a)(b-y)} \geq \frac{(b-a) A}{(A-a)(b-A)} \\
& =2 \frac{a+b}{b-a}=2 \operatorname{coth}(\mu((a, b)) / 2) .
\end{aligned}
$$

If equality holds then $\rho(a)=\rho(b)$ and $\rho$ is constant on $[a, b]$. By Theorem 8.3 we conclude that $\rho \equiv 0$ on $[a, b)$.

(b) Condition (b) together with (9.9) entails that $0 \leq m_{0}-m+\rho$ (a) and $0 \leq$ $-m_{0}+m-\rho(b)$ whence $0 \leq \rho(a)-\rho(b)$ upon adding; so $\rho$ is constant on the interval $[a, b]$ by monotonicity. Define $x$ and $y$ as above. Then $x=y$ and $y \geq A$. The result now follows in a similar way to case (a).

(c) In this case,

$$
\begin{aligned}
\frac{1}{a w^{\prime}(a)}-\frac{1}{b w^{\prime}(b)} & \geq \frac{1}{a w_{0}^{\prime}(a)}-\frac{1}{b w_{0}^{\prime}(b)} \\
& =2 \operatorname{coth}(\mu((a, b)) / 2)
\end{aligned}
$$

by Lemma 9.9. If equality holds then $w^{\prime}(b)=w_{0}^{\prime}(b)$ so that $m_{0}-m+\rho(b)=0$ and $\rho$ vanishes on $[a, b]$ by Theorem 8.3.

(d) Condition (d) together with (9.9) means that $m_{0}-m+\rho(b) \leq 0$; that is, $m \geq$ $\rho(b)+m_{0}$. On the other hand, by Theorem 8.3, $m \leq \rho(b)+m_{0}$. In consequence, $m=\rho(b)+m_{0}$. It then follows that $\rho \equiv 0$ on $[a, b]$ by Theorem 8.3. Now use Lemma 9.9.

Lemma 9.12 Let $\phi:(0,+\infty) \rightarrow(0,+\infty)$ be a convex non-increasing function with $\inf _{(0,+\infty)} \phi>0$. Let $\Lambda$ be an at most countably infinite index set and $\left(x_{h}\right)_{h \in \Lambda} a$ sequence of points in $(0,+\infty)$ with $\sum_{h \in \Lambda} x_{h}<+\infty$. Then

$$
\sum_{h \in \Lambda} \phi\left(x_{h}\right) \geq \phi\left(\sum_{h \in \Lambda} x_{h}\right)
$$

and the left-hand side takes the value $+\infty$ in case $\Lambda$ is countably infinite and is otherwise finite.

Proof Suppose $0<x_{1}<x_{2}<+\infty$. By convexity $\phi\left(x_{1}\right)+\phi\left(x_{2}\right) \geq 2 \phi\left(\frac{x_{1}+x_{2}}{2}\right) \geq$ $\phi\left(x_{1}+x_{2}\right)$ as $\phi$ is non-increasing. The result for finite $\Lambda$ follows by induction.

Theorem 9.13 Let $0<a<b<+\infty$ and $\rho \geq 0$ be a non-decreasing bounded function on $[a, b]$. Let $(w, \lambda)$ solve (9.6). Assume that $w>1$ on $(a, b)$. Then

(i) for $\mathscr{L}^{1}$-a.e. $t \in\left(1,\|w\|_{\infty}\right)$,

$$
-\mu_{w}^{\prime} \geq(2 / t) \operatorname{coth}\left((1 / 2) \mu_{w}\right)
$$


(ii) if $\rho \not \equiv 0$ on $[a, b)$ then there exists $t_{0} \in\left(1,\|w\|_{\infty}\right)$ such that strict inequality holds in (9.10) for $\mathscr{L}^{1}$-a.e. $t \in\left(1, t_{0}\right)$.

Proof (i) The set

$$
\begin{aligned}
Y_{w}:= & Z_{2, w} \cup\left(\left\{w^{\prime}+m w^{2} \neq(1 / x+\rho) w\right\} \backslash Z_{2, w}\right) \\
& \cup\{\rho \text { not differentiable }\} \subset[a, b]
\end{aligned}
$$

is a null set in $[a, b]$. By [1] Lemma 2.95 and Lemma 2.96, $\{w=t\} \cap\left(Y_{w} \cap Z_{1, w}\right)=\emptyset$ for a.e. $t>1$. Let $t \in\left(1,\|w\|_{\infty}\right)$ and assume that $\{w=t\} \cap\left(Y_{w} \cap Z_{1, w}\right)=\emptyset$. We write $\{w>t\}=\bigcup_{h \in \Lambda} I_{h}$ where $\Lambda$ is an at most countably infinite index set and $\left(I_{h}\right)_{h \in \Lambda}$ are disjoint non-empty well-separated open intervals in $(a, b)$. The term well-separated means that for each $h \in \Lambda, \inf _{k \in \Lambda \backslash\{h\}} d\left(I_{h}, I_{k}\right)>0$. This follows from the fact that $w^{\prime} \neq 0$ on $\partial I_{h}$ for each $h \in \Lambda$. Put $\widetilde{w}:=w / t$ on $\overline{\{w>t\}}$ so

$$
\widetilde{w}^{\prime}+(m t) \widetilde{w}^{2}=(1 / x+\rho) \widetilde{w} \text { a.e. on }\{w>t\} \text { and } \widetilde{w}=1 \text { on }\{w=t\}
$$

We use the fact that the mapping $\phi:(0,+\infty) \rightarrow(0,+\infty) ; t \mapsto \operatorname{coth} t$ satisfies the hypotheses of Lemma 9.12. By Lemmas 9.11 and 9.12,

$$
\begin{aligned}
(0,+\infty] \ni \int_{\{w=t\} \backslash Z_{w}} \frac{1}{\left|w^{\prime}\right|} \frac{d \mathscr{H}^{0}}{x} & =(1 / t) \int_{\{\widetilde{w}=1\}} \frac{1}{\left|\widetilde{w}^{\prime}\right|} \frac{d \mathscr{H}^{0}}{\tau} \\
& =(1 / t) \sum_{h \in \Lambda} \int_{\partial I_{h}} \frac{1}{\left|\widetilde{w}^{\prime}\right|} \frac{d \mathscr{H}^{0}}{\tau} \\
& \geq(2 / t) \sum_{h \in \Lambda} \operatorname{coth}\left((1 / 2) \mu\left(I_{h}\right)\right) \\
& \geq(2 / t) \operatorname{coth}\left((1 / 2) \sum_{h \in \Lambda} \mu\left(I_{h}\right)\right) \\
& =(2 / t) \operatorname{coth}((1 / 2) \mu(\{w>t\}))) \\
& =(2 / t) \operatorname{coth}\left((1 / 2) \mu_{w}(t)\right) .
\end{aligned}
$$

The statement now follows from Lemma 9.1.

(ii) Suppose that $\rho \not \equiv 0$ on $[a, b)$. Put $\alpha:=\min \{\rho>0\} \in[a, b)$. Now that $\{w>$ $t\} \uparrow(a, b)$ as $t \downarrow 1$ as $w>1$ on $(a, b)$. Choose $t_{0} \in\left(1,\|w\|_{\infty}\right)$ such that $\{w>$ $\left.t_{0}\right\} \cap(\alpha, b) \neq \emptyset$. Then for each $t \in\left(1, t_{0}\right)$ there exists $h \in \Lambda$ such that $\rho \neq \equiv 0$ on $I_{h}$. The statement then follows by Lemma 9.11 .

Lemma 9.14 Let $\emptyset \neq S \subset \mathbb{R}$ be bounded and suppose $S$ has the property that for each $s \in S$ there exists $\delta>0$ such that $[s, s+\delta) \subset S$. Then $S$ is $\mathscr{L}^{1}$-measurable and $|S|>0$. 
Proof For each $s \in S$ put $t_{s}:=\inf \{t>s: t \notin S\}$. Then $s<t_{s}<+\infty,\left[s, t_{s}\right) \subset S$ and $t_{s} \notin S$. Define

$$
\mathscr{C}:=\left\{[s, t]: s \in S \text { and } t \in\left(s, t_{s}\right)\right\}
$$

Then $\mathscr{C}$ is a Vitali cover of $S$ (see [6] Chapter 16 for example). By Vitali's Covering Theorem (cf. [6] Theorem 16.27) there exists an at most countably infinite subset $\Lambda \subset \mathscr{C}$ consisting of pairwise disjoint intervals such that

$$
\left|S \backslash \bigcup_{I \in \Lambda} I\right|=0 .
$$

Note that $I \subset S$ for each $I \in \Lambda$. Consequently, $S=\bigcup_{I \in \Lambda} I \cup N$ where $N$ is an $\mathscr{L}^{1}$-null set and hence $S$ is $\mathscr{L}^{1}$-measurable. The positivity assertion is clear.

Theorem 9.15 Let $0<a<b<+\infty$ and $\rho \geq 0$ be a non-decreasing bounded function on $[a, b]$. Let $(w, \lambda)$ solve (9.6). Assume that $w>1$ on $(a, b)$. Put $T:=$ $\min \left\{\left\|w_{0}\right\|_{\infty},\|w\|_{\infty}\right\}>1$. Then

(i) $\mu_{w}(t) \leq \mu_{w_{0}}(t)$ for each $t \in[1, T)$;

(ii) $\|w\|_{\infty} \leq\left\|w_{0}\right\|_{\infty}$;

(iii) if $\rho \not \equiv 0$ on $[a, b)$ then there exists $t_{0} \in\left(1,\|w\|_{\infty}\right)$ such that $\mu_{w}(t)<\mu_{w_{0}}(t)$ for each $t \in\left(1, t_{0}\right)$.

Proof (i) We adapt the proof of [16] Theorem I.6.1. The assumption entails that $\mu_{w}(1)=\mu_{w_{0}}(1)=\mu((a, b))$. Suppose for a contradiction that $\mu_{w}(t)>\mu_{w_{0}}(t)$ for some $t \in(1, T)$.

For $\varepsilon>0$ consider the initial value problem

$$
z^{\prime}=\omega(t, z)+\varepsilon \text { and } z(1)=\mu((a, b))+\varepsilon
$$

on $(0, T)$. Choose $v \in(0,1)$ and $\tau \in(t, T)$. By [16] Lemma I.3.1 there exists $\varepsilon_{0}>0$ such that for each $0 \leq \varepsilon<\varepsilon_{0}$ (9.11) has a continuously differentiable solution $z_{\varepsilon}$ defined on $[v, \tau]$ and this solution is unique by [16] Theorem I.3.1. Moreover, the sequence $\left(z_{\varepsilon}\right)_{0<\varepsilon<\varepsilon_{0}}$ converges uniformly to $z_{0}$ on $[v, \tau]$.

Given $0<\varepsilon<\eta<\varepsilon_{0}$ it holds that $z_{0} \leq z_{\varepsilon} \leq z_{\eta}$ on [1, $\tau$ ] by [16] Theorem I.6.1. Note for example that $z_{0}^{\prime} \leq \omega\left(\cdot, z_{0}\right)+\varepsilon$ on $(1, \tau)$. In fact, $\left(z_{\varepsilon}\right)_{0<\varepsilon<\varepsilon_{0}}$ decreases strictly to $z_{0}$ on $(1, \tau)$. For if, say, $z_{0}(s)=z_{\varepsilon}(s)$ for some $s \in(1, \tau)$ then $z_{\varepsilon}^{\prime}(s)=$ $\omega\left(s, z_{\varepsilon}(s)\right)+\varepsilon>\omega\left(s, z_{0}(s)\right)=z_{0}^{\prime}(s)$ by (9.11); while on the other hand $z_{\varepsilon}^{\prime}(s) \leq z_{0}^{\prime}(s)$ by considering the left-derivative at $s$ and using the fact that $z_{\varepsilon} \geq z_{0}$ on $[1, \tau]$. This contradicts the strict inequality.

Choose $\varepsilon_{1} \in\left(0, \varepsilon_{0}\right)$ such that $z_{\varepsilon}(t)<\mu_{w}(t)$ for each $0<\varepsilon<\varepsilon_{1}$. Now $\mu_{w}$ is right-continuous and strictly decreasing as $\mu_{w}(t)-\mu_{w}(s)=-\mu(\{s<w \leq t\})<0$ for $1 \leq s<t<\|w\|_{\infty}$ by continuity of $w$. So the set $\left\{z_{\varepsilon}<\mu_{w}\right\} \cap(1, t)$ is open and non-empty in $(0,+\infty)$ for each $\varepsilon \in\left(0, \varepsilon_{1}\right)$. Thus there exists a unique $s_{\varepsilon} \in[1, t)$ such that 


$$
\mu_{w}>z_{\varepsilon} \text { on }\left(s_{\varepsilon}, t\right] \text { and } \mu_{w}\left(s_{\varepsilon}\right)=z_{\varepsilon}\left(s_{\varepsilon}\right)
$$

for each $\varepsilon \in\left(0, \varepsilon_{1}\right)$. As $z_{\varepsilon}(1)>\mu((a, b))$ it holds that each $s_{\varepsilon}>1$. Note that $1<s_{\varepsilon}<s_{\eta}$ whenever $0<\varepsilon<\eta$ as $\left(z_{\varepsilon}\right)_{0<\varepsilon<\varepsilon_{0}}$ decreases strictly to $z_{0}$ as $\varepsilon \downarrow 0$.

Define

$$
S:=\left\{s_{\varepsilon}: 0<\varepsilon<\varepsilon_{1}\right\} \subset(1, t)
$$

We claim that for each $s \in S$ there exists $\delta>0$ such that $[s, s+\delta) \subset S$. This entails that $S$ is $\mathscr{L}^{1}$-measurable with positive $\mathscr{L}^{1}$-measure by Lemma 9.14.

Suppose $s=s_{\varepsilon} \in S$ for some $\varepsilon \in\left(0, \varepsilon_{1}\right)$ and put $z:=z_{\varepsilon}(s)=\mu_{w}(s)$. Put $k:=\operatorname{cosech}^{2}\left(z_{0}(t) / 2\right)$. For $0 \leq \zeta<\eta<\varepsilon_{1}$ define

$$
\Omega_{\zeta, \eta}:=\left\{(u, y) \in \mathbb{R}^{2}: u \in(0, t) \text { and } z_{\zeta}(u)<y<z_{\eta}(u)\right\}
$$

and note that this is an open set in $\mathbb{R}^{2}$. We remark that for each $(u, y) \in \Omega_{\zeta, \eta}$ there exists a unique $v \in(\zeta, \eta)$ such that $y=z_{v}(u)$. Given $r>0$ with $s+r<t$ set

$$
Q=Q_{r}:=\left\{(u, y) \in \mathbb{R}^{2}: s \leq u<s+r \text { and }|y-z|<\left\|z_{\varepsilon}-z\right\|_{C([s, s+r])}\right\} .
$$

Choose $r \in(0, t-s)$ and $\varepsilon_{2} \in\left(\varepsilon, \varepsilon_{1}\right)$ such that

(a) $Q_{r} \subset \Omega_{0, \varepsilon_{1}}$;

(b) $\left\|z_{\varepsilon}-z\right\|_{C([s, s+r])}<s \varepsilon /(2 k)$;

(c) $\sup _{\eta \in\left(\varepsilon, \varepsilon_{2}\right)}\left\|z_{\eta}-z\right\|_{C([s, s+r])} \leq\left\|z_{\varepsilon}-z\right\|_{C([s, s+r])}$;

(d) $z_{\eta}<\mu_{w}$ on $[s+r, t]$ for each $\eta \in\left(\varepsilon, \varepsilon_{2}\right)$.

We can find $\delta \in(0, r)$ such that $z_{\varepsilon}<\mu_{w}<z_{\varepsilon_{2}}$ on $(s, s+\delta)$ as $z_{\varepsilon_{2}}(s)>z$; in other words, the graph of $\mu_{w}$ restricted to $(s, s+\delta)$ is contained in $\Omega_{\varepsilon, \varepsilon_{2}}$.

Let $u \in(s, s+\delta)$. Then $\mu_{w}(u)=z_{\eta}(u)$ for some $\eta \in\left(\varepsilon, \varepsilon_{2}\right)$ as above. We claim that $u=s_{\eta}$ so that $u \in S$. This implies in turn that $[s, s+\delta) \subset S$. Suppose for a contradiction that $z_{\eta} \nless \mu_{w}$ on $(u, t]$. Then there exists $v \in(u, t]$ such that $\mu_{w}(v)=z_{\eta}(v)$. In view of condition (d), $v \in(u, s+r)$. By [1] Theorem 3.28 and Theorem 9.13,

$$
\begin{aligned}
\mu_{w}(v)-\mu_{w}(u) & =D \mu_{w}((u, v])=D \mu_{w}^{a}((u, v])+D \mu_{w}^{s}((u, v]) \\
& \leq D \mu_{w}^{a}((u, v])=\int_{u}^{v} \mu_{w}^{\prime} d \tau \leq \int_{u}^{v} \omega\left(\cdot, \mu_{w}\right) d \tau
\end{aligned}
$$

On the other hand,

$$
z_{\eta}(v)-z_{\eta}(u)=\int_{u}^{v} z_{\eta}^{\prime} d \tau=\int_{u}^{v} \omega\left(\cdot, z_{\eta}\right) d \tau+\eta(v-u)
$$


We derive that

$$
\begin{aligned}
\varepsilon(v-u) & \leq \eta(v-u) \leq \int_{u}^{v}\left\{\omega\left(\cdot, \mu_{w}\right)-\omega\left(\cdot, z_{\eta}\right)\right\} d \tau \\
& \leq k \int_{u}^{v}\left|\mu_{w}-z_{\eta}\right| d \mu
\end{aligned}
$$

using the estimate (9.7). Thus

$$
\begin{aligned}
\varepsilon & \leq k \frac{1}{v-u} \int_{u}^{v}\left|\mu_{w}-z_{\eta}\right| d \mu \\
& \leq(k / s)\left\|\mu_{w}-z_{\eta}\right\|_{C([u, v])} \\
& \leq(k / s)\left\{\left\|\mu_{w}-z\right\|_{C([s, s+r])}+\left\|z_{\eta}-z\right\|_{C([s, s+r])}\right\} \\
& \leq(2 k / s)\left\|z_{\varepsilon}-z\right\|_{C([s, s+r])}<\varepsilon
\end{aligned}
$$

by (b) and (c) giving rise to the desired contradiction.

By Theorem 9.13, $\mu_{w}^{\prime} \leq \omega\left(\cdot, \mu_{w}\right)$ for $\mathscr{L}^{1}$-a.e. $t \in S$. Choose $s \in S$ such that $\mu_{w}$ is differentiable at $s$ and the latter inequality holds at $s$. Let $\varepsilon \in\left(0, \varepsilon_{1}\right)$ such that $s=s_{\varepsilon}$. For any $u \in(s, t)$,

$$
\mu_{w}(u)-\mu_{w}(s)>z_{\varepsilon}(u)-z_{\varepsilon}(s)
$$

We deduce that $\mu_{w}^{\prime}(s) \geq z_{\varepsilon}^{\prime}(s)$. But then

$$
\mu_{w}^{\prime}(s) \geq z_{\varepsilon}^{\prime}(s)=\omega\left(s, z_{\epsilon}(s)\right)+\varepsilon>\omega\left(s, \mu_{w}(s)\right) .
$$

This strict inequality holds on a set of full measure in $S$. This contradicts Theorem 9.13 .

(ii) Use the fact that $\|w\|_{\infty}=\sup \left\{t>0: \mu_{w}(t)>0\right\}$.

(iii) Assume that $\rho \not \equiv 0$ on $[a, b)$. Let $t_{0} \in\left(1,\|w\|_{\infty}\right)$ be as in Lemma 9.13. Then for $t \in\left(1, t_{0}\right)$,

$$
\begin{aligned}
\mu_{w}(t)-\mu_{w}(1)= & D \mu_{w}((1, t])=D \mu_{w}^{a}((1, t]) \\
& +D \mu_{w}^{s}((1, t]) \leq D \mu_{w}^{a}((1, t]) \\
= & \int_{(1, t]} \mu_{w}^{\prime} d s<\int_{(1, t]} \omega\left(s, \mu_{w}\right) d s \\
\leq & \int_{(1, t]} \omega\left(s, \mu_{w_{0}}\right) d s=\mu_{w_{0}}(t)-\mu_{w_{0}}(1)
\end{aligned}
$$

by Theorem 9.13, Lemma 9.9 and the inequality in $(i)$. 
Corollary 9.16 Let $0<a<b<+\infty$ and $\rho \geq 0$ be a non-decreasing bounded function on $[a, b]$. Suppose that $(w, \lambda)$ solves (9.6). Assume that $w>1$ on $(a, b)$. Let $0 \leq \varphi \in C^{1}((1,+\infty))$ be strictly decreasing with $\int_{a}^{b} \varphi\left(w_{0}\right) d \mu<+\infty$. Then

(i) $\int_{a}^{b} \varphi(w) d \mu \geq \int_{a}^{b} \varphi\left(w_{0}\right) d \mu$;

(ii) equality holds in (i) if and only if $\rho \equiv 0$ on $[a, b)$.

In particular,

(iii) $\int_{a}^{b} \frac{1}{\sqrt{w^{2}-1}} d \mu \geq \pi$ with equality if and only if $\rho \equiv 0$ on $[a, b)$.

Proof (i) Let $\varphi \geq 0$ be a decreasing function on $(1,+\infty)$ which is piecewise $C^{1}$. Suppose that $\varphi(1+)<+\infty$. By Tonelli's Theorem,

$$
\begin{aligned}
\int_{[1,+\infty)} \varphi^{\prime} \mu_{w} d s & =\int_{[1,+\infty)} \varphi^{\prime}\left\{\int_{(a, b)} \chi_{\{w>s\}} d \mu\right\} d s \\
& =\int_{(a, b)}\left\{\int_{[1,+\infty)} \varphi^{\prime} \chi_{\{w>s\}} d s\right\} d \mu \\
& =\int_{(a, b)}\{\varphi(w)-\varphi(1)\} d \mu \\
& =\int_{(a, b)} \varphi(w) d \mu-\varphi(1) \mu((a, b))
\end{aligned}
$$

and a similar identity holds for $\mu_{w_{0}}$. By Theorem 9.15, $\int_{a}^{b} \varphi(w) d \mu \geq \int_{a}^{b} \varphi\left(w_{0}\right) d \mu$. Now suppose that $0 \leq \varphi \in C^{1}((1,+\infty))$ is strictly decreasing with $\int_{a}^{b} \varphi\left(w_{0}\right) d \mu<$ $+\infty$. The inequality holds for the truncated function $\varphi \wedge n$ for each $n \in \mathbb{N}$. An application of the monotone convergence theorem establishes the result for $\varphi$.

(ii) Suppose that equality holds in $(i)$. For $c \in(1,+\infty)$ put $\varphi_{1}:=\varphi \vee \varphi(c)-\varphi(c)$ and $\varphi_{2}:=\varphi \wedge \varphi(c)$. By $(i)$ we deduce

$$
\int_{a}^{b} \varphi_{2}(w) d \mu=\int_{a}^{b} \varphi_{2}\left(w_{0}\right) d \mu
$$

and hence by the above that

$$
\int_{[c,+\infty)} \varphi^{\prime}\left\{\mu_{w}-\mu_{w_{0}}\right\} d s=0 .
$$

This means that $\mu_{w}=\mu_{w_{0}}$ on $(c,+\infty)$ and hence on $(1,+\infty)$. By Theorem 9.15 we conclude that $\rho \equiv 0$ on $[a, b)$. (iii) flows from $(i)$ and (ii) noting that the function $\varphi:(1,+\infty) \rightarrow \mathbb{R} ; t \mapsto 1 / \sqrt{t^{2}-1}$ satisfies the integral condition by Lemma 9.9.

The case $a=0$. Let $0<b<+\infty$ and $\rho \geq 0$ be a non-decreasing bounded function on $[0, b]$. We study solutions to the first-order linear ordinary differential equation

$$
u^{\prime}+(1 / x+\rho) u+\lambda=0 \text { a.e. on }(0, b) \text { with } u(0)=0 \text { and } u(b)=1
$$


where $u \in C^{0,1}([0, b])$ and $\lambda \in \mathbb{R}$. If $\rho \equiv 0$ on $[0, b]$ then we write $u_{0}$ instead of $u$.

Lemma 9.17 Let $0<b<+\infty$ and $\rho \geq 0$ be a non-decreasing bounded function on $[0, b]$. Then

(i) there exists a solution $(u, \lambda)$ of (9.12) with $u \in C^{0,1}([0, b])$ and $\lambda \in \mathbb{R}$;

(ii) $\lambda$ is given by $\lambda=-g(b) / G(b)$ where $G:=\int_{0}^{\cdot} g d s$;

(iii) the pair $(u, \lambda)$ in (i) is unique;

(iv) $u>0$ on $(0, b]$.

Proof (i) The function $u:[a, b] \rightarrow \mathbb{R}$ given by

$$
u=\frac{g(b)}{G(b)} \frac{G}{g}
$$

on $[0, b]$ solves $(9.12)$ with $\lambda$ as in (ii). (iii) Suppose that $\left(u_{1}, \lambda_{1}\right)$ resp. $\left(u_{2}, \lambda_{2}\right)$ solve (9.12). By linearity $u:=u_{1}-u_{2}$ solves

$$
u^{\prime}+(1 / x+\rho) u+\lambda=0 \text { a.e. on }(0, b) \text { with } u(0)=u(b)=0
$$

where $\lambda=\lambda_{1}-\lambda_{2}$. An integration gives that $u=(-\lambda G+c) / g$ for some constant $c \in \mathbb{R}$ and the boundary conditions entail that $\lambda=c=0$. (iv) follows from the formula (9.13) and unicity.

Lemma 9.18 Suppose $-\infty<a<b<+\infty$ and that $\phi:[a, b] \rightarrow \mathbb{R}$ is convex. Suppose that there exists $\xi \in(a, b)$ such that

$$
\phi(\xi)=\frac{b-\xi}{b-a} \phi(a)+\frac{\xi-a}{b-a} \phi(b) .
$$

Then

$$
\phi(c)=\frac{b-c}{b-a} \phi(a)+\frac{c-a}{b-a} \phi(b)
$$

for each $c \in[a, b]$.

Proof Let $c \in(\xi, b)$. By monotonicity of chords,

$$
\frac{\phi(\xi)-\phi(a)}{\xi-a} \leq \frac{\phi(c)-\phi(\xi)}{c-\xi}
$$

so

$$
\begin{aligned}
\phi(c) & \geq \frac{c-a}{\xi-a} \phi(\xi)-\frac{c-\xi}{\xi-a} \phi(a) \\
& =\frac{c-a}{\xi-a}\left\{\frac{b-\xi}{b-a} \phi(a)+\frac{\xi-a}{b-a} \phi(b)\right\}-\frac{c-\xi}{\xi-a} \phi(a)
\end{aligned}
$$




$$
=\frac{b-c}{b-a} \phi(a)+\frac{c-a}{b-a} \phi(b)
$$

and equality follows. The case $c \in(a, \xi)$ is similar.

Lemma 9.19 Let $0<b<+\infty$ and $\rho \geq 0$ be a non-decreasing bounded function on $[0, b]$. Let $(u, \lambda)$ satisfy (9.12). Then

(i) $u \geq u_{0}$ on $[0, b]$;

(ii) if $\rho \not \equiv 0$ on $[0, b)$ then $u>u_{0}$ on $(0, b)$.

Proof (i) The mapping $G:[0, b] \rightarrow[0, G(b)]$ is a bijection with inverse $G^{-1}$. Define $\eta:[0, G(b)] \rightarrow \mathbb{R}$ via $\eta:=(\operatorname{tg}) \circ G^{-1}$. Then

$$
\eta^{\prime}=\frac{(\operatorname{tg})^{\prime}}{g} \circ G^{-1}=(2+t \rho) \circ G^{-1}
$$

a.e. on $(0, G(b))$ so $\eta^{\prime}$ is non-decreasing there. This means that $\eta$ is convex on $[0, G(b)]$. In particular, $\eta(s) \leq[\eta(G(b)) / G(b)] s$ for each $s \in[0, G(b)]$. For $t \in[0, b]$ put $s:=G(t)$ to obtain $\operatorname{tg}(t) \leq(b g(b) / G(b)) G(t)$. A rearrangement gives $u \geq u_{0}$ on $[0, b]$ noting that $u_{0}:[0, b] \rightarrow \mathbb{R} ; t \mapsto t / b$. (ii) Assume $\rho \neq \equiv 0$ on $[0, b)$. Suppose that $u(c)=u_{0}(c)$ for some $c \in(0, b)$. Then $\eta(G(c))=[\eta(G(b)) / G(b)] G(c)$. By Lemma $9.18, \eta^{\prime}=0$ on $(0, G(b))$. This implies that $\rho \equiv 0$ on $[0, b)$.

Lemma 9.20 Let $0<b<+\infty$. Then $\int_{0}^{b} \frac{u_{0}}{\sqrt{1-u_{0}^{2}}} d \mu=\pi / 2$.

Proof The integral is elementary as $u_{0}(t)=t / b$ for $t \in[0, b]$.

\section{Proof of main results}

Lemma 10.1 Let $x \in H$ and $v$ be a unit vector in $\mathbb{R}^{2}$ such that the pair $\{x, v\}$ forms a positively oriented orthogonal basis for $\mathbb{R}^{2}$. Put $b:=(\tau, 0)$ where $|x|=\tau$ and $\gamma:=\theta(x) \in(0, \pi)$. Let $\alpha \in(0, \pi / 2)$ such that

$$
\frac{\langle v, x-b\rangle}{|x-b|}=\cos \alpha .
$$

Then

(i) $C(x, v, \alpha) \cap H \cap \bar{C}\left(0, e_{1}, \gamma\right)=\emptyset$;

(ii) for any $y \in C(x, v, \alpha) \cap H \backslash \bar{B}(0, \tau)$ the line segment $[b, y]$ intersects $\mathbb{S}_{\tau}^{1}$ outside the closed cone $\bar{C}\left(0, e_{1}, \gamma\right)$.

We point out that $C\left(0, e_{1}, \gamma\right)$ is the open cone with vertex 0 and axis $e_{1}$ which contains the point $x$ on its boundary. We note that $\cos \alpha \in(0,1)$ because 


$$
\begin{aligned}
\langle v, x-b\rangle & =-\langle v, b\rangle=-\langle(1 / \tau) O x, b\rangle \\
& =-\left\langle O p, e_{1}\right\rangle=\left\langle x, O^{\star} e_{1}\right\rangle=\left\langle x, e_{2}\right\rangle>0
\end{aligned}
$$

and if $|x-b|=\langle v, x-b\rangle$ then $b=x-\lambda v$ for some $\lambda \in \mathbb{R}$ and hence $x_{1}=\left\langle e_{1}, x\right\rangle=\tau$ and $x_{2}=0$.

Proof (i) For $\omega \in \mathbb{S}^{1}$ define the open half-space

$$
H_{\omega}:=\left\{y \in \mathbb{R}^{2}:\langle y, \omega\rangle>0\right\} .
$$

We claim that $C(x, v, \alpha) \subset H_{v}$. For given $y \in C(x, v, \alpha)$,

$$
\langle y, v\rangle=\langle y-x, v\rangle>|y-x| \cos \alpha>0 .
$$

On the other hand, it holds that $\bar{C}\left(0, e_{1}, \gamma\right) \cap H \subset \bar{H}_{-v}$. This establishes $(i)$.

(ii) By some trigonometry $\gamma=2 \alpha$. Suppose that $\omega$ is a unit vector in $C\left(b,-e_{1}, \pi / 2-\right.$ $\alpha)$. Then $\lambda:=\left\langle\omega, e_{1}\right\rangle<\cos \alpha$ since upon rewriting the membership condition for $C\left(b,-e_{1}, \pi / 2-\alpha\right)$ we obtain the quadratic inequality

$$
\lambda^{2}-2 \cos ^{2} \alpha \lambda+\cos \gamma>0
$$

For $\omega$ a unit vector in $\bar{C}\left(0, e_{1}, \gamma\right)$ the opposite inequality $\left\langle\omega, e_{1}\right\rangle \geq \cos \alpha$ holds. This shows that

$$
C\left(b,-e_{1}, \pi / 2-\alpha\right) \cap \bar{C}\left(0, e_{1}, \gamma\right) \cap \mathbb{S}_{\tau}^{1}=\emptyset .
$$

The set $C(x, v, \alpha) \cap H$ is contained in the open convex cone $C\left(b,-e_{1}, \pi / 2-\alpha\right)$. Suppose $y \in C(x, v, \alpha) \cap H \backslash \bar{B}(0, \tau)$. Then the line segment $[b, y]$ is contained in $C\left(b,-e_{1}, \pi / 2-\alpha\right) \cup\{b\}$. Now the set $C\left(b,-e_{1}, \pi / 2-\alpha\right) \cap \mathbb{S}_{\tau}^{1}$ disconnects $C\left(b,-e_{1}, \pi / 2-\alpha\right) \cup\{b\}$. This entails that $(b, y] \cap C\left(b,-e_{1}, \pi / 2-\alpha\right) \cap \mathbb{S}_{\tau}^{1} \neq \emptyset$. The foregoing paragraph entails that $(b, y] \cap \bar{C}\left(0, e_{1}, \gamma\right) \cap \mathbb{S}_{\tau}^{1}=\emptyset$. This establishes the result.

Lemma 10.2 Let $E$ be an open set in $\mathbb{R}^{2}$ such that $M:=\partial E$ is a $C^{1,1}$ hypersurface in $\mathbb{R}^{2}$. Assume that $E \backslash\{0\}=E^{s c}$. Suppose

(i) $x \in(M \backslash\{0\}) \cap H$;

(ii) $\sin (\sigma(x))=-1$.

Then $E$ is not convex.

Proof Let $\gamma_{1}: I \rightarrow M$ be a $C^{1,1}$ parametrisation of $M$ in a neighbourhood of $x$ with $\gamma_{1}(0)=x$ as above. As $\sin (\sigma(x))=-1, n(x)$ and hence $n_{1}(0)$ point in the direction of $x$. Put $v:=-t_{1}(0)=-t(x)$. We may write

$$
\gamma_{1}(s)=\gamma_{1}(0)+s t_{1}(0)+R_{1}(s)=x-s v+R_{1}(s)
$$


for $s \in I$ where $R_{1}(s)=s \int_{0}^{1} \dot{\gamma}_{1}(t s)-\dot{\gamma}_{1}(0) d t$ and we can find a finite positive constant $K$ such that $\left|R_{1}(s)\right| \leq K s^{2}$ on a symmetric open interval $I_{0}$ about 0 with $I_{0} \subset \subset I$. Then

$$
\begin{aligned}
\frac{\left\langle\gamma_{1}(s)-x, v\right\rangle}{\left|\gamma_{1}(s)-x\right|} & =\frac{\left\langle-s v+R_{1}, v\right\rangle}{\left|-s v+R_{1}\right|} \\
& =\frac{1-\left\langle\left(R_{1} / s\right), v\right\rangle}{\left|v-R_{1} / s\right|} \rightarrow 1
\end{aligned}
$$

as $s \uparrow 0$. Let $\alpha$ be as in Lemma 10.1 with $x$ and $v$ as just mentioned. The above estimate entails that $\gamma_{1}(s) \in C(x, v, \alpha)$ for small $s<0$. By (2.9) and Lemma 5.4 the function $r_{1}$ is non-increasing on $I$. In particular, $r_{1}(s) \geq r_{1}(0)=|x|=$ : $\tau$ for $I \ni s<0$ and $\gamma_{1}(s) \notin B(0, \tau)$.

Choose $\delta_{1}>0$ such that $\gamma_{1}(s) \in C(x, v, \alpha) \cap H$ for each $s \in\left[-\delta_{1}, 0\right)$. Put $\beta:=\inf \left\{s \in\left[-\delta_{1}, 0\right]: r_{1}(s)=\tau\right\}$. Suppose first that $\beta \in\left[-\delta_{1}, 0\right)$. Then $E$ is not convex (see Lemma 5.2). Now suppose that $\beta=0$. Let $\gamma$ be as in Lemma 10.1. Then the open circular arc $\mathbb{S}_{\tau}^{1} \backslash \bar{C}\left(0, e_{1}, \gamma\right)$ does not intersect $\bar{E}$ : for otherwise, $M$ intersects $\mathbb{S}_{\tau}^{1} \backslash \bar{C}\left(0, e_{1}, \gamma\right)$ and $\beta<0$ bearing in mind Lemma 5.2. Choose $s \in\left[-\delta_{1}, 0\right)$. Then the points $b$ and $\gamma_{1}(s)$ lie in $\bar{E}$. But by Lemma 10.1 the line segment $\left[b, \gamma_{1}(s)\right]$ intersects $\mathbb{S}_{\tau}^{1}$ in $\mathbb{S}_{\tau}^{1} \backslash \bar{C}\left(0, e_{1}, \gamma\right)$. Let $c \in\left[b, \gamma_{1}(s)\right] \cap \mathbb{S}_{\tau}^{1}$. Then $c \notin \bar{E}$. This shows that $\bar{E}$ is not convex. But if $E$ is convex then $\bar{E}$ is convex. Therefore $E$ is not convex.

Theorem 10.3 Let $f$ be as in (1.3) where $h:[0,+\infty) \rightarrow \mathbb{R}$ is a non-decreasing convex function. Given $v>0$ let $E$ be a bounded minimiser of (1.2). Assume that $E$ is open, $M:=\partial E$ is a $C^{1,1}$ hypersurface in $\mathbb{R}^{2}$ and $E \backslash\{0\}=E^{s c}$. Put

$$
R:=\inf \{\rho>0\} \in[0,+\infty)
$$

Then $\Omega \cap(R,+\infty)=\emptyset$ with $\Omega$ as in (5.2).

Proof Suppose that $\Omega \cap(R,+\infty) \neq \emptyset$. As $\Omega$ is open in $(0,+\infty)$ by Lemma 5.6 we may write $\Omega$ as a countable union of disjoint open intervals in $(0,+\infty)$. By a suitable choice of one of these intervals we may assume that $\Omega=(a, b)$ for some $0 \leq a<b<+\infty$ and that $\Omega \cap(R,+\infty) \neq \emptyset$. Let us assume for the time being that $a>0$. Note that $[a, b] \subset \pi(M)$ and $\cos \sigma$ vanishes on $M_{a} \cup M_{b}$.

Let $u: \Omega \rightarrow[-1,1]$ be as in (6.6). Then $u$ has a continuous extension to $[a, b]$ and $u= \pm 1$ at $\tau=a, b$. This may be seen as follows. For $\tau \in(a, b)$ the set $M_{\tau} \cap \bar{H}$ consists of a singleton by Lemma 5.4. The limit $x:=\lim _{\tau \downarrow a} M_{\tau} \cap \bar{H} \in \mathbb{S}_{a}^{1} \cap \bar{H}$ exists as $M$ is $C^{1}$. There exists a $C^{1,1}$ parametrisation $\gamma_{1}: I \rightarrow M$ with $\gamma_{1}(0)=x$ as above. By (2.9) and Lemma 5.4, $r_{1}$ is decreasing on $I$. So $r_{1}>a$ on $I \cap\{s<0\}$ for otherwise the $C^{1}$ property fails at $x$. It follows that $\gamma_{1}=\gamma \circ r_{1}$ and $\sigma_{1}=\sigma \circ \gamma \circ r_{1}$ on $I \cap\{s<0\}$. Thus $\sin (\sigma \circ \gamma) \circ r_{1}=\sin \sigma_{1}$ on $I \cap\{s<0\}$. Now the function $\sin \sigma_{1}$ is continuous on $I$. So $u \rightarrow \sin \sigma_{1}(0) \in\{ \pm 1\}$ as $\tau \downarrow a$. Put $\eta_{1}:=u(a)$ and $\eta_{2}:=u(b)$.

Let us consider the case $\eta=\left(\eta_{1}, \eta_{2}\right)=(1,1)$. According to Theorem 6.5 the generalised (mean) curvature is constant $\mathscr{H}^{1}$-a.e. on $M$ with value $-\lambda$, say. Note that $u<1$ on $(a, b)$ for otherwise $\cos (\sigma \circ \gamma)$ vanishes at some point in $(a, b)$ bearing in 
mind Lemma 5.4. By Theorem 6.6 the pair $(u, \lambda)$ satisfies $(9.4)$ with $\eta=(1,1)$. By Lemma 9.2, $u>0$ on $[a, b]$. Put $w:=1 / u$. Then $(w,-\lambda)$ satisfies (9.6) and $w>1$ on $(a, b)$. By Lemma 6.7,

$$
\begin{aligned}
\theta_{2}(b)-\theta_{2}(a) & =\int_{a}^{b} \theta_{2}^{\prime} d \tau=-\int_{a}^{b} \frac{u}{\sqrt{1-u^{2}}} \frac{d \tau}{\tau} \\
& =-\int_{a}^{b} \frac{1}{\sqrt{w^{2}-1}} \frac{d \tau}{\tau} .
\end{aligned}
$$

By Corollary 9.16, $\left|\theta_{2}(b)-\theta_{2}(a)\right|>\pi$. But this contradicts the definition of $\theta_{2}$ in (6.4) as $\theta_{2}$ takes values in $(0, \pi)$ on $(a, b)$. If $\eta=(-1,-1)$ then $\lambda>0$ by Lemma 9.2; this contradicts Lemma 7.2.

Now let us consider the case $\eta=(-1,1)$. Using the same formula as above, $\theta_{2}(b)-\theta_{2}(a)<0$ by Corollary 9.7. This means that $\theta_{2}(a) \in(0, \pi]$. As before the limit $x:=\lim _{\tau \downarrow a} M_{\tau} \cap \bar{H} \in \mathbb{S}_{a}^{1} \cap \bar{H}$ exists as $M$ is $C^{1}$. Using a local parametrisation it can be seen that $\theta_{2}(a)=\theta(x)$ and $\sin (\sigma(x))=-1$. If $\theta_{2}(a) \in(0, \pi)$ then $E$ is not convex by Lemma 10.2. This contradicts Theorem 7.3. Note that we may assume that $\theta_{2}(a) \in(0, \pi)$. For otherwise, $\left\langle\gamma, e_{2}\right\rangle<0$ for $\tau>a$ near $a$, contradicting the definition of $\gamma(6.5)$. If $\eta=(1,-1)$ then $\lambda>0$ by Lemma 9.2 and this contradicts Lemma 7.2 as before.

Suppose finally that $a=0$. By Lemma 5.5, $u(0)=0$ and $u(b)= \pm 1$. Suppose $u(b)=1$. Again employing the formula above, $\theta_{2}(b)-\theta_{2}(0)<-\pi / 2$ by Lemma 9.19, the fact that the function $\phi:(0,1) \rightarrow \mathbb{R} ; t \mapsto t / \sqrt{1-t^{2}}$ is strictly increasing and Lemma 9.20. This means that $\theta_{2}(0)>\pi / 2$. This contradicts the $C^{1}$ property at $0 \in M$. If $u(b)=-1$ then then $\lambda>0$ by Lemma 9.2 giving a contradiction.

Lemma 10.4 Let $f$ be as in (1.3) where $h:[0,+\infty) \rightarrow \mathbb{R}$ is a non-decreasing convex function. Let $v>0$.

(i) Let $E$ be a bounded minimiser of (1.2). Assume that $E$ is open, $M:=\partial E$ is a $C^{1,1}$ hypersurface in $\mathbb{R}^{2}$ and $E \backslash\{0\}=E^{s c}$. Thenfor any $r>0$ with $r \geq R, M \backslash \bar{B}(0, r)$ consists of a finite union of disjoint centred circles.

(ii) There exists a minimiser $E$ of (1.2) such that $\partial E$ consists of a finite union of disjoint centred circles.

Proof (i) First observe that

$$
\emptyset \neq \pi(M)=[\pi(M) \cap[0, r]] \cup[\pi(M) \cap(r,+\infty)] \backslash \Omega
$$

by Lemma 10.3. We assume that the latter member is non-empty. By definition of $\Omega, \cos \sigma=0$ on $M \cap A((r,+\infty))$. Let $\tau \in \pi(M) \cap(r,+\infty)$. We claim that $M_{\tau}=\mathbb{S}_{\tau}^{1}$. Suppose for a contradiction that $M_{\tau} \neq \mathbb{S}_{\tau}^{1}$. By Lemma 5.2, $M_{\tau}$ is the union of two closed spherical arcs in $\mathbb{S}_{\tau}^{1}$. Let $x$ be a point on the boundary of one of these spherical arcs relative to $\mathbb{S}_{\tau}^{1}$. There exists a $C^{1,1}$ parametrisation $\gamma_{1}: I \rightarrow M$ of $M$ in a neighbourhood of $x$ with $\gamma_{1}(0)=x$ as before. By shrinking $I$ if necessary we may assume that $\gamma_{1}(I) \subset A((r,+\infty))$ as $\tau>r$. By (2.9), $\dot{r}_{1}=0$ on $I$ as $\cos \sigma_{1}=0$ on $I$ 
because $\cos \sigma=0$ on $M \cap A((r,+\infty))$; that is, $r_{1}$ is constant on $I$. This means that $\gamma_{1}(I) \subset \mathbb{S}_{\tau}^{1}$. As the function $\sin \sigma_{1}$ is continuous on $I$ it takes the value \pm 1 there. By (2.10), $r_{1} \dot{\theta}_{1}=\sin \sigma_{1}= \pm 1$ on $I$. This means that $\theta_{1}$ is either strictly decreasing or strictly increasing on $I$. This entails that the point $x$ is not a boundary point of $M_{\tau}$ in $\mathbb{S}_{\tau}^{1}$ and this proves the claim.

It follows from these considerations that $M \backslash \bar{B}(0, r)$ consists of a finite union of disjoint centred circles. Note that $f \geq e^{h(0)}=: c>0$ on $\mathbb{R}^{2}$. As a result, $+\infty>$ $P_{f}(E) \geq c P(E)$ and in particular the relative perimeter $P\left(E, \mathbb{R}^{2} \backslash \bar{B}(0, r)\right)<+\infty$. This explains why $M \backslash \bar{B}(0, r)$ comprises only finitely many circles.

(ii) Let $E$ be a bounded minimiser of (1.2) such that $E$ is open, $M:=\partial E$ is a $C^{1,1}$ hypersurface in $\mathbb{R}^{2}$ and $E \backslash\{0\}=E^{s c}$ as in Theorem 4.5. Assume that $R>0$. By $(i)$, $M \backslash \bar{B}(0, R)$ consists of a finite union of disjoint centred circles. We claim that only one of the possibilities

$$
M_{R}=\emptyset, M_{R}=\mathbb{S}_{R}^{1}, M_{R}=\left\{R e_{1}\right\} \text { or } M_{R}=\left\{-R e_{1}\right\}
$$

holds. To prove this suppose that $M_{R} \neq \emptyset$ and $M_{R} \neq \mathbb{S}_{R}^{1}$. Bearing in mind Lemma 5.2 we may choose $x \in M_{R}$ such that $x$ lies on the boundary of $M_{R}$ relative to $\mathbb{S}_{R}^{1}$. Assume that $x \in H$. Let $\gamma_{1}: I \rightarrow M$ be a local parametrisation of $M$ with $\gamma_{1}(0)=x$ with the usual conventions. We first notice that $\cos (\sigma(x))=0$ for otherwise we obtain a contradiction to Theorem 10.3. As $r_{1}$ is decreasing on $I$ and $x$ is a relative boundary point it holds that $r_{1}<R$ on $I^{+}:=I \cap\{s>0\}$. As $M \backslash \overline{\Lambda_{1}}$ is open in $M$ we may suppose that $\gamma_{1}\left(I^{+}\right) \subset M \backslash \overline{\Lambda_{1}}$. According to Theorem 6.5 the curvature $k$ of $\gamma_{1}\left(I^{+}\right) \cap B(0, R)$ is a.e. constant as $\rho$ vanishes on $(0, R)$. Hence $\gamma_{1}\left(I^{+}\right) \cap B(0, R)$ consists of a line or circular arc. The fact that $\cos (\sigma(x))=0$ means that $\gamma_{1}\left(I^{+}\right) \cap B(0, R)$ cannot be a line. So $\gamma_{1}\left(I^{+}\right) \cap B(0, R)$ is an open arc of a circle $C$ containing $x$ in its closure with centre on the line-segment $[0, x]$ and radius $r \in(0, R)$. By considering a local parametrisation, it can be seen that $C \cap B(0, R) \subset M$. But this contradicts the fact that $E \backslash\{0\}=E^{s c}$. In summary, $M_{R} \subset\left\{ \pm R e_{1}\right\}$. Finally note that if $M_{R}=\left\{ \pm R e_{1}\right\}$ then $M_{R}=\mathbb{S}_{R}^{1}$ by Lemma 5.2. This establishes (10.3).

Suppose that $M_{R}=\emptyset$. As both sets $M$ and $\mathbb{S}_{R}^{1}$ are compact, $d\left(M, \mathbb{S}_{R}^{1}\right)>0$. Assume first that $\mathbb{S}_{R}^{1} \subset E$. Put $F:=B(0, R) \backslash E$ and suppose $F \neq \emptyset$. Then $F$ is a set of finite perimeter, $F \subset \subset B(0, R)$ and $P(F)=P(E, B(0, R))$. Let $B$ be a centred ball with $|B|=|F|$. By the classical isoperimetric inequality, $P(B) \leq P(F)$. Define $E_{1}:=\left(\mathbb{R}^{2} \backslash B\right) \cap(B(0, R) \cup E)$. Then $V_{f}\left(E_{1}\right)=V_{f}(E)$ and $P_{f}\left(E_{1}\right) \leq P_{f}(E)$. That is, $E_{1}$ is a minimiser of (1.2) such that $\partial E_{1}$ consists of a finite union of disjoint centred circles. Now suppose that $\mathbb{S}_{R}^{1} \subset \mathbb{R}^{2} \backslash \bar{E}$. In like fashion we may redefine $E$ via $E_{1}:=B \cup(E \backslash \bar{B}(0, R))$ with $B$ a centred ball in $B(0, R)$. The remaining cases in (10.3) can be dealt with in a similar way. The upshot of this argument is that there exists a m inimiser of (1.2) whose boundary $M$ consists of a finite union of disjoint centred circles in case $R>0$.

Now suppose that $R=0$. By $(i), M \backslash \bar{B}(0, r)$ consists of a finite union of disjoint centred circles for any $r \in(0,1)$. If these accumulate at 0 then $M$ fails to be $C^{1}$ at the origin. The assertion follows. 
Lemma 10.5 Suppose that the function $J:[0,+\infty) \rightarrow[0,+\infty)$ is continuous nondecreasing and $J(0)=0$. Let $N \in \mathbb{N} \cup\{+\infty\}$ and $\left\{t_{h}: h=0, \ldots, 2 N+1\right\} a$ sequence of points in $[0,+\infty)$ with

$$
t_{0}>t_{1}>\cdots>t_{2 h}>t_{2 h+1}>\cdots \geq 0
$$

Then

$$
+\infty \geq \sum_{h=0}^{2 N+1} J\left(t_{h}\right) \geq J\left(\sum_{h=0}^{2 N+1}(-1)^{h} t_{h}\right) .
$$

Proof We suppose that $N=+\infty$. The series $\sum_{h=0}^{\infty}(-1)^{h} t_{h}$ converges by the alternating series test. For each $n \in \mathbb{N}$,

$$
\sum_{h=0}^{2 n+1}(-1)^{h} t_{h} \leq t_{0}
$$

and the same inequality holds for the infinite sum. As in Step 2 in [5] Theorem 2.1,

$$
+\infty \geq \sum_{h=0}^{\infty} J\left(t_{h}\right) \geq J\left(t_{0}\right) \geq J\left(\sum_{h=0}^{\infty}(-1)^{h} t_{h}\right)
$$

as $J$ is non-decreasing.

Proof of Theorem 1.1 There exists a minimiser $E$ of (1.2) with the property that $\partial E$ consists of a finite union of disjoint centred circles according to Lemma 10.4. As such we may write

$$
E=\bigcup_{h=0}^{N} A\left(\left(a_{2 h+1}, a_{2 h}\right)\right)
$$

where $N \in \mathbb{N}$ and $+\infty>a_{0}>a_{1}>\cdots>a_{2 N}>a_{2 N+1}>0$. Define

$$
\begin{aligned}
& \mathrm{f}:[0,+\infty) \rightarrow \mathbb{R} ; t \mapsto e^{h(t)} \\
& g:[0,+\infty) \rightarrow \mathbb{R} ; t \mapsto t \mathrm{f}(t) ; \\
& G:[0,+\infty) \rightarrow \mathbb{R} ; t \mapsto \int_{0}^{t} g d \tau
\end{aligned}
$$

Then $G:[0,+\infty) \rightarrow[0,+\infty)$ is a bijection with inverse $G^{-1}$. Define the strictly increasing function

$$
J:[0,+\infty) \rightarrow \mathbb{R} ; t \mapsto g \circ G^{-1}
$$

Put $t_{h}:=G\left(a_{h}\right)$ for $h=0, \ldots, 2 N+1$. Then $+\infty>t_{0}>t_{1}>\cdots>t_{2 N}>$ $t_{2 N+1}>>0$. Put $B:=B(0, r)$ where $r:=G^{-1}(v / 2 \pi)$ so that $V_{f}(B)=v$. Note that 


$$
\begin{aligned}
v & =V_{f}(E)=2 \pi \sum_{h=0}^{N}\left\{G\left(a_{2 h}\right)-G\left(a_{2 h+1}\right)\right\} \\
& =2 \pi \sum_{h=0}^{2 N+1}(-1)^{h} t_{h} .
\end{aligned}
$$

By Lemma 10.5,

$$
\begin{aligned}
P_{f}(E) & =2 \pi \sum_{h=0}^{2 N+1} g\left(a_{h}\right)=2 \pi \sum_{h=0}^{2 N+1} J\left(t_{h}\right) \\
& \geq 2 \pi J\left(\sum_{h=0}^{2 N+1}(-1)^{h} t_{h}\right) \\
& =2 \pi J(v / 2 \pi)=P_{f}(B) .
\end{aligned}
$$

Proof of Theorem 1.2 Let $v>0$ and $E$ be a minimiser for (1.2). Then $E$ is essentially bounded by Theorem 3.1. By Theorem 4.5 there exists an $\mathscr{L}^{2}$-measurable set $\widetilde{E}$ with the properties

(a) $\widetilde{E}$ is a minimiser of (1.2);

(b) $L_{\widetilde{E}}=L_{E}$ a.e. on $(0,+\infty)$;

(c) $\widetilde{E}$ is open, bounded and has $C^{1,1}$ boundary;

(d) $\widetilde{E} \backslash\{0\}=\widetilde{E}^{s c}$.

(i) Suppose that $0<v \leq v_{0}$ so that $R>0$. Choose $r \in(0, R]$ such that $V(B(0, r))=$ $V(E)=v$. Suppose that $\widetilde{E} \backslash \bar{B}(0, R) \neq \emptyset$. By Lemma 10.4 there exists $t>R$ such that $\mathbb{S}_{t}^{1} \subset M$. As $g$ is strictly increasing, $g(t)>g(r)$. So $P_{f}(E)=P_{f}(\widetilde{E}) \geq \pi g(t)>$ $\pi g(r)=P_{f}(B(0, r))$. This contradicts the fact that $E$ is a minimiser for (1.2). So $\widetilde{E} \subset \bar{B}(0, R)$ and $L_{\widetilde{E}}=0$ on $(R,+\infty)$. By property $(\mathrm{b}),|E \backslash \bar{B}(0, R)|=0$. By the uniqueness property in the classical isoperimetric theorem (see for example [12] Theorem 4.11) the set $E$ is equivalent to a ball $B$ in $\bar{B}(0, R)$.

(ii) With $r>0$ as before, $V(B(0, r))=V(E)=v>v_{0}=V(B(0, R))$ so $r>R$. If $\widetilde{E} \backslash \bar{B}(0, r) \neq \emptyset$ we derive a contradiction in the same way as above. Consequently, $\widetilde{E}=B:=B(0, r)$. Thus, $L_{E}=L_{B}$ a.e. on $(0,+\infty)$; in particular, $|E \backslash B|=0$. This entails that $E$ is equivalent to $B$.

Open Access This article is distributed under the terms of the Creative Commons Attribution 4.0 International License (http://creativecommons.org/licenses/by/4.0/), which permits unrestricted use, distribution, and reproduction in any medium, provided you give appropriate credit to the original author(s) and the source, provide a link to the Creative Commons license, and indicate if changes were made. 


\section{References}

1. Ambrosio, L., Fusco, N., Pallara, D.: Functions of Bounded Variation and Free Discontinuity Problems. Oxford University Press, Oxford (2000)

2. Barchiesi, M., Cagnetti, F., Fusco, N.: Stability of the Steiner symmetrization of convex sets. J. Eur. Math. Soc. 15, 1245-1278 (2013)

3. Borell, C.: The Ornstein-Uhlenbeck Velocity Process in Backward Time and Isoperimetry. Chalmers Tekniska Hógskola, Göteborgs Universitet, Gothenburg (1986)

4. Boyer, W., Brown, B., Chambers, G., Loving, A., Tammen, S.: Isoperimetric regions in $\mathbb{R}^{n}$ with density $r^{p}$, Analysis and Geometry in Metric Spaces, ISSN (Online) pp. 2299-3274 (2016)

5. Betta, F.M., Brock, F., Mercaldo, A., Posteraro, M.R.: Weighted isoperimetric inequalities on $\mathbb{R}^{n}$ and applications to rearrangements. Math. Nachr. 281(4), 466-498 (2008)

6. Carothers, N.L.: Real Analysis. Cambridge University Press, Cambridge (2000)

7. Chambers, G.R.: Proof of the log-convex density conjecture. To appear in J. Eur. Math. Soc (2016)

8. Cinti, E., Pratelli, A.: Regularity of isoperimetric sets in $\mathbb{R}^{2}$ with density. To appear in Math. Ann. (2017)

9. De Giorgi, E.: Sulla proprietá isoperimetrica dell'ipersfera, nella classe degli insiemi aventi frontiera orientata di misura finita. Atti Accad. Naz. Lincei Mem. Cl. Sci. Fis. Mat. Mat. Sez. I 5, 33-44 (1958)

10. Dubins, L.E.: On curves of minimal length with a constraint on average curvature, and with prescribed initial and terminal positions and tangents. Am. J. Math. 79(3), 497-516 (1957)

11. Figalli, A., Maggi, F.: On the isoperimetric problem for radial log-convex densities. Calc. Var. Partial Differ. Equ. 48(3-4), 447-489 (2013)

12. Fusco, N.: The classical isoperimetric theorem. Rend. Acc. Sci. Fis. Mat. Napoli 4(71), 63-107 (2004)

13. Giusti, E.: Minimal Surfaces and Functions of Bounded Variation. Birkhäuser, Boston (1984)

14. Gradshteyn, I.S., Ryzhik, I.M.: Tables of Integrals, Series and Products. Academic Press, New York (1965)

15. Hadamard, J.: Étude sur les propriétés des fonctions entieres et en particulier d'une fonction considérée par Riemann. J. Math. Pures et Appl. 58, 171-215 (1893)

16. Hale, J.: Ordinary Differential Equations. Wiley, Hoboken (1969)

17. Hermite, C.: Sur deux limites d'une intégrale définie. Mathesis 3, 82 (1883)

18. Howard, H., Treibergs, A.: A reverse isoperimetric inequality, stability and extremal theorems for plane curves with bounded curvature. Rocky Mt. J. Math. 25(2), 635-684 (1995)

19. Kolesnikov, A.V., Zhdanov, R.I.: On Isoperimetric Sets of Radially Symmetric Measures, Concentration, Functional Inequalities and Isoperimetry, Contemporary Mathematics, vol. 545, pp. 123-154. American Mathematical Society, Providence (2011)

20. Lee, J.M.: Manifolds and Differential Geometry. American Mathematical Society, Providence (2009)

21. Morgan, F.: Regularity of isoperimetric hypersurfaces in Riemannian manifolds. Trans. AMS 355(12), 5041-5052 (2003)

22. Morgan, F., Pratelli, A.: Existence of isoperimetric regions in $\mathbb{R}^{n}$ with density. Ann. Glob. Anal. Geom. 43(4), 331-365 (2013)

23. Perugini, M.: Ph.D. Thesis (in preparation), University of Sussex (2016)

24. Rosales, C., Cañete, A., Bale, V., Morgan, F.: On the isoperimetric problem in Euclidean space with density. Calc. Var. Partial Differ. Equ. 31(1), 27-46 (2008)

25. Simmons, G.F.: Introduction to Topology and Modern Analysis. McGraw-Hill, New York (1963)

26. Spivak, M.: A Comprehensive Introduction to Differential Geometry, Publish or Perish, vol. 2 (1970)

27. Tamanini, I.: Regularity results for almost minimal oriented hypersurfaces in $\mathbb{R}^{n}$, Quaderni del Dipartimento di Matematica dell' Universita di Lecce (1984) 